\title{
Central and Peripheral Metabolic Defects Contribute to the Pathogenesis of Alzheimer's Disease: Targeting Mitochondria for Diagnosis and Prevention
}

Yunhua Peng, Peipei Gao, Le Shi, Lei Chen, Jiankang Liu, and Jiangang Long

\begin{abstract}
Significance: Epidemiological studies indicate that metabolic disorders are associated with an increased risk for Alzheimer's disease (AD). Metabolic remodeling occurs in the central nervous system (CNS) and periphery, even in the early stages of AD. Mitochondrial dysfunction has been widely accepted as a molecular mechanism underlying metabolic disorders. Therefore, focusing on early metabolic changes, especially from the perspective of mitochondria, could be of interest for early AD diagnosis and intervention.

Recent Advances: We and others have identified that the levels of several metabolites are fluctuated in the periphery before their accumulation in the CNS, which plays an important role in the pathogenesis of AD. Mitochondrial remodeling is likely one of the earliest signs of $\mathrm{AD}$, linking nutritional imbalance to cognitive deficits. Notably, by improving mitochondrial function, mitochondrial nutrients efficiently rescue cellular metabolic dysfunction in the CNS and periphery in individuals with AD.

Critical Issues: Peripheral metabolic disorders should be intensively explored and evaluated for the early diagnosis of $\mathrm{AD}$. The circulating metabolites derived from mitochondrial remodeling represent novel potential diagnostic biomarkers for AD that are more readily detected than CNS-oriented biomarkers. Moreover, mitochondrial nutrients provide a promising approach to preventing and delaying AD progression.

Future Directions: Abnormal mitochondrial metabolism in the CNS and periphery is involved in AD pathogenesis. More clinical studies provide evidence for the suitability and reliability of circulating metabolites and cytokines for the early diagnosis of $\mathrm{AD}$. Targeting mitochondria to rewire cellular metabolism is a promising approach to preventing AD and ameliorating AD-related metabolic disorders. Antioxid. Redox Signal. 32, 1188-1236.
\end{abstract}

Keywords: glucose, ketogenesis, lipogenesis, mitochondrial nutrients, biomarkers

\section{Table of Contents}

I. Introduction

A. A $\beta$ and amyloid plaques 1189

1. $\mathrm{A} \beta$ and mitochondria

1191

B. Hyperphosphorylation of the Tau protein and NFTs 1191

C. Oxidative stress and inflammation 1192

D. Mitochondrial dysfunction 1192

II. Glucose Metabolism in AD 1193

$\begin{array}{ll}\text { A. Glucose uptake deficit } & 1193 \\ \text { B. Insulin resistance } & 1194 \\ \text { C. Glycosylation } & 1195\end{array}$

Reviewing Editors: Jie Bai, Enrique Cadenas, Vittorio Calabrese, Myra Conway, Isidre Ferrer, Reddy Peera Kommaddi, Paula Moreira, Antonio Orlacchio, George Perry, Ana Cristina Rego, and Rommy von Bernhardi

Center for Mitochondrial Biology \& Medicine, The Key Laboratory of Biomedical Information Engineering of Ministry of Education, School of Life Science and Technology, Frontier Institute of Science and Technology, Xi' an Jiaotong University, Xi'an, China. 
III. Lipid Metabolism in AD

A. Lipid dysregulation as an early signal in AD 1196

B. Lipogenesis in AD $\quad 1198$

$\begin{array}{ll}\text { C. Lipolysis in AD } & 1198\end{array}$

D. Ketogenesis in AD $\quad 1198$

IV. Mitochondria at the Metabolic Core in AD 1199

A. Mitochondrial dysfunction precedes metabolic disorder in AD 1199

B. Mitochondria mediate pathological impairment during AD progression $\quad 1199$

C. Mitochondrial remodeling in AD 1200

D. Mitochondria-induced inflammation and oxidative stress during the pathogenesis of AD 1200

E. Mitochondrial metabolic disorder links nutritional imbalance to neural DNA epigenetic remodeling $\quad 1202$

V. Mitochondria-Centered Metabolic Markers in Early AD 1202

A. Biomarkers in the brain and CSF 1203

B. Biomarkers in peripheral tissues and circulating body fluids $\quad 1204$

VI. Targeting Mitochondrial Metabolic Dysfunction for AD Prevention 1204

A. Mitochondrial nutrients $\quad 1205$

$\begin{array}{ll}\text { 1. Lipoic acid } & 1205\end{array}$

2. Acetyl-L-carnitine 1206

3. Coenzyme $\mathrm{Q}_{10}$ and MitoQ 1206

4. B vitamins 1209

5. Hydroxytyrosol $\quad 1209$

6. $\beta$-hydroxybutyrate $\quad 1210$

7. Hydrogen 1211

8. Other mitochondrial nutrients for AD 1211

9. Combination of mitochondrial nutrients 1212

B. Exercise 1212

1. Exercise improves the metabolic profile in AD 1212

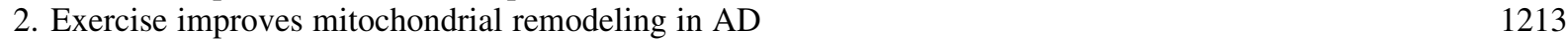

3. Exercise decreases inflammation in AD 1213

C. Natural compounds from traditional Chinese medicines 1213

1. TCMs ameliorate insulin resistance and hepatic metabolic dysfunction $\quad 1213$

2. TCMs ameliorate mitochondrial dysfunction and oxidative stress 1214

3. TCMs ameliorate inflammation 1215

4. A variety of TCMs with mitochondria-related functional readouts may reveal a new therapeutic strategy in AD treatment $\quad 1215$

\begin{tabular}{lr} 
VII. Concluding Remarks & 1217 \\
\hline
\end{tabular}

\section{Introduction}

A LZHEIMER'S DISEASE (AD), THE most common type of dementia, is a progressive and irreversible neurodegenerative disease, characterized by a progressive loss of learning, memory, orientation, language, comprehension, judgment, and intellectual performance $(8,430)$. This disease was first observed in 1906 by Dr. Alois Alzheimer, who found abnormal plaques and fibrillary pathology in the brain of his patient Auguste Deter, who died due to an atypical mental illness (7). In the United States, $\sim 5.8$ million patients are living with $\mathrm{AD}$, the majority of whom are older than 65 years. Statistics show that in 2010, about 10 million people suffered from AD in China, which is the country with the largest number of patients in the world. The prevalence of AD in China was estimated at $3.21 \%$ among people aged $\geq 65$ years (181). AD is the sixth leading cause of death in the United States: Deaths from AD have increased $\sim 1.5$-fold, whereas deaths from others have decreased during the past two decades.

Once clinically diagnosed, the patient has an average life expectancy of 3 to 9 years. In the clinic, only a few Food and Drug Administration drugs have been approved for the treatment of $\mathrm{AD}$, including donepezil, rivastigmine, galantamine, and memantine; however, these drugs have limited abilities to reverse AD progression and only temporarily improve the quality of life for patients $(8,158)$.

Pathologically, $\mathrm{AD}$ is characterized by $\mathrm{A} \beta$ and neurofibrillary tangles (NFTs) in the brain. Although there is no conclusion about the molecular mechanism(s) of AD, possibilities include (i) beta amyloid $(\mathrm{A} \beta)$ and amyloid plaques, (ii) hyperphosphorylation of the Tau protein and NFTs, (iii) oxidative stress and inflammation, (iv) and mitochondrial dysfunction (Fig. 1).

\section{A. $A \beta$ and amyloid plaques}

$\mathrm{AD}$ can be divided into three main types: early-onset familial AD (FAD), early-onset sporadic AD, and late-onset sporadic AD. The early-onset FAD subtype accounts for $\sim 1 \%$ of all AD cases, with 326 total families worldwide living with this type of AD. The genetic variants in autosomal dominant FAD include genes encoding the transmembrane protein amyloid beta precursor protein (APP), presenilin 1 (PSEN1, also known as PS1), and presenilin 2 (PSEN2) (311, $336,394)$, which are all involved in APP processing. 


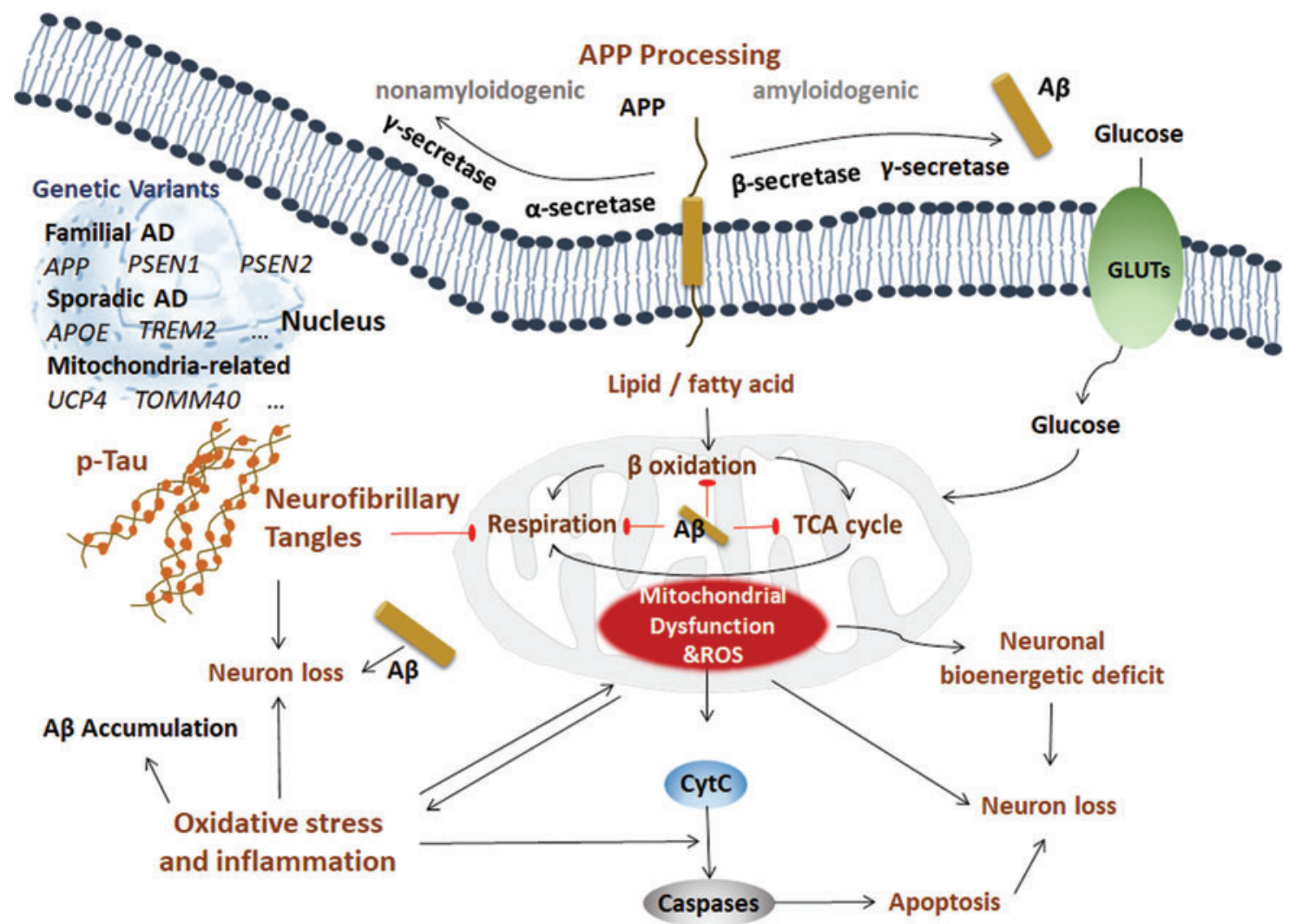

FIG. 1. Possible mechanisms of AD. Pathologically, AD is characterized by $\mathrm{A} \beta$ and NFTs in the brain. However, the molecular mechanism(s) of AD is inconclusive. The possibilities include (i) A $\beta$ and amyloid plaque. APP is a transmembrane protein that can be processed in two distinct ways: nonamyloidogenic processing and amyloidogenic processing. In the nonamyloidogenic processing method, APP is sequentially cleaved by $\alpha$-secretase and $\gamma$-secretase. In amyloidogenic processing, APP is sequentially cleaved by $\beta$-secretase and $\gamma$-secretase, producing toxic A $\beta$ fragments, which play roles in both CNS and periphery. This sequential cleavage occurs on the plasma membranes of neurons, adipocytes, and hepatocytes. The genetic variants in autosomal dominant FAD include genes encoding the transmembrane protein APP, PSEN1, and PSEN2. The most common variant in sporadic AD patients is APOE. The GWAS identified other rare genetic variants such as TREM2. The mitochondria-related variants such as $U C P 2$ and TOMM4O are also identified. (ii) Hyperphosphorylation of Tau protein and NFTs. Tau is the primary component of NFTs in AD, and the most prominent post-translational modification of Tau in AD is hyperphosphorylation. Hyperphosphorylated Tau eventually forms abundant NFTs, which is toxic to synapses and neurons and impairs cognitive function. (iii) Oxidative stress and inflammation. Mitochondriaassociated oxidative damage and the inflammatory response are early key factors in the development of $\mathrm{AD}$. A $\beta$ directly dampens mitochondrial structure and function, which promotes oxidative stress and inflammation, and further facilitates the pathogenesis of AD. (iv) Mitochondrial dysfunction. Two major biological functions that occur in mitochondria are the TCA cycle and respiration. Hyperglycemia and hyperlipidemia impair mitochondrial function and disturb the mitochondrial homeostasis, leading to metabolic disorder and neuronal bioenergetic deficit in AD. A $\beta$, beta amyloid; AD, Alzheimer's disease; $A P O E$, apolipoprotein E; APP, amyloid beta precursor protein; CNS, central nervous system; FAD, familial AD; GLUTs, glucose transporters; GWAS, genome-wide association studies; NFTs, neurofibrillary tangles; PSEN1, presenilin 1; PSEN2, presenilin 2; TCA, tricarboxylic acid; TOMM40, translocase of outer mitochondrial membrane 40; TREM2, triggering receptor expressed on myeloid cells 2; UCP, mitochondrial uncoupling proteins. Color images are available online.

The APP gene is located on chromosome 21, and the mutations include A201V (344), A235V (295), D243N (295), E246K (111), E296K (295), P299L (295), R468H (347), A479S (111), K496Q (344), A500T (347), Y538H (285, 344), V562I (344), E599K (344), T600M (347), P620A (295), P620L (344), T663M (347), E665D (306), V669L (Seoul) (20), KM670/671NL (283), A673T (306), A673V (79), H677R (179), D678H (52), D678N (403), E682K (472), K687N (187), A692G (154), E693G (188), E693K (383), E693Q (221), D694N (128), L705V (297), G708G (21), G709S (347), A713T (47), T714A (304), T714I (210), V715A (70), V715M (11), I716F (139), I716M (32), I716T
(391), I716V (94), V717F (284), V717G (51), V717I (125), V717L (285), T719N (168), T719P (121), M722K (416), L723P (211), and K724N (392).

PSEN1 and PSEN2 are located on chromosomes 14 and 1 and contain 13 exons and 12 exons, respectively, and more than 300 mutations have been reported in PSEN, which represents the most common genetic variation in AD (214). On the other hand, PSEN2 is very rarely mutated and is related to $\mathrm{AD}$ (44). Mutations in the $A P P$ gene trigger the overproduction of neurotoxic and aggregation-prone forms of A $\beta$ peptides by shifting the cleavage of APP toward amyloidogenic processing (24). The PSEN1 and PSEN2 genes 
aggravate the production ratio of $\mathrm{A} \beta_{42}$ by regulating the $\gamma$ secretase-mediated cleavage of APP (24). However, the majority of sporadic AD cases have no such mutations in $A P P$, PSEN, and PSEN2 (186), indicating a different or additional mechanism underlying AD pathogenesis.

APP is a transmembrane protein that can be processed in two distinct ways: nonamyloidogenic processing and amyloidogenic processing. In the nonamyloidogenic processing method, APP is sequentially cleaved by $\alpha$-secretase and $\gamma$-secretase (PS1) into a secreted C-terminal fragment (sAP$\mathrm{P} \alpha$ ), p3 and amyloid intracellular domain. In amyloidogenic processing, APP is sequentially cleaved by $\beta$-secretase ( $\beta$-site APP cleaving enzyme 1 [BACE1]) and $\gamma$-secretase, producing toxic $\mathrm{A} \beta$ fragments. This sequential cleavage occurs on the plasma membranes of neurons, adipocytes, and hepatocytes $(463,473)$. A $\beta$ deposition in the central nervous system (CNS) serves as the most significant pathological hallmark of $\mathrm{AD}$ at patient autopsy (8). Under pathological conditions, amyloidogenic processing predominates, and AD is thus initiated by an imbalance between the formation and degradation of $\mathrm{A} \beta$, leading to the deposition of $\mathrm{A} \beta$ and subsequent disruption of synapse and neuronal function (423), representing the so-called $\mathrm{A} \beta$ hypothesis (151). Moreover, $\mathrm{A} \beta$ has expanded roles in other peripheral organs (412).

The $\mathrm{A} \beta$ peptide constitutes 37 to 43 amino acids, most of which are $\mathrm{A} \beta_{40}$ and $\mathrm{A} \beta_{42}$. $\mathrm{A} \beta_{42}$ differs from $\mathrm{A} \beta_{40}$ by two extra isoleucine and alanine residues at the C-terminus. $\mathrm{A} \beta_{42}$ is the major component of plaque deposition, whereas $\mathrm{A} \beta_{40}$ is the major component of cerebrovascular $\mathrm{A} \beta$ (137). $\mathrm{A} \beta_{42}$ is more amyloidogenic and has a higher cellular toxicity than $\mathrm{A} \beta_{40}$ (319). A higher $\mathrm{A} \beta_{40} / \mathrm{A} \beta_{42}$ ratio may promote the construction of cerebral amyloid angiopathy over parenchymal deposition $(156,443)$. The $\mathrm{A} \beta$ peptide can be cleared under normal conditions but accumulates as neurotoxic $\mathrm{A} \beta$ derived diffusible ligands in $\mathrm{AD}$ pathogenesis. $\mathrm{A} \beta$ peptide deposition by the self-assembly and aggregation of $4 \mathrm{kD} \mathrm{A} \beta$ peptides not only shows strong neurotoxicity related to neuronal degeneration but also induces a series of pathological events, such as astrocyte and microglia activation, blood-brain barrier (BBB) destruction, and microcirculation changes, leading to neurodegeneration and death in AD (349). $\mathrm{A} \beta$ can be degraded by insulin degrading enzyme (IDE), neprilysin (NEP), and other degrading enzymes (339).

The most common variant in sporadic $\mathrm{AD}$ patients is apolipoprotein $\mathrm{E}(A P O E)$, which not only increases the severity of FAD but also increases the susceptibility to sporadic $\mathrm{AD}$ (172). ApoE is a lipid-binding protein that modulates triglyceride and cholesterol transportation in the liver, brain, and other tissues. The $A P O E$ type 4 allele (APOE4) is the main genetic risk factor for sporadic AD (64), whereas the APOE2 genotype has been identified as a protective factor for late-onset sporadic AD (63). Mechanically, ApoE has an $\mathrm{A} \beta$-binding motif and serves as a clearance protein to induce toxic $\mathrm{A} \beta$ degradation in lysosomes.

According to genome-wide association studies, other rare genetic variants accounting for late-onset $\mathrm{AD}$ have been identified, including genes encoding triggering receptor expressed on myeloid cells 2 (TREM2), complement C3b/C4b receptor 1 (CR1), bridging integrator 1 (BIN1), phosphatidylinositol binding clathrin assembly protein (PICALM), clusterin (CLU), adenosine triphosphate (ATP) binding cassette subfamily A member 7 (ABCA7), and CD33 molecule
(CD33) $(113,138,152,160,213)$. These genetic variants are all related to $\mathrm{A} \beta$ deposition; for example, TREM 2 and CR1 play a key role in the removal of cell debris and $\mathrm{A} \beta$ clearance (138).

Although preclinic and clinical studies show that $\mathrm{A} \beta$ pathology is the major characteristic of $\mathrm{AD}$, many clinical trials focusing on clearing $\mathrm{A} \beta$ and decreasing $\mathrm{A} \beta$ production have failed $(8,162,209,234,405)$, indicating that track- $A \beta$ only is insufficient for $\mathrm{AD}$ treatment and that additional mechanisms are essential for the pathogenesis of AD.

Notably, some genetic variants of AD are involved in not only classic $\mathrm{A} \beta$ pathology but also cellular metabolism (e.g., $A B C A 7, A P O E 4, C L U)$ and inflammation (e.g., CLU, CD33). Although there is no consistent evidence of mitochondrial DNA (mtDNA) variants in AD $(171,312)$, mitochondriarelated variants were identified in $\mathrm{AD}$. For example, the genetic variant in mitochondrial uncoupling protein 4 (UCP4), which encodes the mitochondrial inner membrane transporter uncoupling protein and is responsible for mitochondrial energy metabolism, markedly affects the susceptibility to $\mathrm{AD}$ (274). Variants in intron 6 of translocase of outer mitochondrial membrane 40 (TOMM40), which encodes the central pore of the translocase of the mitochondrial outer membrane and controls protein entry into the mitochondria, are associated with the risk of $\mathrm{AD}$ (456). With the advanced technologies for genetics and omics studies, an increasing number of genetic variants can be identified to uncover the mechanisms and new treatments for AD.

1. $\mathrm{A} \beta$ and mitochondria. Several studies have suggested that $\mathrm{A} \beta$ can directly affect mitochondrial function $(6,10,36$, $84,248,303)$. The amino terminus of APP enters mitochondria depending on the mitochondrial outer membrane translocase TOM40 and inner membrane translocases TIM23 and TIM44, whereas the carboxy terminus of APP cannot, resulting in blockage of the mitochondrial protein import channel (10). This blockage hinders other proteins from entering the mitochondria, causing decreased cytochrome oxidase activity, increased production of reactive oxygen species (ROS), and mitochondrial dysfunction (10). A $\beta$ itself can bind to alcohol dehydrogenase $(\mathrm{ADH})$ in mitochondria to form $\mathrm{A} \beta$-binding alcohol dehydrogenase (ABAD), promote ROS production, and damage mitochondrial function (248). $\mathrm{A} \beta$ also regulates $\mathrm{Ca}^{2+}$ homeostasis, leading to opening of the mitochondrial permeability transition pore (mPTP) to decrease the mitochondrial membrane potential, matrix swelling, and respiratory damage, which can be reversed by several compounds, such as the mitochondrial osmotic transfer channel inhibitor cyclosporine A (CsA), adenosine diphosphate (ADP), and oligomycin. Notably, ADP together with oligomycin works better in brain mitochondria than CsA $(6,36,303)$. Mitochondria are the core producers of ROS in the cell, and $\mathrm{A} \beta$ is partially targeted to mitochondria to promote ROS and mitochondrial dysfunction $(84,248)$.

\section{B. Hyperphosphorylation of the Tau protein and NFTs}

Another significant pathological hallmark of AD is NFTs, which indicates the severity of AD $(134,177)$. Although NFTs exist in many diseases, such as AD, ischemia, and stroke, their pathology is distinct among each disease. Tau is the primary component of NFTs in AD and contains 
microtubule-binding domains, projection domains, and prolinerich regions with multiple phosphorylation sites $(29,217)$.

The Tau protein is an unfolded and soluble protein that assembles and stabilizes microtubules to maintain neuronal function. In the brains of AD patients, Tau undergoes multiple post-translational modifications, such as hyperphosphorylation and glycosylation $(134,413)$. The most prominent post-translational modification of Tau in AD is hyperphosphorylation. Thus far, numerous phosphorylation sites have been identified, including Ser199, Ser202, Ser262, Ser422, Ser396, Thr205, and Thr231 (18, 351). Several kinases, including cyclin-dependent kinase 5 (CDK5), mitogenactivated protein kinase (MAPK), and GSK-3 $\beta$, have been demonstrated to regulate Tau phosphorylation $(62,277)$. Hyperphosphorylated Tau forms Tau dimers, which then form Tau oligomers. The aggregated and misfolded Tau oligomers form paired helical filaments, which eventually form abundant NFTs. The hyperphosphorylated Tau is toxic to synapses and neurons and impairs cognitive function (62). The N-glycosylation of Tau inhibits its binding with microtubules (413). Another type of glycosylation, O-GlcNAcylation, is negatively correlated with Tau phosphorylation, indicating an effect opposite that of $\mathrm{N}$-glycosylation (230).

A $\beta$ also promotes Tau accumulation. Crossing transgenic APP mice with Tau mice induces neurotoxicity, indicating that tauopathy is also required for $\mathrm{A} \beta$ toxicity (334). In addition, the $\mathrm{A} \beta_{42}$ oligomer induces progressive tauopathy in neurons (369). The prolyl isomerase Pin1 is downregulated by oxidation in AD. The deficiency of Pin 1 promotes glycogen synthesis kinase 3 (GSK3) to hyperphosphorylate Tau, leading to tangle formation and neuronal cell death (305). On the other hand, Pin1 also binds to the p-Thr668 motif in APP to increase $\mathrm{A} \beta$ production (305). Apart from $\mathrm{A} \beta$, tauopathy may lead to cognitive dysfunction through gray matter loss in AD (27). Although the mechanistic relationship between mitochondria and tauopathy is elusive, the hyperphosphorylation of Tau is known to be associated with altered mitochondrial distribution, increased abnormal mitochondrial trafficking, and impaired mitochondrial dynamics and functions $(190,204,260)$.

\section{Oxidative stress and inflammation}

Systemic inflammatory responses and oxidative stress have been recognized as two other moderators and biomarkers of AD. Oxidative stress and inflammation are largely attributed to mitochondrial dysfunction in the brain and periphery, leading to energy metabolism failure and neurological disorders (321). Oxidative stress and inflammation induced by mitochondrial damage occur before AD pathological features, such as $\mathrm{A} \beta$ deposition, and accelerate AD progression (451). Although inflammation, oxidative stress, and related mitochondrial dysfunction are not specific to $\mathrm{AD}$, they overlap extensively with $\mathrm{AD}$ pathogenesis and contribute to $\mathrm{AD}$ progression. On the one hand, mitochondria-associated oxidative damage and the inflammatory response are early key factors in the development of $\mathrm{AD}$. On the other hand, $\mathrm{A} \beta$, as the most typical pathological feature of $\mathrm{AD}$, directly dampens mitochondrial structure and function, which promotes oxidative stress and inflammation in neurons, and further facilitates the pathogenesis of $\mathrm{AD}$.

\section{Mitochondrial dysfunction}

The "Mitochondrial cascade hypothesis" and "mitochondrial bottleneck hypothesis" suggest that mitochondrial dysfunction is an essential early and primary event in AD pathogenesis $(73,378,380)$. Swerdlow and Khan proposed the mitochondrial cascade hypothesis to show that mitochondrial dysfunction is a primary event that causes all AD pathogenic changes, including $\mathrm{A} \beta$ deposition, NFT formation, neuron death, and synaptic loss (379-381). In early research, several groups observed different types of mitochondrial abnormalities in the brains or peripheral tissues of patients with AD and animal models (22, 157, 248, 270, 354, 419). Using genetic AD animal models, many studies have shown that mitochondrial bioenergetic deficits occur in these models at very early ages, and mitochondrial dysfunction precedes Alzheimer's pathology $(85,448)$. Studies by us and others have shown that targeting mitochondria to improve mitochondrial biogenesis, remodeling mitochondrial fusion/ fission, or promoting mitochondrial respiration function with pharmaceutics and nutrient approaches efficiently slow down or reverse the pathogenesis of $\operatorname{AD}(233,234,356)$. Taken together, these studies indicate that mitochondrial dysfunction plays a key role in the pathogenesis of AD.

Two major biological functions that occur in mitochondria are the tricarboxylic acid cycle (TCA cycle, also known as the Krebs cycle) and oxidative phosphorylation (OXPHOS) (266). Although the three major nutrients, glucose, lipids, and proteins, have different metabolic pathways in vivo, they all share the common intermediate metabolite acetyl-CoA, which is further utilized by the TCA cycle to produce NADH (313). NADH is further used as a substrate for OXPHOS to produce ATP. The high-order assembly of respiratory chain complexes in the inner mitochondrial membrane allows mitochondria to respond to energy conversion to promote efficient electron transfer (141). The process of electron transfer in the respiratory chain is coupled to ADP phosphorylation to generate ATP. The ATP-synthase activity is also significantly lower in AD patients in the stage that is characterized by entorhinal and transentorhinal tauopathy with no clinical symptoms than in age-matched controls. Targeted for oxidative stress, the mitochondrial ATP-synthase $\alpha$ subunit is lipoxidized distinctly in the entorhinal cortex in the AD cases. This study specifies that the energy production system in mitochondria may be one pathway that is impaired in the very early stages of AD (390). A reduced cytochrome c oxidase $(\mathrm{COX})$ subunit 4 in mitochondrial electron transport chain links amyloid deposition and mitochondria dysfunction in the dystrophic neurites of senile plaques. However, a preserved COX subunit 4 in neurons is associated with NFTs (310).

Epidemiological studies indicate that hyperglycemia and hyperlipidemia are major risk factors for neurodegenerative diseases, especially AD (69, 201, 226). The 2019 World Health Organization (WHO) guidelines for the reduction of cognitive decline and dementia risks recommended the management of diabetes in adults and the management of dyslipidemia at midlife to reduce the risk of cognitive decline and/or dementia (425). Indeed, we and others have recently shown that deficits in glucose and lipid metabolism occur early and before significant cognitive decline (412).

Apart from their key role in bioenergy metabolism, mitochondria represent the "integration" center of signal 
transduction and regulate epigenetics, stem cells, and the differentiation, initiation, and execution of apoptosis. Mitochondria are capable of sensing cellular stress and helping cells adapt to challenging microenvironment conditions, giving cells a high degree of plasticity to promote their growth and survival. These abilities suggest that mitochondria are uniquely significant in age-related diseases, especially in AD progression (378).

Each suggested hypothesis functions in the AD pathogenesis feedback loop, and they coparticipate with each other at multiple levels during $\mathrm{AD}$ progression. Further, mitochondria serve as the bridge among glucose metabolism, lipid metabolism, oxidative stress, inflammation, and AD pathogenesis. Mitochondrial dysfunction and mitochondrial remodeling may provoke $\mathrm{AD}$ pathogenesis and lead to $\mathrm{AD}$ progression. A potential role of mitochondria at the core of metabolism during AD progression deserves more attention (Fig. 2).

\section{Glucose Metabolism in AD}

Glucose is the only energy source that can cross the BBB, and it provides energy for normal neuronal functions. $\mathrm{Nu}-$ merous studies have identified impairments in glucose utilization and metabolism in the brain and peripheral tissues of AD patients and animal models. Alterations in glucose metabolism occur with the accumulation of oxidative damage before $\mathrm{AD}$ pathology in the brains of $\mathrm{AD}$ patients and animal models $(89,227,272,279)$.

\section{A. Glucose uptake deficit}

Glucose cannot be synthesized or stored in the brain. Dr. Magistretti discovered that glycogen in the brain is enriched in astrocytes, indicating that glucose from the blood first enters and is stored in astrocytes $(41,253)$. This finding has led to the initial discovery of glucose transport, storage, and transformation pathways in the brain. Thus, subsequent series of studies showed that when neurons are activated, they release glutamate signals, stimulate astrocytes to break down glycogen, and convert glucose into pyruvate and, finally, lactic acid, which is released extracellularly by a monocarboxylic acid transporter and taken up by neighboring neurons to produce ATP (254).

Glucose uptake is markedly reduced in early-stage AD patients, suggesting that decreased glucose metabolism and

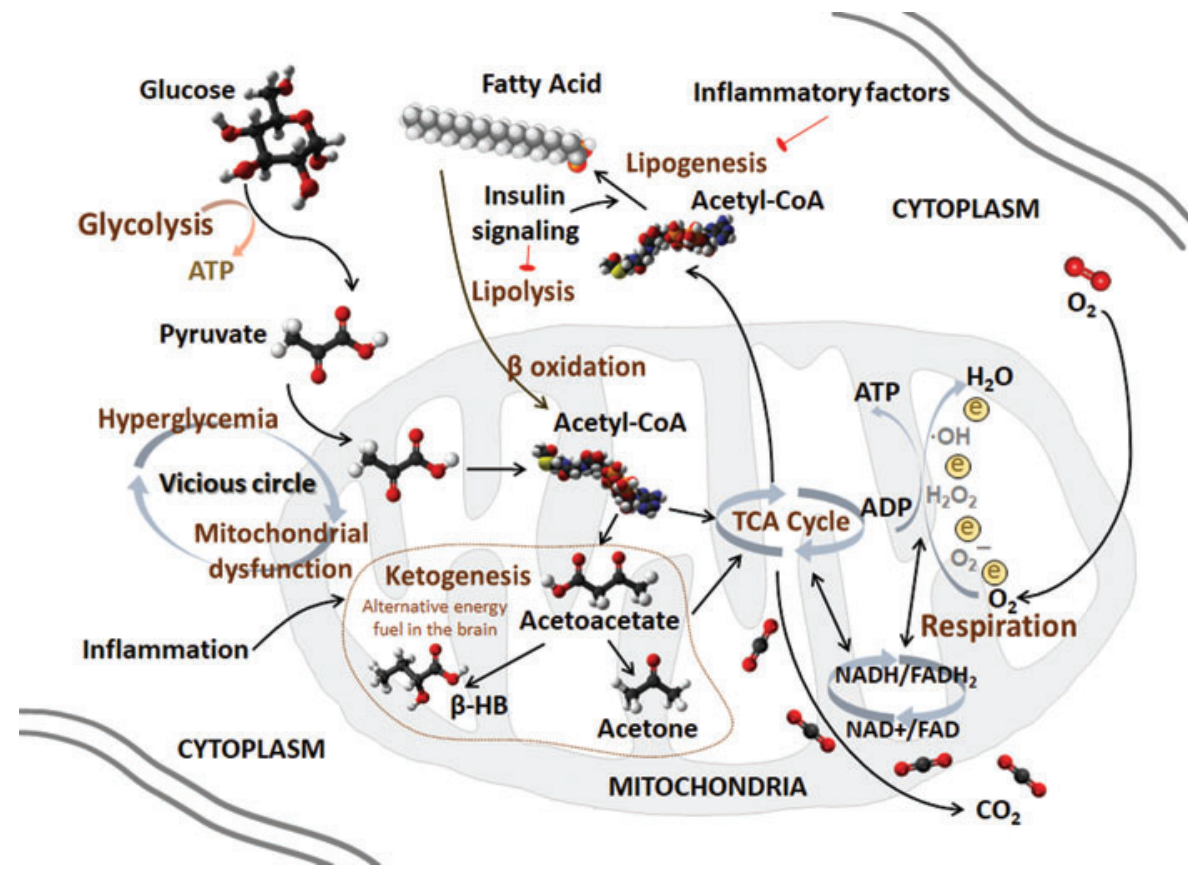

FIG. 2. Mitochondria act as the key organelle for glucose and lipid metabolism. Mitochondria are energy generators of cells with two biological functions, the TCA cycle and respiration. The TCA cycle is a circulatory system for the enzymatic reaction of oxidizing acetyl-CoA to $\mathrm{CO}_{2}$ and is simultaneously coupled with OXPHOS to provide energy. The respiration chain is coupled to the production of ATP from ADP while transferring electrons to oxygen, which is reduced to $\mathrm{H}_{2} \mathrm{O}$ after the reaction. NADH and $\mathrm{FADH}_{2}$ are products of the TCA cycle and substrates for respiration. Glucose and lipids are the major energy substances in the periphery. The brain mainly uses glucose as the sole energy substrate under normal conditions. Glucose is decomposed to pyruvate to produce acetyl-CoA, which initiates the TCA cycle. A vicious cycle of hyperglycemia and mitochondrial dysfunction exists in the CNS and the periphery. Although lipids cannot be energy substrates in the brain, the adult brain is highly enriched in lipids, which not only constitute the structure of membrane system but also participate in signal transduction. Acetyl-CoA is also the subunit for fatty acid synthesis, which is an important process of lipogenesis. The lipogenic pathway can be inactivated by inflammatory factors, whereas insulin signaling stimulates lipogenesis and reduces lipolysis. Under energy-demanding conditions, lipolysis is triggered to release fatty acids and the mitochondrial beta-oxidation ensues for Acetyl-CoA generation, which is enrolled in ketogenesis stimulated by inflammatory factors in the early stage of AD. Ketone bodies, such as acetoacetate and $\beta$-HB, are used as neuronal alternative energy fuels. $\beta$ - $\mathrm{HB}, \beta$-hydroxybutyrate; ADP, adenosine diphosphate; ATP, adenosine triphosphate; OXPHOS, oxidative phosphorylation. Color images are available online. 
increased steady-state concentrations of glucose are early signs of $\mathrm{AD}(69,178,368,370)$. The age and sex-adjusted hazard ratios of $\mathrm{AD}$ in diabetic individuals were reported to be 1.94 times higher than those in the control group, indicating that diabetes itself is an independent risk factor for AD (298).

Using fluoro-2-deoxy-D-glucose positron emission tomography (FDG-PET) scanning, the cerebral metabolic rate of glucose utilization was demonstrated to progressively decline in patients with AD (280). Previous studies have shown that the cerebral metabolic rate of glucose utilization in the $\mathrm{AD}$ patient brain is $17 \%-24 \%$ lower than that in the normal brain (75). The decline in the cerebral glucose metabolic rate correlates with reduced cognitive scores and $\mathrm{AD}$ pathological processes. Moreover, individuals with declined cerebral metabolic rates of glucose utilization are at a higher risk for $\mathrm{AD}$ than those with normal rates (75).

The polar, hydrophilic glucose molecules require specific transporters to cross the cellular membrane. Glucose uptake functions via glucose transporters (GLUTs) at the BBB to transport glucose between the brain and periphery. The BBB is mainly formed by endothelial cells, astrocytes, and the basement membrane (195). Glucose transporters GLUT1 and GLUT3 are major GLUTs required for neuronal and glial glucose transport via the BBB $(401,402)$. GLUT1 is encoded by the Slc2a1 gene, and GLUT1 at the BBB mediates glucose transport to the brain. Decreased GLUT1 levels were found in the AD brain, and overexpression of GLUT1 was shown to ameliorate neuronal activity and metabolism, alleviate cognitive dysfunction, and increase lifespans in an AD mouse model and a Drosophila model $(294,427)$. Crossing transgenic AD mice with GLUT1-deficient mice worsens BBB function and cognitive function (427). Two isoforms of GLUT1 exist: a $55 \mathrm{kD}$ isoform in brain endothelial cells that make up the BBB and a $45 \mathrm{kD}$ isoform in astrocytic endfeet. The capacity of GLUT1 in microvascular membranes of the $\mathrm{BBB}$ is dramatically higher than that in astrocytes (364). Astrocytes are the key cellular support for BBB integrity, and GLUT1 deficiency in endothelia, but not in astrocytes, promotes BBB breakdown (427). GLUT3, mainly expressed in axons and dendrites, is expressed at lower levels in AD patients than in nonaffected individuals and is associated with AD pathology (365). Activation of the cysteine protease Calpain I induces GLUT3 proteolysis and inhibits glucose uptake and O-GlcNAcylation in the AD brain (135).

We and others have shown that fluctuations in glucose disturb metabolic sensors, such as adenosine monophosphateactivated protein kinase (AMPK)-mediated energy metabolism and protein kinase B (Akt, also known as PKB)-mediated insulin signaling in both neurons and peripheral organs, which has been identified to trigger GLUTs to increase glucose metabolism and reduce mTOR to increase autophagy, leading to energy metabolic impairment, cognitive decline, and $\mathrm{AD}$ pathology $(9,60,203,308)$. Glucose metabolic dysfunction leads to advanced glycation end-product (AGE) production. AGEs represent glycated proteins or lipids that play key roles in aging, diabetes, and AD. In patients with AD, AGEs are mostly present in intracellular NFTs. AGEs also affect neurons via the receptor for advanced glycation end products (RAGE), which is an important mediator of $\mathrm{A} \beta$ translocation and Tau hyperphosphorylation via Akt inhibition and subsequent GSK-3 $\beta$ activation (224). Glucose homeostasis can be regulated by the peroxisome proliferatoractivated receptor $\gamma$ (PPAR- $\gamma$ ) coactivator $1 \alpha$ (PGC- $1 \alpha)$, a key molecule for mitochondrial biogenesis, activating gluconeogenic metabolic pathways to attenuate high glucose-induced amyloidogenic processing and promote nonamyloidogenic processing by $\alpha$-secretase through the transcription factor forkhead box O3a (FoxO3a) to influence $\mathrm{A} \beta$ pathology in $\mathrm{AD}$ (318). The intracerebroventricular injection of $\mathrm{A} \beta$ oligomers was shown to trigger peripheral glucose intolerance in transgenic $\mathrm{AD}$ mice, whereas the systemic injection of $\mathrm{A} \beta$ oligomers failed, indicating that $\mathrm{A} \beta$ oligomers in the brain control peripheral glucose homeostasis (61).

The apolipoprotein E4 (ApoE4) genotype is associated with defective glucose utilization in the brains of AD patients (329). A reduced cerebral metabolic rate of glucose utilization is observed in carriers of ApoE4 with AD. Accordingly, high glucose levels were found to be a risk factor for dementia in individuals with the ApoE4 genotype, even among individuals without diabetes (69). In the AD pathological condition, ApoE4 protein fragments escape the normal secretory pathway and enter the mitochondria to bind to the F1-ATPase subunit, thereby reducing energy production. Further, carriers of PS1 gene mutations with FAD have a significantly reduced cerebral glucose metabolic rate, which is associated with a high risk of $\mathrm{AD}$ (281).

This evidence detailed earlier indicates that a decrease in glucose metabolism is an early event in the pathological process of AD. Thus, therapy to improve glucose utilization and metabolism may be a promising approach for the treatment of AD.

\section{B. Insulin resistance}

Insulin is the systematic hormone regulator that participates in metabolic regulation in many tissues. Rising glucose levels throughout the body trigger insulin release to regulate glucose levels in normal homeostasis. The pathological accumulation of glucose in the blood disturbs insulin actions. Insulin in the brain predominantly crosses the BBB from the periphery, and it functions through the tyrosine kinase pathway in neurons and the periphery. Under normal conditions, insulin binds to insulin receptor (IR), followed by the phosphatidylinositol 3-kinase (PI3K)-mediated phosphorylation and activation of Akt, leading to the phosphorylation and inactivation of GSK3 (43).

Insulin in the brain plays a vital role in learning, memory, neurite growth, and development; facilitates glucose metabolism; and affects tau protein and $\mathrm{A} \beta$ processing $(58,67$, 328). An in vivo magnetic resonance imaging study showed that insulin could improve neuronal activity and that increased peripheral insulin levels were associated with brain atrophy and cognition in $\mathrm{AD}$ (38). For example, impaired insulin signaling leads to a decrease in PI3K activity, thereby reducing Akt activity, which is required for neuronal survival, plasticity, and metabolism, and further increasing GSK $3 \alpha / \beta$ activity promotes Tau phosphorylation and $\mathrm{A} \beta$ accumulation (372). In patients with $\mathrm{AD}$, the brain levels of insulin, insulin-like growth factor I (IGF-I), IGF-II, and IGF-I receptor are reduced by nearly $80 \%$ compared with those in age-matched controls (372). In the APP/PS transgenic AD mouse model, the disturbed vessel homeostasis may be due to an imbalance in IGF-I and AMPK angiogenic crosstalk, 
which is reflected by the high IGF-I receptor and p-AMPK levels, impinging cognitive performance in turn (243). Some evidence exists demonstrating that IGF-I modulates the development of $\mathrm{AD}$ in two steps. The first step is the reduction of clearance through IGF-I-modulated carrier proteins, leading to $\mathrm{A} \beta$ accumulation. The second step is that IGF-I dysfunction by amyloidosis disturbs the MAPK and PI3K/ Akt signaling pathways, leading to reduced $\mathrm{A} \beta$ trafficking, reduced glucose uptake, increased cell death, and increased Tau hyperphosphorylation. The evidence was not strong enough to support the therapeutic significance of IGF-I for AD (45). Brain resistance to insulin or IGF-I accounts for neuronal tangle formation and amyloidosis in AD. Insulin and IGF-I are potent neuroprotective factors. AD patients show resistance to IGF-I at the BBB. Insulin/IGF-I, on the one hand, stimulates neuronal $\mathrm{A} \beta$ release. On the other hand, insulin competes with IDE to contribute to extraneuronal $\mathrm{A} \beta$ accumulation (46). Abnormal insulin levels in AD patients repress protein phospholipase $2 \mathrm{~A}$ (PP2A), which is the phosphatase responsible for Tau dephosphorylation. The intraneuronal accumulation of insulin depends on the hyperphosphorylation of Tau, which induces insulin resistance to progress to tauopathy in $\mathrm{AD}$ (338). Intranasal insulin therapy improves memory and cognition and is associated with $\mathrm{A} \beta_{42}$ and $\mathrm{Tau} / \mathrm{A} \beta_{42}$ levels in the cerebrospinal fluid (CSF) of $\mathrm{AD}$, especially in early AD patients, supporting the pharmacologic enrichment of insulin signaling for AD therapy (67).

Insulin resistance is characterized by glucose intolerance and disturbed insulin signaling. Insulin resistance has been confirmed in not only the peripheral tissues of AD patients but also in their brains (385), suggesting that intracerebral insulin signaling impairment may lead to cognitive impairment and that intracerebral insulin resistance may also serve as a biomarker for the risk of neurodegenerative diseases. Insulin resistance appears to be the shared feature of type 2 diabetes and $\mathrm{AD}$, and $\mathrm{AD}$ is also known as type 3 diabetes (2). Peripheral insulin resistance is associated with decreased glucose metabolism in the brain, which, in turn, impairs cognitive function (426). The IDE degrades not only insulin but also $\mathrm{A} \beta$. Intracerebral insulin resistance affects the balance of $\mathrm{A} \beta$ production and degradation by decreasing IDE (104). Genetic studies in Goto-Kakizaki (GK) rats have shown that IDE mutations impair the degradation of $\mathrm{A} \beta$ and induce diabetes (105). IDE expression is much lower in the hippocampi of $\mathrm{AD}$ patients carrying APOE4 than in noncarriers (95). ApoE4 is implicated in the pathways of insulin signaling by their shared mechanism. However, how ApoE4 elicits insulin resistance has not been determined. Possible mechanisms by which ApoE4 elicits insulin resistance include mediating cerebral blood volume and postprandial responses (185), trapping IR in the endosomes (468), and altering DNA hydroxymethylation and metabolic pathways of purine metabolism, glutamate metabolism, and the pentose phosphate pathway (184). Taken together, these studies suggest that IDE and ApoE4 may be the possible link between type 2 diabetes and $\mathrm{AD}$.

As the insulin signaling pathway plays an important regulatory role in both diabetes and $\mathrm{AD}$, insulin resistance may be the key bridge to maintaining crosstalk between peripheral tissues and the brain and may be the first step in the association between AD and diabetes (112). Notably, the antidiabetic drugs rosiglitazone and pioglitazone have been shown to reverse cognitive decline in patients with early $\mathrm{AD}$ by exerting their protective effects on insulin signaling (333, 424). Another antidiabetic drug, exendin-4, which is a stimulator of glucagon-like peptide 1 (GLP-1) receptors, reverses the insulin resistance induced by $\mathrm{A} \beta$ oligomers in the hippocampi of $\mathrm{AD}$ patients and improves behavioral cognition (33). Therefore, understanding the connection and underlying mechanism linking diabetes and $\mathrm{AD}$ may implicate novel and effective therapeutic targets for AD.

\section{Glycosylation}

Impaired glucose metabolism between neurons leads to a disturbance in glycosylation. Glucose metabolism is also involved in the post-translational modification of proteins in the hexosamine synthesis pathway to produce O-Nacetylglucosamine (O-GlcNAc). Two highly conserved enzymes mediate glycosylation, O-GlcNAc transferase (OGT) and O-GlcNAcase (OGA). OGT mediates the addition of O-GlcNAc to serine and/or threonine residues, whereas OGA antagonizes this process and mediates the cleavage of $\mathrm{O}$ GlcNAc. Our studies suggest that enhanced O-GlcNAcylation protects against oxidative stress and promotes mitochondrial homeostasis in aging mice, which provides a possible link between mitochondrial metabolism and $\operatorname{AD}(466,467)$.

Both APP and Tau could be modified by O-GlcNAc modification. Genetic loss of OGT in the mouse forebrain leads to progressive neurodegeneration (408). In AD, deficiencies in GLUT1, GLUT3, and other possible molecules impair glucose metabolism, decrease hexosamine biosynthesis flux, and, subsequently, reduce the production of UDPGlcNAc. The declined UDP-GlcNAc production decreases the O-GlcNAcylation of Tau. The O-GlcNAcylation of Tau inhibits the binding between Tau and microtubules to promote Tau misfolding, which is most likely mediated by CDK5 and GSK-3 $\beta$ (228). The inhibition of OGA increases the O-GlcNAcylation of Tau and decreases NFT formation in the brains and CSF of AD mice, consequently reducing neuron loss $(153,454)$. O-GlcNAcylation and phosphorylation antagonize each other to modify Tau, and reduced levels of O-GlcNAcylation on Tau lead to further hyperphosphorylation, promoting NFTs formation $(229,230)$. Moreover, reduced post-translational modification of O-GlcNAc regulates mitochondrial function, motility, and distribution, suggesting a link between mitochondrial dysfunction and $\mathrm{AD}$ (314). Another type of Tau glycosylation, N-glycosylation, in the AD brain stabilizes the modified Tau structure by facilitating the hyperphosphorylation of Tau by kinases and promoting the suppression of Tau dephosphorylation via phosphatases (413).

Protein kinase A (PKA), a cyclic AMP-dependent kinase, plays a pivotal role in cell metabolism, learning, and memory by activating CREB through phosphorylation in AD. In addition to phosphorylation, the PKA catalytic subunits could also be post-translationally modified by O-GlcNAc. The downregulation of O-GlcNAc thereby regulates PKA-CREB signaling, which, in turn, leads to learning and memory deficits in $\mathrm{AD}$ (441).

The studies described earlier, including those reporting glucose uptake deficiency, insulin resistance, and glycosylation, indicate that impaired glucose metabolism might be the cause rather than the consequence of $\mathrm{AD}$ (Fig. 3). 


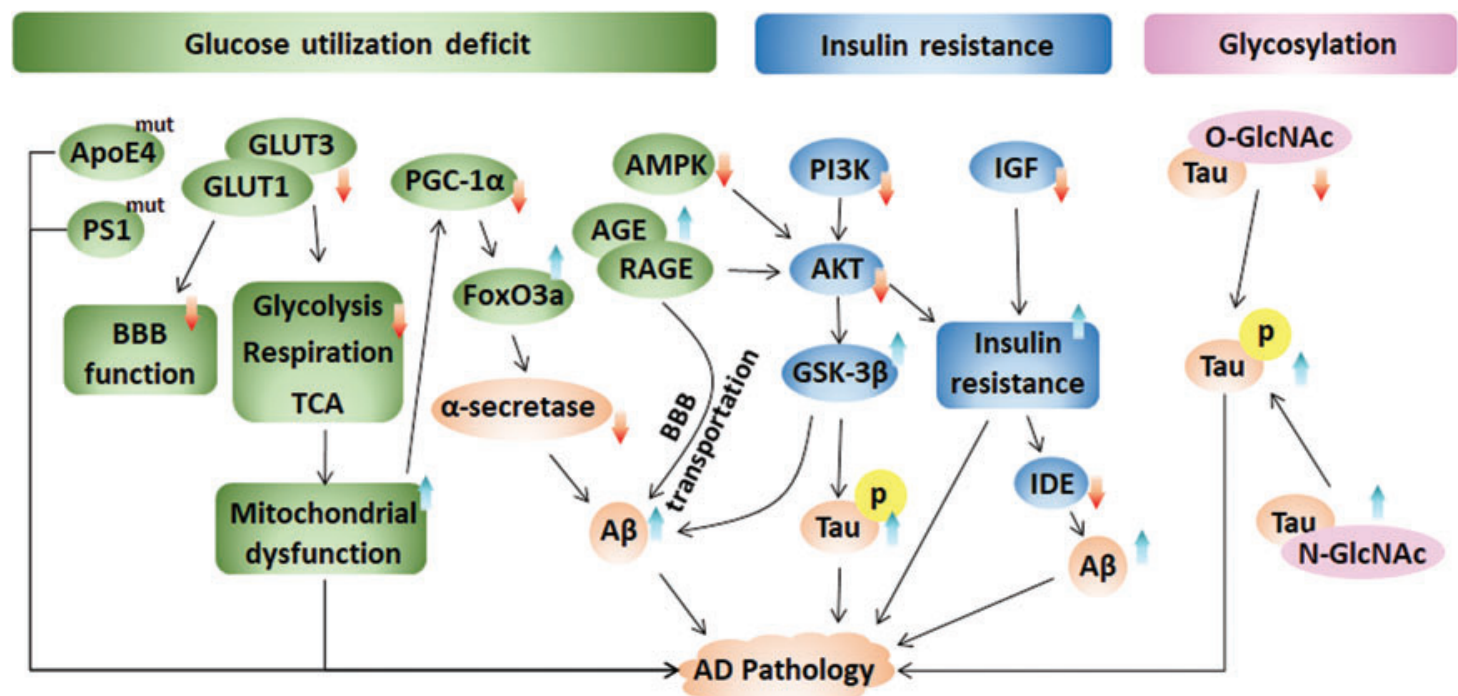

FIG. 3. Periphery and central glucose metabolic disorders contribute to AD pathology. Glucose metabolism is impaired in the early stage before plaque deposition and progresses along with cognitive decline, further affecting AD pathology. (i) Glucose uptake deficit. The ApoE4 genotype and PS1 gene mutant are associated with defective glucose utilization in the brains of AD patients. GLUT1 and GLUT3 are major glucose transporters that control the function of the $\mathrm{BBB}$ and mitochondrial functions, including glycolysis, respiration, and the TCA cycle. Fluctuation in glucose disturbs the metabolic sensor AMPK, which further affects AKT-mediated insulin signaling. Further, PGC-1 $\alpha$, which can be inhibited by mitochondrial dysfunction, diminishes $\alpha$-secretase through FoxO3a to influence A $\beta$ pathology in AD. AGE interacts with its receptor RAGE to mediate $\mathrm{A} \beta$ translocation and enhance Tau hyperphosphorylation via AKT inhibition and subsequent GSK-3 $\beta$ activation. (ii) Insulin resistance. Rising glucose levels throughout the body trigger insulin release to regulate glucose levels and achieve normal homeostasis. Impaired insulin signaling decreases PI3K activity, thereby reducing AKT activity (which can also be decreased by AMPK), and further increases the activity of GSK-3 $\alpha / \beta$, which then phosphorylates Tau and promotes $\mathrm{A} \beta$ deposition. IGF induces insulin resistance, which affects the balance of $\mathrm{A} \beta$ production and degradation by decreasing IDE. (iii) Glycosylation. O-GlcNAcylation and phosphorylation inversely modify the Tau protein, whereas N-glycosylation stabilizes the modified Tau structure. AGEs, advanced glycation end-products; AKT, protein kinase B (also known as PKB); AMPK, adenosine monophosphate-activated protein kinase; ApoE4, apolipoprotein E4; $\mathrm{BBB}$, blood-brain barrier; FoxO3a, forkhead box O3a; IDE, insulin degrading enzyme; IGF, insulin-like growth factor; PGC- $1 \alpha$, peroxisome proliferator-activated receptor $\gamma$ coactivator $1 \alpha$; PI3K, phosphatidylinositol 3-kinase; RAGE, receptor for advanced glycation end products. Color images are available online.

\section{Lipid Metabolism in AD}

The adult brain is highly enriched in lipids, which account for $\sim 20 \%-25 \%$ of the dry weight. These lipids not only constitute the structure of membranes but also provide the infrastructure and function for the membrane system (428). Basically, lipids participate in cell membrane formation, cellular transportation, energy storage, and signal transduction (428). In the liver, insulin signaling controls lipid metabolic events, including stimulating lipogenesis and reducing lipolysis.

Alois Alzheimer first recorded remarkable lipid granule accumulation in the glia of his patient Auguste Deter (108). Altered lipid composition in the cell membranes leads to redistribution of proteins linked to the $\beta$-amyloidogenic pathway of lipid rafts in AD (80). The cleavage of APP by the $\beta$-secretase protein BACE1 and $\gamma$-secretase proteins, including PSEN1, PSEN2, aph-1, pen-2, and nicastrin, occurs in lipid rafts and affects lipid metabolic enzymes, thereby altering subcellular trafficking. Frontal cortex lipid rafts are greatly altered in $\mathrm{AD}$ brains from the initial neuropathologic stages of $\mathrm{AD}$, but the underlying mechanism is unknown. However, the changes in lipid rafts were shown to affect both lipid classes and fatty acids, increase the membrane order and viscosity, providing a favorable lipid raft microenvironment for the APP/BACE1 interaction and the amyloidogenic pathway and strengthening AD as a result (100). An age-dependent modification was found in the lipid raft microdomains, thereby affecting signal transduction and protein-protein interactions. The alterations were more dramatic and occurred earlier in APP/PS1 mice (101). Lipid metabolism imbalance also plays a critical role in $\mathrm{AD}$ pathogenesis. Oxidative damage to lipids by ROS under pathological AD conditions also leads to lipid peroxidation, and lipid peroxidation products and $\mathrm{A} \beta$ plaques are colocalized in the brain. Taken together, these data suggest that lipid dysregulation is a major risk factor for $\operatorname{AD}(99,201,226,412)$ (Fig. 4).

\section{A. Lipid dysregulation as an early signal in $A D$}

Lipid peroxidation occurs as an early event in the progression of AD (265), suggesting that the early prevention of 


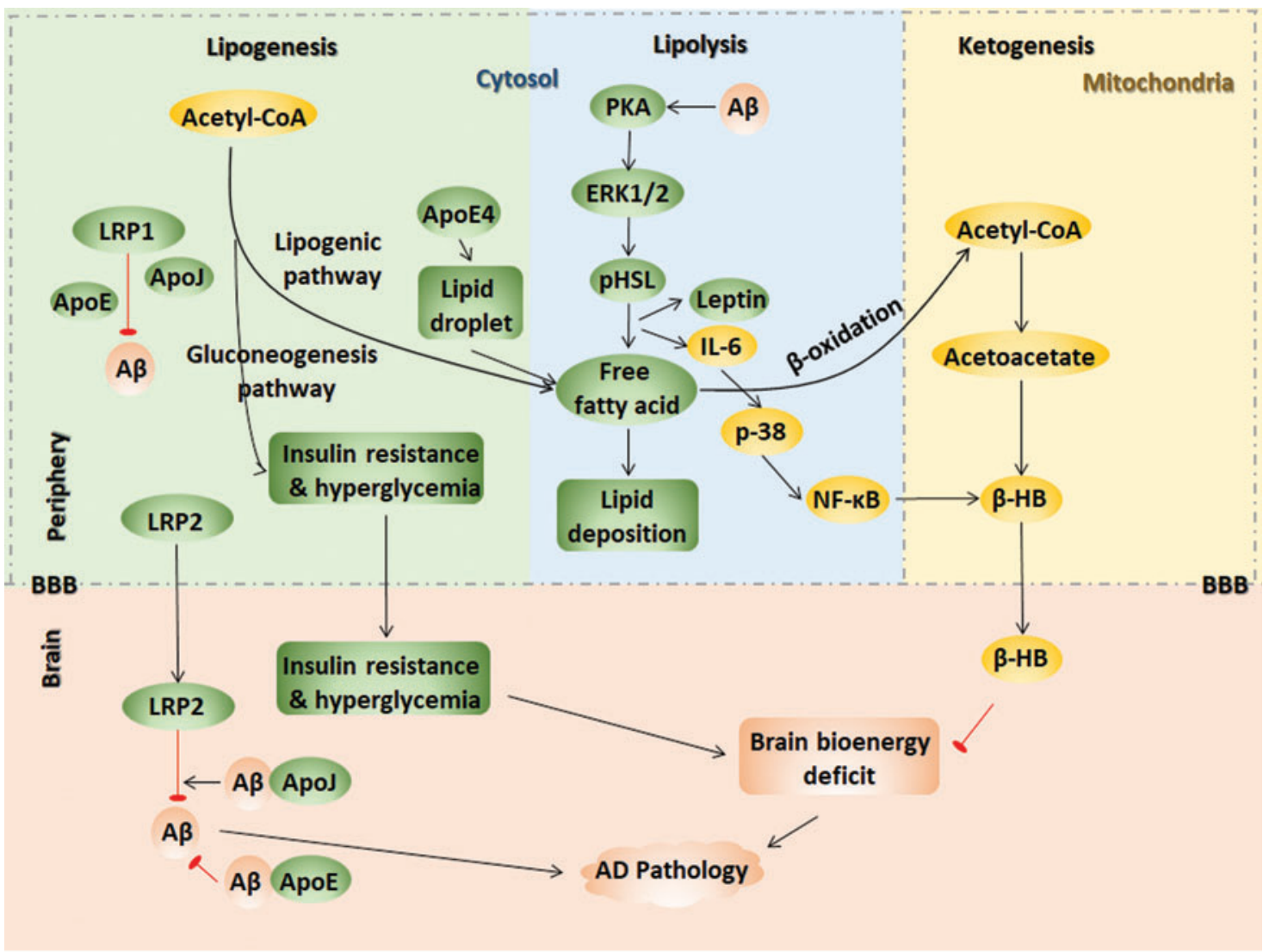

FIG. 4. Peripheral and central lipid dysmetabolism contributes to AD pathology. Lipid dysregulation is a major risk factor for AD. (i) Lipogenesis. LRP2 clears A $\beta$ across the BBB by ApoE- and ApoJ-mediated $\mathrm{A} \beta$ crosstalk. Peripheral A $\beta$ clearance might be mediated by ApoE, ApoJ, and LRP1. Early inflammation in AD inactivates the lipogenic pathway to initiate the substrate flux for glucose production, leading to severe hyperglycemia and insulin resistance. ApoE4 increases the number of lipid droplet and enhances fatty acids oxidation. (ii) Lipolysis. A $\beta$ stimulates lipolysis via the PKA and ERK1/2 pathways, phosphorylating HSL and stimulating leptin and IL-6 secretion, resulting in fatty acid release to trigger lipid deposition and insulin resistance. (iii) Ketogenesis. Acetyl-CoA is the substrate of acetoacetate, which finally produces $\beta$-HB. IL-6-induced $\mathrm{p} 38 / \mathrm{NF}-\kappa \mathrm{B}$ activation also induces $\beta$-HB production. $\beta$-HB crosses the $\mathrm{BBB}$ to serve as brain bioenergy instead of glucose under hypometabolic conditions in AD. The brain bioenergy deficit induced by insulin resistance and hyperglycemia aggravate AD pathology. ERK1/2, extracellular signal-regulated kinase 1/2; HSL, hormone sensitive lipase; NF- $\kappa \mathrm{B}$, nuclear factor-kB; PKA, protein kinase A. Color images are available online.

lipid peroxidation products in the presymptomatic phase of AD or before mild cognitive impairment (MCI) could be an effective way to prevent AD. Indicators of lipid peroxidation include thiobarbituric acid reactive substances, Notably, 4hydroxynonenal and acrolein are elevated in the AD brain (244). Ten lipid metabolites derived from lipidomic approaches have been validated as biomarkers of preclinical AD in blood that could distinguish cognitively normal individuals from cognitively impaired individuals with an accuracy above $90 \%$ (264). The biomarkers for $\mathrm{AD}$ will be summarized in part V, titled "Mitochondria-centered Metabolic Markers in the Early Diagnosis of AD."

Lipid-based supplementation has the potential to reduce the risk of AD. Docosahexaenoic acid (DHA), which is a prominent polyunsaturated $\omega-3$ fatty acid efficiently transported across the BBB for the formation of a neuronal membrane structure, could increase the $\alpha$-secretase cleavage of nonamyloidogenic processes and decrease the amyloidogenic processing activities of $\beta$-secretase and $\gamma$-secretase. An increased DHA level was found in the early stage of AD by the neurolipidomic study in human AD cases and the DHA levels are progressively manifested as the trilogy of adaptation-overload-failure, leading cells to neurodegeneration (289). DHA serves as an endogenous ligand for retinoic acid receptor and retinoid $\mathrm{x}$ receptor, which are responsible for memory function and neurogenesis. DHA activates $\mathrm{Ca}^{2+}$ / calmodulin-dependent protein kinase II (CaMKII), which maintains long-term potentiation in the hippocampus, thereby mediating learning and memory functions. DHA increases brain-derived neurotrophic factor (BDNF) in the hippocampus by activating PI3K/Akt signaling to protect synaptic plasticity and nerve cell survival (5). Inflammatory markers contributing to $\mathrm{AD}$ can also be reduced by DHA supplementation. A deficiency of DHA in neurons leads to neurodegeneration and cognitive dysfunction. Dietary supplementation with DHA in early stage reverses $\mathrm{A} \beta$ oligomerization in the animal model (389); however, this strategy has failed in several clinical trials $(109,322)$.

DHA intervention has beneficial effects only in the early stage of $\mathrm{AD}$. DHA was shown to enhance the binding 
between $\mathrm{A} \beta_{42}$ and lipid rafts to promote $\mathrm{A} \beta$ degradation. IDE secretion and activity can be increased by DHA, thereby ameliorating $\mathrm{AD}$ pathology by increasing $\mathrm{A} \beta$ turnover (132).

ApoE4 is a major risk factor for AD and is associated with a high cholesterol level, as ApoE4 delivers cholesterol from astrocytes to neurons, indicating disrupted lipid metabolism in $\mathrm{AD}$ (330). ApoE and cholesterol colocalize with $\mathrm{A} \beta$ in the brains of transgenic AD mice and AD patients, and cholesterol is reportedly an early risk factor for $\mathrm{AD}(215,302)$. ApoE binds to $\mathrm{A} \beta$ in a lipid-dependent manner, and $\mathrm{A} \beta$ deposition increases in ApoE mice (212). The lipid-binding region on ApoE4 mediates the neuro-mitochondrial toxicity of ApoE4 (50). The ApoE4 genotype is established in carriers $\sim 20-39$ years of age, and these carriers have functional brain abnormalities as relatively young adults, several decades before the onset of dementia (330). Before the onset of AD, ApoE4 carriers supplemented with high-dose DHA showed a reduced risk of developing $\mathrm{AD}$ symptoms (450). Recent evidence suggests that single nucleotide polymorphisms in the genes St-AT-related lipid transfer domain 6 (STARD6) and near enoyl CoA-hydratase domain containing 3 (ECHDC3) are responsible for the lipid level and metabolism in AD, and variants in or near the STARD6 and ECHDC3 genes, especially ApoE4 carriers, are risk factors associated with AD (452). The role of ApoE4 in lipid metabolism and AD indicates a lipid-driven mechanism of $\mathrm{AD}$ pathogenesis.

\section{B. Lipogenesis in $A D$}

Lipogenesis is the biochemical process that converts acetyl CoA into fatty acids, which primarily occurs in the liver. The increased lipoprotein secretion in the periphery aggravates the $\mathrm{A} \beta$ burden in the $\mathrm{AD}$ brain. Lipoprotein receptor-related protein 2 (LRP2) clears $\mathrm{A} \beta_{40}$ rapidly across the $\mathrm{BBB}$. Other lipid proteins, such as $\mathrm{ApoE}$ and $\mathrm{ApoJ}$, mediate $\mathrm{A} \beta$ crosstalk between the brain and periphery. High ApoE-A $\beta$ binding activity decreases $\mathrm{A} \beta$ brain efflux, whereas ApoJ-A $\beta$ binding increases LRP2-mediated efflux. The poorly lipidated ApoE increases the $\mathrm{A} \beta$ deposition. Alternatively, the lipidation status of peripheral $\mathrm{A} \beta$ may also affect its clearance, which, in turn, might also be mediated by ApoE, ApoJ, and LRP1 (66). The ApoE4-driven disruption in fatty acid metabolism is proposed to be factor underlying why ApoE4 carriers are at a high risk for developing AD. ApoE4 in astrocyte exhibits an increase in the number of lipid droplet, which serves to sequester fatty acids depending on the saturation status of the fatty acid. The expression of the lipid droplet marker PLIN2 and the oxygen consumption rate from endogenous fatty acid oxidation are also enhanced in E4 astrocytes (102). Proteins involved in mitochondrial fatty acid oxidation, including PTEN-induced putative kinase 1 (PINK1) and PGC- $1 \alpha$, were shown to be cooperatively downregulated in $\mathrm{AD}$ and diabetic patient brains, indicating a role of mitochondria in linking $\mathrm{AD}$ and diabetes (57).

A high-fat diet (HFD) increases the risk of AD, a phenomenon that is related to dysfunctional lipid metabolism. Abnormal lipid accumulation leads to insulin resistance and vice versa. Depleting the negative insulin signaling regulator PTEN results in a fatty liver and induces lipogenesis (421). Our previous study compared C57 mice with APP/PS1 transgenic AD mice, showing that the HFD induced more severe body weight gain, hyperglycemia, and hepatic insulin resistance in $\mathrm{AD}$ mice than in $\mathrm{C} 57$ mice, potentially because an inactivated lipogenic pathway by inflammatory factors initiates the substrate flux for glucose production, leading to more severe hyperglycemia in AD mice $(273,387)$. Hepatic insulin resistance in diabetic $\mathrm{db} / \mathrm{db}$ mice was intensified by hybridization with AD mice (384). Early inflammation induced by an HFD in APP/PS1 mice resulted in less hepatic steatosis than that in C57 mice that were also fed an HFD (387).

\section{Lipolysis in $A D$}

Under energy-demanding conditions, adipose tissue begins to undergo lipolysis, which activates the PKA and extracellular signal-regulated kinase 1/2 (ERK1/2) signaling pathways that phosphorylate hormone-sensitive lipase, resulting in free fatty acid release (96). The activity of lipolytic enzymes, including monoacylglycerol and diacylglycerol, is stimulated in plasma membrane and synaptosomal plasma membrane fractions obtained from various regions of normal and AD brains (103).

APP can be processed to produce the $\mathrm{A} \beta$ peptide in adipose tissue, wherein the $\mathrm{A} \beta$ peptide stimulates lipolysis and adipokine secretion via PKA and ERK1/2-dependent pathways and stimulates the secretion of leptin and IL-6, resulting in the release of free fatty acids and proinflammatory adipokines (407). Elevated fatty acid levels trigger lipid deposition and insulin resistance, demonstrating a close relationship between energy metabolism and AD.

\section{Ketogenesis in $A D$}

In the ketogenesis pathway, acetoacetyl-CoA is generated by acetyl-CoA, which is mediated by thiolase, and $\beta$-hydroxy- $\beta$-methylglutaryl-CoA (HMG-CoA) is then generated by another acetyl-CoA that is mediated by HMG-CoAsynthase. HMG-CoA generates acetyl-CoA, and acetoacetate is mediated by HMG-CoA lyase. The final product D- $\beta$ hydroxybutyrate $(\beta-\mathrm{HB})$ derives from the reaction between acetoacetate and $\mathrm{NADH}$, which is mediated by $\beta$-hydroxybutyrate dehydrogenase 1 (BHD1) (317). Therefore, acetoacetate and $\beta$-HB are two main ketone bodies. Ketone bodies are produced by the liver from fatty acids. Fatty acids, responsible for providing cellular energy to peripheral tissues, are oxidized in the liver, producing large amounts of acetyl-CoA, surpassing the ability of TCA to synthesize ketone bodies in liver mitochondria. Then, ketone bodies are transported to extrahepatic tissue through the blood for oxidation and the subsequent release of acetylCoA, which enters the TCA cycle, thereby generating energy $(119,205)$. Ketone bodies decrease the need for glycolysis and the mitochondrial $\mathrm{NAD}^{+} / \mathrm{NADH}$ ratio. Using label-free quantitative proteomics, SIRT3 was found to target each complex in the TCA cycle, as does every enzymatic step from fatty acids to ketone body $\beta$-HB. The mitochondrial deacetylase SIRT3 regulates acetylation via ketogenesis during trafficking to the mitochondrial matrix, maintaining the balance of energy homeostasis (323).

Although the brain mainly uses glucose as the sole energy substrate under normal conditions, under hypometabolic conditions in $\mathrm{AD}$, ketone bodies, such as acetoacetate or $\beta$-HB, may be used as an alternative energy substrate for glucose. $\beta$-HB is the most abundant ketone, constituting $\sim 70 \%$ of ketone bodies primarily formed in liver mitochondria and 
then transported by blood to other tissues. $\beta$-HB-enriched ketogenic diets could be helpful for $\mathrm{AD}$ intervention, which will be discussed in part VI, titled "Mitochondrial Metabolic Dysfunction as Target for AD Prevention."

In the $\mathrm{AD}$ mouse model (APP/PS1/Tau, $3 \times \mathrm{Tg}$ ), the enzymes responsible for ketone body metabolism were significantly increased (447). In early $\mathrm{AD}, \mathrm{A} \beta$ invades the mitochondria, initiating their production of superoxide radicals and the conversion of these molecules into hydrogen peroxide. These events lead to oxidative stress and activation of the C-Jun NH2-terminal kinase (JNK)-MAPK pathway and nuclear factor- $\mathrm{kB}(\mathrm{NF}-\kappa \mathrm{B})$, resulting in the release of proinflammatory interleukins and cytokines $(8,321)$. We recently reported that the hepatic proinflammatory cytokine IL-6 activates 3-hydroxy-3-methylglutaryl-CoA synthase 2, which then functions as a critical regulator via $\mathrm{p} 38 / \mathrm{NF}-\kappa \mathrm{B}$ p65 signaling in inflammation-induced ketogenesis in the early stage of AD (359). This finding implies that as a selfadaptation mechanism, cerebral glucose metabolic dysfunction in early AD increases the ketone body supply from the liver to the brain, resulting in a shift to ketone body utilization (359). During the early stage of $\mathrm{AD}$, the constant activation of ketogenesis could maintain mitochondrial function to prevent and/or delay further development of the disease. Enriched ketogenesis in the liver and ketogenic diets may help prevent or delay a bioenergetic decline in the early AD brain (449). It remains unclear which event in AD triggers a hepatic inflammatory response, such as IL-6 accumulation.

\section{Mitochondria at the Metabolic Core in AD}

\section{A. Mitochondrial dysfunction precedes metabolic disorder in $A D$}

Mitochondria are crucial organelles for energy metabolism and signal cascade control. Metabolism of glucose, lipogenesis, and ketogenesis occurs in mitochondria, and mitochondrial dysfunction tends to be the cause or effect of metabolic dysfunction. The abnormalities in $\mathrm{AD}$, including defective glucose metabolism and energy metabolism, suggest that mitochondrial dysfunction is involved in $\mathrm{AD}$ pathology and is an early prominent feature. Studies by ourselves and others have demonstrated reduced activities of enzymes mediating mitochondrial respiration and the TCA cycle in the $\mathrm{AD}$ brain and periphery, including succinate dehydrogenase (SDH) or complex II, COX or complex IV, pyruvate dehydrogenase complex (PDC), and $\alpha$-ketoglutarate dehydrogenase complex (KGDH), confirming the link between energy metabolism dysfunction and mitochondrial dysfunction in $\mathrm{AD}(242,377)$. Genetic mutations have been identified in mitochondrial-encoded cytochrome c oxidase subunits I, II, and III (COX-1, 2, and 3) in AD.

Mitochondria are very sensitive to extracellular glucose changes in both the brain and periphery, and changes in mitochondrial function precede AMPK/Akt signal fluctuations. In addition, studies by ourselves and others have also demonstrated that AMPK inactivation leads to PI3K-mediated insulin resistance and PGC- $1 \alpha$-mediated mitochondrial loss in both peripheral organs and the brain, resulting in disorders of glucose metabolism and neuronal damage that are ameliorated by normal glucose metabolism and mitochondrial activity, providing new insights into the interrelationship between hyperglycemia and cognitive impairment (140, 308). Embryonic neurons derived from the AD mouse hippocampus exhibited a significant decrease in mitochondrial respiration and an increase in glycolysis (448).

In addition, mitochondrial dysfunction is greater in ApoE4 carriers than in AD patients who do not carry ApoE4 (123). ApoE4 is also associated with decreased cerebral glucose metabolism in $\mathrm{AD}$, indicating that a combination of cerebral metabolic rates and genetic risk factors offers a strategy for preclinical AD diagnoses (368). The level of $\alpha$-ketoglutarate dehydrogenase, a mitochondrial enzyme in the TCA cycle, is decreased in the AD patient brain, particularly in those of ApoE4 carriers (123). The truncated ApoE4 elicits mitochondrial function and integrity, indicating that blockage of the interaction between mitochondria and ApoE4 is a potential treatment strategy (50).

As mitochondria are the site of the TCA cycle and respiration, mitochondrial dysfunction is a major intracellular event that contributes to AD pathology. This event occurs early in $\mathrm{AD}$ progression, even before the onset of $\mathrm{A} \beta$ pathology, making it critical to develop strategies aimed at mitochondrial function $(257,259,327)$.

\section{B. Mitochondria mediate pathological impairment during $A D$ progression}

Decreases in respiratory capacity, increases in mitochondrial fragmentation, and fractures in the mitochondrial cristae structure occurs in the AD brain, and the abnormalities in mitochondria appear before pathological $\mathrm{A} \beta$ plaque deposition $(371,438)$. A $\beta$ accumulates exclusively in mitochondria. The mitochondrial membrane 40 import channel and mitochondrial membrane 23 channel were shown to bind to $\mathrm{A} \beta$ and cause mitochondrial dysfunction in the human AD brain but not in the age-matched controls (78). A $\beta$ binds exclusively to $\mathrm{ADH}$. The $\mathrm{A} \beta$ and $\mathrm{ABAD}$ complex promotes freeradical generation and mitochondrial dysfunction, interacts with mitochondrial components, impairs ATP production, and induces oxidative stress in $\mathrm{AD}$, which could be the underlying mechanism of $A \beta$ toxicity in mitochondria (248).

Cyclophilin D (CypD), an integral part of the mPTP, directly interacts with $\mathrm{A} \beta$ in mitochondria, promotes ROS generation, and mediates mPTP formation, resulting in mitochondrial stress-induced neuronal cell death in $\mathrm{AD}$ mice and patients (258). Moreover, the deficiency of CypD improves learning and memory in $\operatorname{AD}$ mice $(84,258)$. As the direct site of $\mathrm{A} \beta$ accumulation in $\mathrm{AD}$ neurons, mitochondria are responsible for ROS generation and oxidative damage. The A $\beta$ peptide produced by APP splicing binds to ferroheme, resulting in the decreased activity of complex IV. Using the complex IV inhibitor $\mathrm{NaN}_{3}$, the accumulation of APP and the production of amyloid C-terminal fragments are greatly enhanced. In addition, the $\mathrm{A} \beta$ peptide stimulates GSK $3 \beta$ activity, phosphorylates pyruvate dehydrogenase, and finally inhibits energy metabolism. Hydrogen peroxide is increased and cytochrome oxidase activity is decreased before the appearance of $\mathrm{A} \beta$ plaques, suggesting that early mitochondria-targeted therapeutic interventions might be an effective strategy in delaying AD progression and treatment (257). Mitochondrial dysfunction also induces synaptic damage, impairs neurotransmission, and causes AD-related cognitive decline $(225,326,348)$. 


\section{Mitochondrial remodeling in $A D$}

Mitochondria are highly dynamic organelles that continuously, morphologically, and functionally undergo two opposing processes, namely, fission and fusion (15). Mitochondrial fission is controlled by the GTPase protein dynamin-related protein 1 (Drp1). Drp1 interacts with its receptors mitochondrial fission 1 (Fis1) and mitochondrial fission factor to stimulate mitochondrial fission (49). On the other hand, mitochondrial fusion is controlled by other GTPase proteins, optic dominant atrophy 1 (OPA1), mitofusin 1 (Mfn1) and mitofusin 2 (Mfn2) (48). It has been shown that excessive fission leads to mitochondrial fragmentation and the imbalance between fission and fusion compromises mitochondrial respiration function and ATP production (433). Besides, impaired mitophagy disturbs the clearance of defective mitochondria, which leads to the production of ROS and causes oxidative stress (198).

The levels of proteins governing mitochondrial fission, including Drp1 and Fis1, and mitochondrial fusion, including OPA1, Mfn1, and Mfn2, are significantly altered in AD mice and patients $(258,419)$. Besides the change in mitochondrial morphology, the distribution of mitochondria in neuron and axis is altered in AD patients (419). The distribution of mitochondria in neuron is a very complex topic. Inhibition of Drp1 in AD mouse neurons prevents cognitive decline, mitochondrial fragmentation, lipid peroxidation, BACE1 expression, and $\mathrm{A} \beta$ deposition (19). In addition to $\mathrm{A} \beta$-related $\mathrm{AD}$ pathology, Drp1 reduction also protects against hyperphosphorylated Tau and phosphorylated Tau-induced mitochondrial dysfunction in AD (189). The S-nitrosylation of Drp1 at Cys644 causes A $\beta$-related mitochondrial fission and neuronal injury, leading to mitochondrial fragmentation in human $\mathrm{AD}$ neurons (56). Treatment of mitochondrial division inhibitor 1 (mdivi-1), a mitochondrial fission inhibitor, markedly improves mitochondrial dynamics and cognitive function in $\mathrm{AD}$ mouse model CRND8 early before the accumulation of amyloid pathology (417).

Similarly, manipulation of mitochondrial fusion also plays a role in AD. For example, OPA1 overexpression alleviates mitochondrial ROS overproduction in the AD brain (419). PS2 mutation in FAD promotes mitochondria and endoplasmic reticulum (ER) coupling in the presence of Mfn2. The PS2-Mfn2 binding is more efficient in mitochondriaassociated membranes (107). Further, the activity of PS2 is affected by Mfn2 knockdown-increased mitochondria-ER coupling, leading to the decrease of $\mathrm{A} \beta$ accumulation (216).

Mitochondrial metabolic homeostasis is maintained by the balance between mitochondrial biogenesis and degradation. PGC- $1 \alpha$ is a transcriptional coactivator that regulates mitochondrial biogenesis through the downstream factors peroxisome proliferator-activated receptors (PPARs), nuclear respiratory factor $1(\mathrm{NRF} 1)$, and nuclear respiratory factor 2 (NRF2), which govern the nuclear genes encoding mitochondrial proteins, and mitochondrial transcription factor A (TFAM), which initiates mtDNA transcription and replication (434). The evolutionarily conserved form of degradation is autophagy, which is responsible for the degradation of accumulated and cytotoxic proteins. Autophagy facilitated in mitochondria is called mitophagy, and energy deficits initiate autophagic pathways. The molecular interaction between autophagy and $\mathrm{AD}$ pathology remains controversial. For example, the triggered autophagy pathway increases the degradation of the Tau protein and excessive $\mathrm{A} \beta$ accumulation in early AD. However, autophagosome accumulation exacerbates AD pathogenesis (140). More in-depth studies are needed to clarify the mechanisms by which molecules participate in autophagy pathway functions through $\mathrm{AD}$ progression, and the function of different molecules may be stage dependent in AD progression.

FOXO transcription factors are essential for memory and neurodegeneration, for the manipulation of glucose and lipid metabolism, and for autophagy in response to energy deprivation, providing a potential link between $\mathrm{AD}$ and energy deficits. FoxO3a, which induces LC3b, Beclin1, and Bnip3 for autophagy and PINK1 for mitophagy, is an important transcription factor for mitochondrial gene expression and ROS production. We found that AMPK, as an energy sensor, directly phosphorylates FoxO3 under energy deficient conditions (238). The decreases in FoxOs expression in the brain insulin resistance state are related to toxic $\mathrm{A} \beta$ formation and Tau hyperphosphorylation in diabetic transgenic mice and monkeys (340). However, other studies have demonstrated that the consecutive activation of FoxO proteins induces neuronal loss, $\mathrm{A} \beta$ deposition, and Tau hyperphosphorylation (263). In addition, in patients with AD, impaired glucose metabolism decreases PGC- $1 \alpha$ levels and then increases FoxO3a levels, inhibiting $\alpha$-secretase in the nonamyloidogenic processing of APP, thereby promoting amyloidogenic processing to produce excessive $\mathrm{A} \beta(318,354)$.

Although more investigations are needed to determine the mechanism by which the dynamic state of mitochondria eliminates or aggravates $\mathrm{AD}$, the current findings suggest that the steady state of mitochondrial remodeling in AD supports its early and fundamental role in AD-related pathological and cognitive impairments, suggesting abnormal mitochondrial remodeling as a possible therapeutic target for halting $\mathrm{AD}$ (Fig. 5). Nutrients targeting mitochondria-related signals may be a new strategy for AD prevention and treatment and will be discussed in part VI-A.

\section{Mitochondria-induced inflammation and oxidative stress during the pathogenesis of $A D$}

The brain is especially vulnerable to oxidative stress because of its high oxygen needs. Oxidative stress arises from excess ROS, most of which are generated from mitochondria by electron leakage from the OXPHOS and TCA cycle and are also generated from pathological AD conditions (110, 404, 420). Mitochondrial dysfunction in neurons leads to insufficient energy supply and the release of a large amount of ROS, which induces oxidative stress and the imbalance of calcium regulation, ultimately triggering neuronal apoptosis. Brain oxidative stress linked to mitochondrial dysfunction arises in early $\mathrm{AD}$ and contributes to $\mathrm{AD}$ pathogenesis, and the extent of oxidative damage is much higher in mtDNA than in nuclear DNA (276). In 3-month-old transgenic APP mice, mitochondria in the brain have shown decreased membrane potential, COX activity, and ATP levels. At this time, the $\mathrm{A} \beta$ levels in neurons are increased, whereas extracellular $\mathrm{A} \beta$ deposition does not yet occur, suggesting that mitochondrial dysfunction occurs earlier than $\mathrm{A} \beta$ pathological deposition. ROS-mediated Akt1 oxidative modification causes synaptic dysfunction, which occurs very early in the AD process (4). 


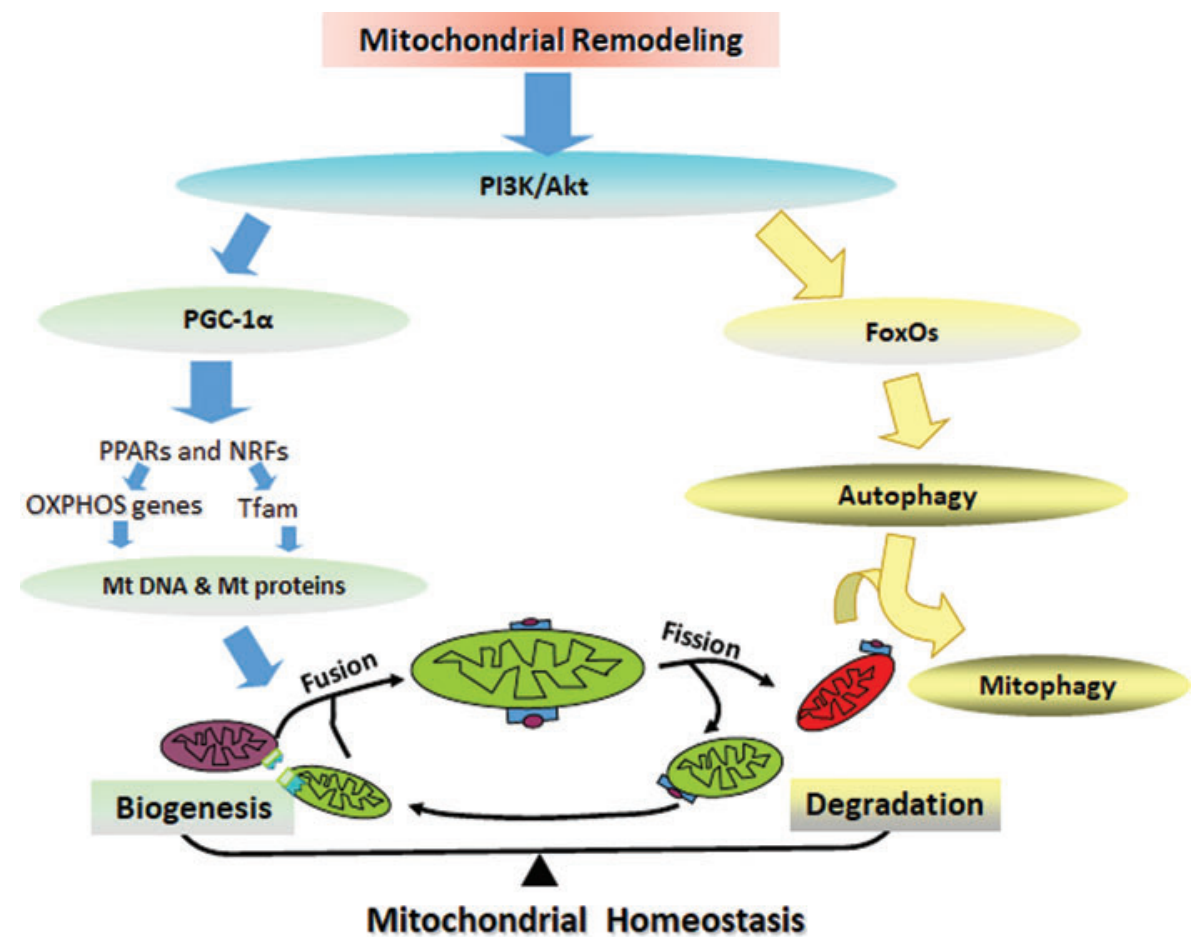

FIG. 5. Mitochondrial remodeling in AD. Metabolic dysfunction in AD precedes PI3K/AKT signal fluctuation, which then functions through PGC- $1 \alpha$ or FoxO signaling. PGC- $1 \alpha$ regulates mitochondrial biogenesis though the downstream factors PPARs and NRF1s, which govern the OXPHOS genes encoding mitochondrial proteins and TFAM, thus initiating mtDNA transcription and replication. FoxOs manipulate autophagy and mitophagy in response to energy deprivation. Mitochondria are highly dynamic organs undergoing fission and fusion continuously, morphologically, and functionally to balance mitochondrial fragmentation. The balance between mitochondrial biogenesis and degradation maintains mitochondrial metabolic remodeling. More investigations are needed to determine the underlying mechanism of how mitochondrial dynamic regulates AD. mtDNA, mitochondrial DNA; NRF1, nuclear respiratory factor 1; PPARs, peroxisome proliferator-activated receptors; TFAM, mitochondrial transcription factor A. Color images are available online.

Mitochondria are the hub of the redox system. The overall mitochondrial redox status can be elucidated by fluorescence imaging or HPLC. Free radical oxidation of polyunsaturated fatty acids, cholesterol, and glucose causes overload of toxic products, leading to the destruction of cellular redox homeostasis (55). Nrf2, a redox-sensitive transcriptional factor, is regulated by Keap1 and controls the expression of antioxidant genes through interactions with antioxidant response element (ARE) to balance the endogenous antioxidant stress defense system. Basically, Nrf2 binds to Keap1 with the E3 ubiquitin ligase Cullin 3-RING box1 for ubiquitination, resulting in proteasomal degradation. Without Keap1's target, Nrf2 binds to ARE to activate the transcription of the phase 2 response. Nrf2 and ARE levels decrease on increased $\mathrm{A} \beta$ plaque deposition in APP/PS1 transgenic mice. The $\mathrm{A} \beta$ toxicity could be decreased by enhancing Nrf2-ARE activity.

In addition, the activation of Nrf 2 reduces Tau phosphorylation, suggesting that the activation of an Nrf2-ARE antioxidant defense has a neuroprotective effect and may prevent or delay the pathological changes in AD. A study in another $\mathrm{AD}$ mouse model confirmed that oxidative damage precedes $\mathrm{A} \beta$ and further demonstrated that oxidative damage increases the deposition of $\mathrm{A} \beta$ in the mouse brain. A mitochondrial superoxide dismutase (MnSOD) deletion in APP mice significantly increased $\mathrm{A} \beta$ levels and amyloid deposits in the brain $(39,247,282)$. These findings suggest that oxidative stress exists before the characteristic features of AD pathol- ogy, such as $\mathrm{A} \beta$ deposition, and promotes $\mathrm{A} \beta$ deposition during AD progression.

Oxidative stress triggers the JNK-MAPK signaling pathway, leading to an inflammatory response and inflammasome activation (307). A $\beta$ increases the production of the inflammatory cytokine TNF- $\alpha$, causing insulin resistance by JNK in the AD mouse hippocampus (33). Activated JNK was found in the AD brain and periphery (33). Oxidative stress promotes the transcriptional activity of FoxO, which induces JNK activation. As JNK signaling pathways affect mitochondrial function and mitochondrial bioenergetics, it is reasonable to link mitochondria, ROS, and inflammation together with AD. In AD, excessive ROS contributes to insulin resistance and inflammasome activation mostly through mitochondria $(360,474)$. Oxidative stress and inflammation in early AD damage the neuronal membrane potential, mtDNA, TCA cycle, and electron transport chain in mitochondria, thereby reducing ATP generation and exacerbating oxidative damage (343, 448).

In $\mathrm{AD}$, increased peripheral inflammation occurs early in the MCI phase and decreases as a function of the severity of AD. The release of inflammatory cytokines and chemokines improves the generation of fatty acids, lipid peroxidation products, and ROS. Our studies identified higher hepatic and serum TNF- $\alpha$ and IL- 6 contents in the early stage of AD in transgenic mice before the activation of these inflammatory molecules in the brain $(359,387)$. Inflammatory-related genes are 
increasingly featured in AD genetic studies, and inflammatory cytokines even play a role as peripheral biomarkers to some extent. Thus, there appears to be an early response to abnormal protein deposition that develops into immune adaptation in chronic diseases, resulting in immune hyporesponsiveness. Accordingly, the early treatment of AD with anti-inflammatory drugs at the MCI stage may be beneficial (429).

The antidiabetic drug rosiglitazone, which also has a neuroprotective effect, has been demonstrated to exert antiinflammatory effects by decreasing NF- $\kappa \mathrm{B}$ levels in neurons (231). Blunting autophagy/mitophagy leads to the generation of ROS in mitochondria, which, in turn, activates the NLRP3 inflammasome (474). Inflammasome activation contributes to the onset and progression of AD. The NLRP3 inflammasome was first identified in AD by studying the IL- $1 \beta$ expression induced by $\mathrm{A} \beta$ (148). In addition, studies crossing $N L R P^{-1-}$ mice with APP/PS1 mice demonstrated the role of the NLRP3 inflammasome in the pathogenesis of AD (155). Peripheral insulin resistance exacerbates brain inflammation, accelerating the Alzheimer-like pathology in $\mathrm{APP}^{+}$-ob/ob mice obtained from a cross of Alzheimer transgenic APP23 mice and diabetic ob/ob mice (384). Activating GSK3 $\beta$, a Tau phosphorylation kinase, could also induce inflammation via $\mathrm{NF}-\kappa \mathrm{B}$ and lead to apoptosis $(62,277)$.

The RAGE acts as an inflammatory mediator as well as an oxidative stress inducer in AD. RAGE belongs to the immunoglobulin superfamily and drives AD progression by mediating $\mathrm{A} \beta$ clearance, formation, and accumulation; NFT accumulation and neuronal degeneration. RAGE transports $\mathrm{A} \beta$ from the periphery to the brain, and transportation at the $\mathrm{BBB}$ disrupts $\mathrm{BBB}$ function (412). The RAGE-A $\beta$ interaction inhibits the nuclear translocation of NF- $\kappa \mathrm{B}$ and stimulates the release of proinflammatory cytokines, such as TNF- $\alpha$ and IL- 6 and ROS. The interaction between RAGE and $\mathrm{A} \beta$ maintains the balance of $\mathrm{A} \beta$ via the regulation of $\beta$ and $\gamma$-secretase activities. Changes in fatty acid composition were present in $\mathrm{AD}$ human cases, with an increase in RAGE leading to a lipid peroxidation-derived protein modification in $\mathrm{AD}$ pathogenesis (300).

Mitochondrial uncoupling proteins (UCPs) located on the inner mitochondrial membrane function as regulators of mitochondrial membrane potential and ROS, thus protecting neurons. Studies have indicated that UCP2 is a novel mast cell function regulator with potential for the treatment of mast cell-mediated allergic and inflammation responses in neurodegenerative diseases (197).

Indeed, mitochondrial dysfunction induces inflammation and oxidative stress; in contrast, inflammation and oxidative stress induce mitochondrial dysfunction. Either way, inflammation, oxidative stress, and mitochondrial dysfunction coexist and exacerbate each other; therefore, approaches targeting mitochondrial dysfunction, inflammation, oxidative stress, or any two or all of these events could be beneficial for $\mathrm{AD}$ prevention and treatment.

\section{E. Mitochondrial metabolic disorder links nutritional imbalance to neural DNA epigenetic remodeling}

The phenomenon of epigenetics has shown a new link between metabolic dysfunction and AD. Epigenetic changes, such as DNA methylation, have been observed in both metabolic disorders and AD. Metabolic disorders alter genome-wide
DNA hydroxymethylation in two ways in association with mitochondrial dysfunction: (i) decreasing the stability of the TET2 protein by impeding AMPK phosphorylation and (ii) inhibiting ten-eleven translocation (TET) activity by increasing $\mathrm{KGDH}$ to competitively inhibit fumarate and succinate.

5-Hydroxymethylcytosine $(5 \mathrm{hmC})$ is a major and stable epigenetic marker generated from 5-methylcytosine $(5 \mathrm{mC})$ by the TET family of dioxygenases that is involved in a wide range of biological processes from development to various diseases (53). The epigenetic modulation that mtDNA methylation $5 \mathrm{mC}$ was found in the D-loop region of mtDNA in the entorhinal cortex in the human AD brain supported the epigenetic mtDNA regulation in AD human cases (30). $5 \mathrm{hmC}$ is regulated by multiple factors, and TETs play a central role in the generation and maintenance of $5 \mathrm{hmC}$ (208). TET-mediated oxidation reactions require oxygen and $\mathrm{KGDH}$ as substrates and $\mathrm{Fe}$ (II) as a cofactor to generate $\mathrm{CO}_{2}$ and succinate. As a result, $5 \mathrm{hmC}$ is directly affected by the availability of the substrates and cofactors (115).

Mitochondria are the regulatory centers of iron. The iron that is not used inside the cell is stored within the ferritin macromolecule to avoid the exertion of free iron toxicity in the cytosol (35). Multiple studies have shown that vitamin $\mathrm{C}$ can improve TET activity by improving the Fe (II) level (200). Therefore, iron homeostasis disorder induced by mitochondrial dysfunction can directly affect the $5 \mathrm{hmC}$ level in the genome. KGDH, as an intermediate in the TCA cycle for energy metabolism, is generated from isocitratevia isocitrate dehydrogenases, including IDH1, IDH2, and IDH3. Mutations in IDH1/IDH2 result in the simultaneous loss and gain of activities in the production of $\mathrm{KGDH}$ and 2hydroxyglutarate $(2-\mathrm{HG})$, respectively (208). These findings provide a biochemical basis for the hypermethylation observed in human glioma with IDH mutations and the mutually exclusive manner of IDH1/IDH2 and TET2 gene mutations in acute myeloid leukemia. In addition to IDH, two other TCA cycle genes, fumarate hydratase and SDH, are mutated in numerous human cancers, leading to the accumulation of fumarate and succinate. More importantly, 2-HG, fumarate, and succinate, which act as antagonists of $\mathrm{KGDH}$, competitively inhibit the activity of TETs and the consequent alterations in genome-wide DNA hydroxymethylation $(13,143,176,436)$.

In addition to gene mutations, AMPK is impeded by high glucose levels, which results in the destabilization of TET2, followed by the dysregulation of $5 \mathrm{hmC}$ (135). Our recent work shows that the dysregulation of $5 \mathrm{hmC}$ in the CNS of mice with metabolic disorders induced by an HFD is closely related to the decreased stability of the TET2 protein and that the increased contents of TET enzyme inhibitors (fumarate and succinate) are induced by an HFD. Notably, knockdown of the TET2 gene leads to mitochondrial dysfunction and inflammation (unpublished) (Fig. 6).

\section{Mitochondria-Centered Metabolic Markers in Early AD}

There is an urgent need to identify alternative disease mechanisms and associated biomarkers that can help to diagnose $\mathrm{AD}$ in the preclinical and early clinical stages. Given that central and peripheral metabolism are remodeled in the early stage of $\mathrm{AD}$ and that mitochondria play a central role in the pathogenesis of $\mathrm{AD}$, mitochondrial biomarkers 


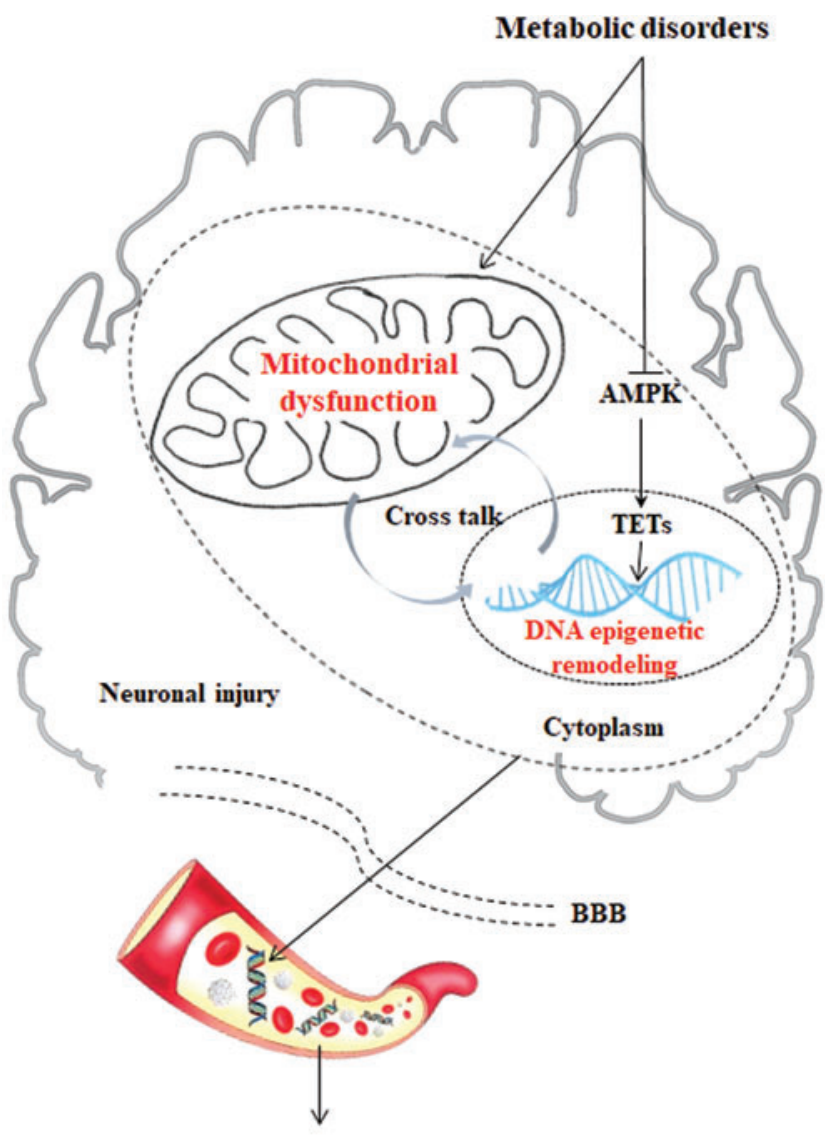

Circulating cell-free DNA epigenetic modifications

FIG. 6. Circulating cfDNA epigenetic modifications from brain metabolic disorder and mitochondrial dysfunction. Metabolic disorders induce $5 \mathrm{hmC}$ dysregulation in two ways. Metabolic disorders in the TCA cycle may accumulate fumarate and succinate, the competitive inhibitors of $\alpha$-ketoglutarate-dependent dioxygenases, leading to enzymatic inhibition of TETs and consequent alterations of genome-wide DNA epigenetic modifications. Metabolic disorders inhibit AMPK phosphorylation, resulting in the destabilization of TET2, followed by the dysregulation of $5 \mathrm{hmC}$, which could activate neuronal injury. cfDNA is released from cells undergoing apoptosis, necrosis, and secretion, and it can be shed from the brain to the bloodstream. Therefore, we hypothesize that the proportion of the cfDNA in plasma originating from the synapses and neurons will indicate the $\mathrm{AD}$ pathogenesis, which provides us with a greater opportunity to identify a novel $5 \mathrm{hmC}$ marker in cfDNA during $\mathrm{AD}$ pathological progression. 5hmC, 5hydroxymethylcytosine; cfDNA, cell-free DNA; TET, teneleven translocation. Color images are available online.

in the CNS and periphery could be targeted for the early diagnosis of $\mathrm{AD}$.

\section{A. Biomarkers in the brain and CSF}

Another characteristic feature of $\mathrm{AD}$ is a reduced rate of brain metabolism, occurring before the development of significant amyloid plaques and NFTs $(31,166)$. The mitochondrial proteome in $\mathrm{AD}$ was analyzed by quantitative comparative proteomic profiling (59). Studies of transgenic $\mathrm{AD}$ mice have demonstrated that a wide variety of metabolic deficits, including the citric acid cycle, OXPHOS, pyruvate metabolism, glycolysis, oxidative stress, fatty acid oxidation, ketone body metabolism, ion transport, apoptosis, and mitochondrial protein synthesis, occur early in AD (99). COX (complex IV) of the electron transport chain shows decreased activity in both AD brains and platelets (124), as does the activity of the PDC, which connects glycolysis to the citric acid cycle in AD cortices $(59,250)$. Further, numerous mitochondrial proteins are reduced in AD cortices, including the subunits of complex I and complex IV in OXPHOS (59). Alternatively, other mitochondrial proteins, including malate dehydrogenase and SDH, show increased activity in $\mathrm{AD}$ cortices (447).

Moreover, some mitochondrial enzymes involved in energy production, such as acetyl-CoA dehydrogenase and enoyl-CoA hydratase (ECHS1), were upregulated in $\mathrm{AD}$ cortices (59). Increased levels of ketone body-producing enzymes have been previously observed in $\mathrm{AD}$ mouse models. The expression of 3-oxoacid-CoA transferase 1, which catabolizes ketone bodies to produce acetyl-CoA and thus generate ATP, was increased in $3 \times \mathrm{TgAD}$ mice (447). Protein-methionine sulfoxide oxidase is the enzymatic source of ROS production in neurons, and it could act as an increased biomarker in the AD brain (34). The removal of cholesterol from the brain is controlled by cytochrome $\mathrm{P} 450$ and was found to be genetically enhanced in $5 \times \mathrm{FAD}$ mice $(126,268)$. These alterations in the mitochondrial proteome of $\mathrm{AD}$ mouse cerebral cortices indicate that central mitochondrial protein alterations might be possible biomarkers for the early diagnosis of $\mathrm{AD}$.

Metabolomics is a powerful tool for studying perturbations in the metabolome, reflecting alterations in multiple networks affected in AD. Previous studies have shown that many canonical pathways are significantly disturbed in $\mathrm{MCI}$ and $\mathrm{AD}$ patients. These pathways include energy metabolism, the Krebs cycle, mitochondrial function, neurotransmitters, amino acid metabolism, and lipid biosynthesis. Krebs cycle markers in the CSF and liver were significantly affected in patients with MCI compared with unaffected people. Succinic anhydride, citraconic acid, 2-furoic acid, threo-isocitric acid, citraconic acid, and pyruvic acid were increased in the CSF of MCI patients; however, acetoacetic acid and fumaric acid levels were decreased. Interestingly, most of the mitochondria-related metabolites affected early in MCI continue to be altered in $\mathrm{AD}$, including succinic anhydride, citraconic acid, pyruvic acid, and acetoacetic acid (87). Moreover, F2-isoprostanes, which are stable products of lipid peroxidation, accumulate in the CSF of AD patients (126, 127). In addition, a new study reported reduced levels of cell-free mtDNA in the CSF, representing a possible novel biomarker of preclinical AD (83).

In 2018, the National Institute of Aging and Alzheimer's Association proposed a diagnostic standard for $\mathrm{AD}$ that is abbreviated as the ATN system (177), where A stands for $\beta$ amyloid deposition, $\mathrm{T}$ stands for pathologic tau including total tau and phosphorylated tau, and $\mathrm{N}$ stands for neurodegeneration. Based on long-term clinical studies, this ATN classification system clusters different biomarkers through neuroimaging techniques, such as PET, thus providing standardized indicators for basic and clinical researchers to distinguish the cognitive impairment caused by AD pathology, even at an early stage (30-50 years old), thereby achieving 
early intervention. Many new mitochondria-related changes have been found in the CSF of transgenic AD mice and patients and are thus indicated as potential biomarkers. These mitochondria-related biomarkers should be further verified for their specificity in AD.

PET testing is expensive, as it requires demanding equipment and strict radioisotope operation. Further, the collection of CSF samples requires lumbar puncture, which is highly invasive. In addition, the contents of recent standard AD biomarkers found in the CSF are very low in the blood. Therefore, it is necessary to use peripheral body fluids, especially blood, to detect new biomarkers for the early diagnosis of AD.

\section{B. Biomarkers in peripheral tissues and circulating body fluids}

$\mathrm{AD}$ is a multifactorial disease that affects both the CNS and the periphery (127). Intriguingly, several recent studies investigating metabolites have reported significant metabolic changes in the liver and other peripheral organs $(23,325)$. $\beta$-oxidation, in liver mitochondria or peroxisomes (325), is the principal pathway for fatty acid metabolism. AD-related increases in long-chain specific acyl-CoA dehydrogenase, 3-keto-CoA thiolase, and acyl-CoA thioesterase 10 have been observed, suggesting elevated fatty acid $\beta$-oxidation and increased acetyl-CoA production (129). The increased fatty acid metabolism in the AD mouse liver may increase ketone body formation in blood, supported by the augmented ketone body production in AD mice (136). Pyruvate is an essential metabolite that fuels TCA and drives other biosynthetic pathways (87).

Other studies have shown that the levels of hydroxymethyl transferase and NADP-dependent malic enzyme are increased in the AD liver, while serine-pyruvate amino transferase levels are decreased, suggesting elevated pyruvate concentrations (129). Further, the decreased expression of glycogen synthase and glycogen breakdown proteins in the AD liver suggests reduced glucose storage (83). The observed proteomic changes suggest dysregulated glucose in the AD mouse liver (129). The enzymes involved in the electron transport chain can generate ROS by forming superoxide anions from molecular oxygen (396). COX-2 and sulfite oxidase were observed in the AD liver and thus potentially increase OXPHOS (129). Alterations in antioxidant regeneration rely on a continuous supply of NADH and NADPH and have been reported in the AD liver (136). The redox state in mitochondria depends on the ratios of the reduced and oxidized forms of several systems such as $\mathrm{NADH} / \mathrm{NAD}^{+}$(401). The changes observed in the liver proteome of $\mathrm{AD}$ mice provide further evidence that $\mathrm{AD}$ is a metabolic disorder and that the metabolites produced in mitochondrial metabolism might be liver biomarkers for early diagnosis and prevention.

Mitochondrial dysfunction has been demonstrated in the neurons of AD patients (396) and further shown to be linked to alterations in ROS production (462). These changes were observed in the brains of AD patients as well as in the periphery, that is, fibroblasts in humans and in mouse models $(396,462)$, and other tissues, including platelets and lymphocytes. Several studies showed that lipid oxidation and superoxide dismutase (SOD) activity were increased; whereas the activities of complex IV, complex III, mitochondrial membrane potential, and ATP were reduced in the platelets of AD patients compared with age-matched controls $(167,256$, 275, 287).

Further, increased levels of oxidative DNA damage, Mn-superoxide-dismutase (SOD2) mRNA, 4-HNE, and 3nitrotyrosine are observed in the lymphocytes of AD patients $(358,397)$, and ROS levels are higher in the lymphocytes of both $\mathrm{AD}$ patients and transgenic $\mathrm{AD}$ animals $(74,375)$. Moreover, apoptosis levels are elevated in lymphocytes from AD patients and transgenic mice as indicated by enhanced DNA fragmentation, SOD mRNA expression, $\mathrm{Bax} / \mathrm{Bcl} 2$ ratios and caspase-3, caspase-6, and caspase-8 activity $(93,120,220,382)$.

In addition, Scott et al. reported that basal mitochondrial oxygen consumption, proton leakage, and AP endonuclease 1 (APE1) activity were reduced in peripheral blood mononuclear cells; whereas dATP levels were enhanced. MtDNA depletion is believed to be a typical pathophysiological factor of neurodegeneration in AD. A low cell-free mtDNA content in CSF may be a novel biomarker for the early detection of preclinical AD in both patients and transgenic mice. Further studies are needed to determine whether the CSF mtDNA concentration can be altered by disease-modifying treatments (315). Our laboratory recently identified cell-free DNA 5hmC as a potential biomarker in $\mathrm{AD}$ (unpublished). These studies highlight the relevance of mitochondrial function in blood cells as an early peripheral marker for the detection of AD.

Alterations in several types of mitochondrial enzymes and metabolites have recently been reported; however, selecting reliable and usable biomarkers from a large number of mitochondrial-related proteins is still a major challenge for the diagnosis, prevention, and control of AD. Detecting biomarkers in blood is the better method for diagnosing AD, but this has not yet been achieved. The identification of sensitive and easily detectable biomarkers should be a major goal in AD diagnosis, and the development of early diagnostic techniques has also advanced the study of early AD intervention programs (Fig. 7).

\section{Targeting Mitochondrial Metabolic Dysfunction for AD Prevention}

There are currently more than 2000 registered clinical trials worldwide investigating anti-AD drugs that can be classified by their main mechanisms, including (i) targeting neurotransmission, (ii) preventing the accumulation of amyloid plaques or NFTs, and (iii) restoring mitochondrial function or energy metabolism; and other therapeutic approaches, such as anti-inflammation or rescuing nerve growth factor (ClinicalTrials.gov). Currently, the main therapeutic strategies and corresponding drug research and development include the following: (i) drugs that interfere with the formation and deposition of $\mathrm{A} \beta$ and amyloid cascade reaction; (ii) drugs that affect aggregated hyperphosphorylated Tau proteins; and (iii) drugs that regulate the CNS, including different types of neuronal receptors involved in neuronal plasticity and signal transduction.

The clinically approved drugs for AD treatment include (i) donepezil, an acetylcholinesterase inhibitor for all stages of $\mathrm{AD}$; (ii) rivastigmine, an acetylcholinesterase inhibitor and butyryl cholinesterase inhibitor for mild to moderate AD; (iii) galantamine, a nicotinic receptor modulator for mild to moderate AD; (iv) memantine, an N-methyl D-aspartate (NMDA) 


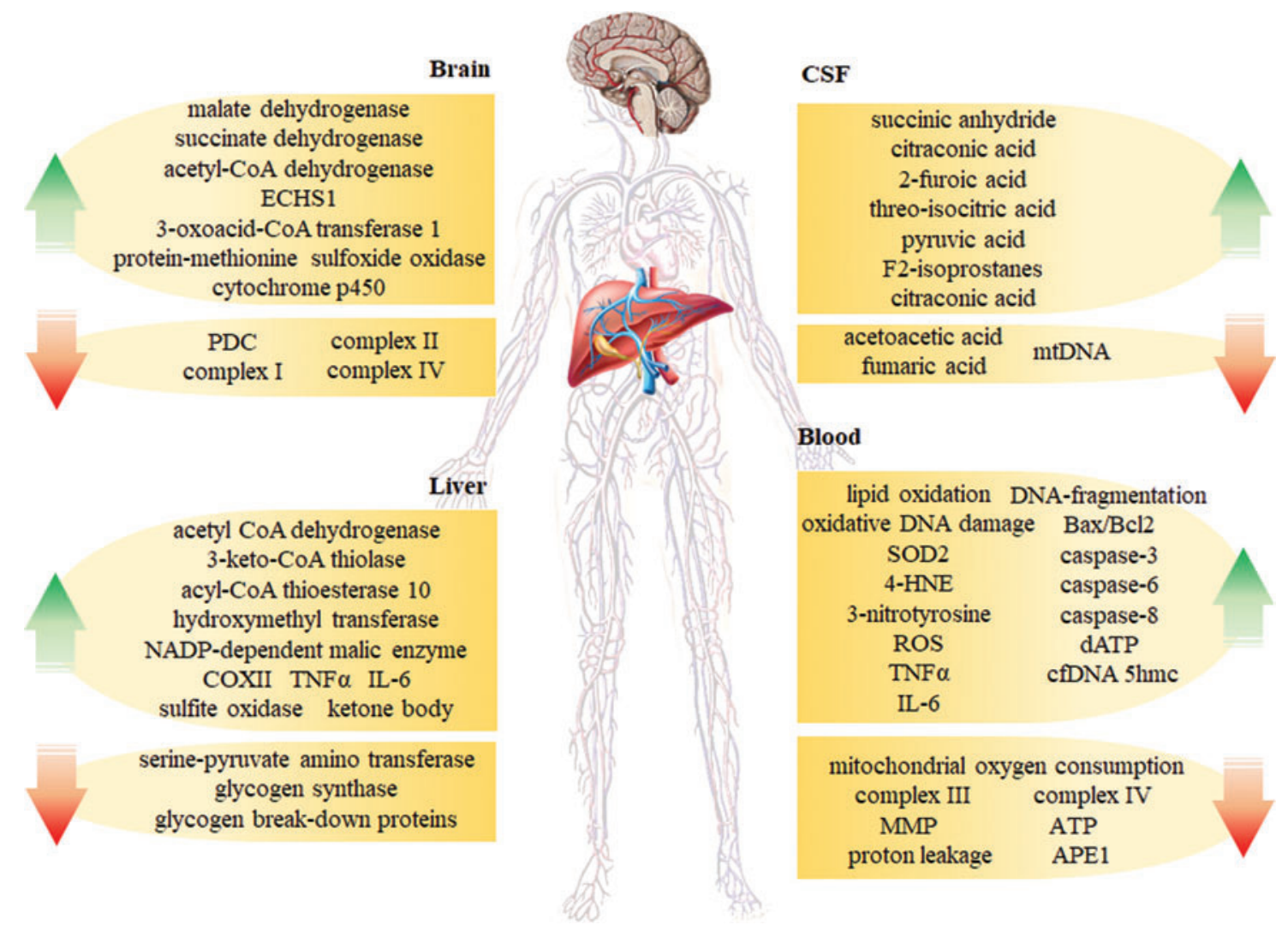

FIG. 7. Mitochondria-centered biomarkers in the AD brain, liver, CSF, and blood. As mitochondria play a central role in $\mathrm{AD}$ pathogenesis, mitochondria-centered biomarkers in the CNS and periphery could be new candidates for the early diagnosis of AD. Alterations in several types of mitochondrial enzymes and metabolites in the brain, liver, CSF, and blood are shown. Detecting biomarkers in blood is the better method for diagnosing AD, but this has not yet been achieved. The identification of sensitive and easily detectable biomarkers should be a major goal in AD diagnosis, and the development of early diagnostic techniques has also advanced the study of early AD intervention programs. The up arrows indicate the biomarkers that are upregulated in $\mathrm{AD}$, whereas the down arrows indicate the biomarkers that are downregulated in $\mathrm{AD}$. CSF, cerebrospinal fluid; ECHS1, enoyl-CoA hydratase; MMP, mitochondrial membrane potential; PDC, pyruvate dehydrogenase complex; ROS, reactive oxygen species; SOD2, Mn-superoxide-dismutase. Color images are available online.

receptor antagonist for moderate to severe $\mathrm{AD}$; and (v) huperzine A, an acetylcholinesterase inhibitor approved in China for mild to moderate AD that could also be used as an antioxidant to protect mitochondria. These drugs ameliorate AD-related symptoms in the short term; however, there are no drugs currently available for inhibiting AD progression (261). The unique bioenergetics alterations show promise as a method for preventing or delaying (or both) the onset of AD. Nutraceutical interventions that improve metabolic activity and mitochondrial function could be a candidate approach to preventing AD.

\section{A. Mitochondrial nutrients}

"Mitochondrial nutrients" refer to a group of micronutrients that, either directly or through metabolites, influence the structure and function of mitochondria $(234,239)$. These nutrients may also have other functions that are not necessarily related to mitochondria. The nutrients benefit mitochondria in four ways: by (i) ameliorating oxidative stress, for example, lipoic acid and hydoxytyrosol $(234,307)$; (ii) activating phase II enzymes that improve antioxidant defenses, for example, tocopherol and sulforaphane (106, 116); (iii) enhancing mitochondrial remodeling, including mitochondrial degradation, biogenesis, fission, and fusion, for example, acetyl-L-carnitine (ALCAR) and hydroxytyrosol acetate (HT-ac) $(237,418)$; and (iv) protecting mitochondrial enzymes and/or stimulating mitochondrial enzyme activities, for example, enzyme cofactors, such as B vitamins and coenzyme $\mathrm{Q}(234,237)$. In addition to using mitochondrial nutrients individually, the combined use of mitochondrial nutrients may provide a better strategy for mitochondrial protection $(149,237)$ (Fig. 8). A table is compiled based on the clinical data registered on the ClinicalTrials.gov database, which is maintained by NLM at the NIH, to do a proper analysis for mitochondrial nutrients in $\mathrm{AD}$ (Table 1). The detailed information of the trial can be referred to the Identifier listed in the table.

1. Lipoic acid. $\alpha$-Lipoic acid (LA), the in vivo cofactor for the PDC and KGDH in the TCA cycle, inhibits the formation of ROS, increases the levels of reduced glutathione, and scavenges lipid peroxidation products, such as hydroxynonenal and acrolein. LA crosses the BBB into nerve cells and protects against cell damage induced by $\mathrm{A} \beta$ or hydrogen peroxide $(77,235,442)$. It also induces Akt expression, suggesting that the neuroprotective effect of the antioxidant 


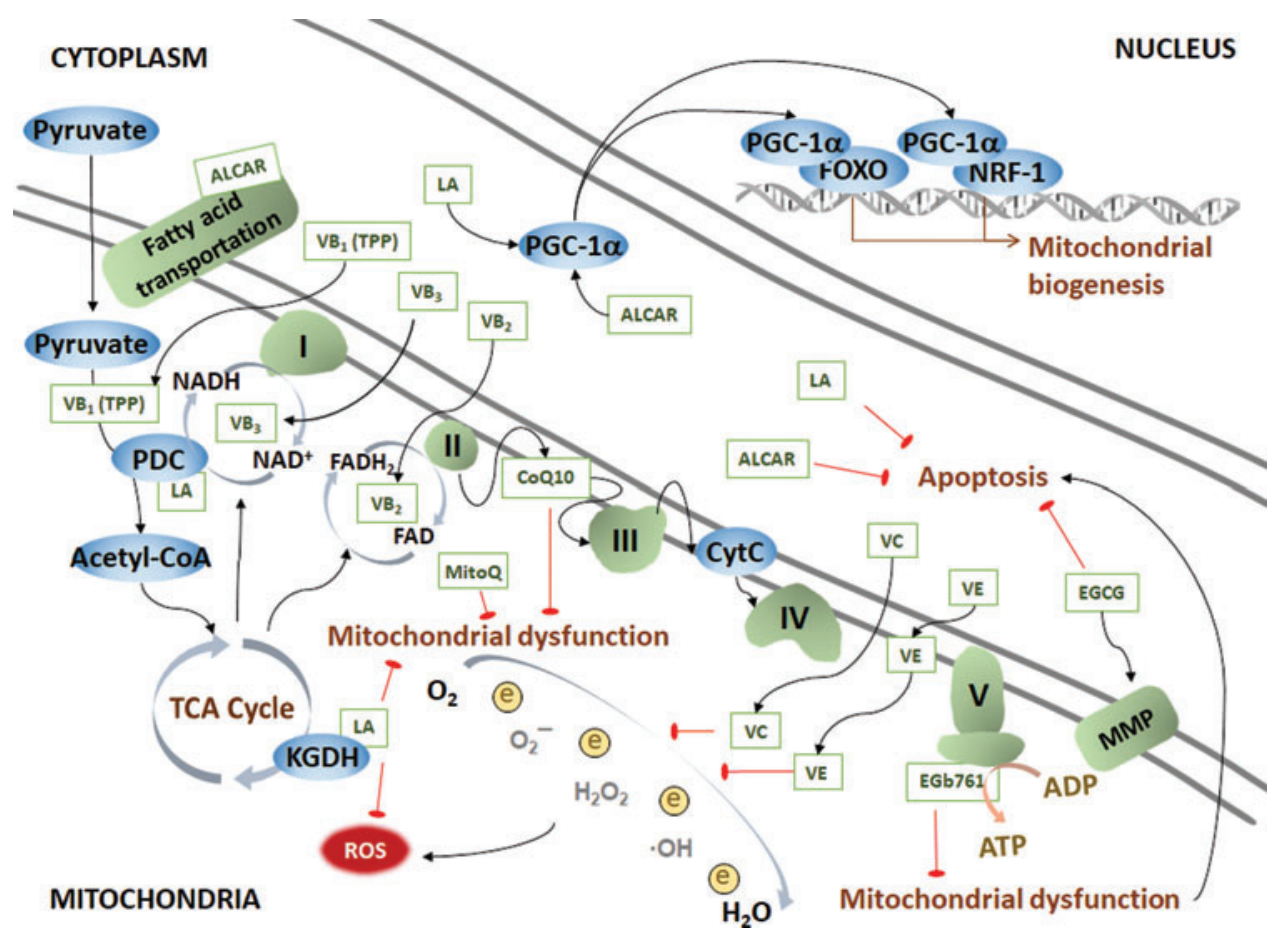

FIG. 8. Functions of LA, ALCAR, CoQ 10 , vitamins B, C, and E, EGCG, and EGb761 for AD. LA is the cofactor for PDC and KGDH in the TCA cycle and inhibits the formation of ROS. Other protective mechanisms for LA include inhibiting apoptosis. In addition, LA targets PGC- $1 \alpha$, which binds to FOXO or NRF1 to increase mitochondrial biogenesis. ALCAR transports and activates fatty acids into the mitochondria, protects nerve cells from apoptosis, and target, PGC-1 $\alpha /$ FOXO or PGC-1 $\alpha / \mathrm{NRF} 1$ signaling to improve mitochondrial biogenesis. $\mathrm{COQ}_{10}$ is an integral part of the electron transport chain. The role of $\mathrm{CoQ}_{10}$ in $\mathrm{AD}$ is to stabilize mitochondria. MitoQ is a modified $\mathrm{CoQ}_{10}$ molecule that is more easily absorbed and attracted to mitochondria, thereby improving electron transport chain function. B vitamins are components or precursors of corresponding mitochondrial coenzymes. The active form of vitamin $\mathrm{B}_{1}$ is TPP. Vitamin $\mathrm{B}_{2}$ participates in the transformation of $\mathrm{FADH}_{2}-\mathrm{FAD}$, and vitamin $\mathrm{B}_{3}$ participates in the transformation of NADH-NAD ${ }^{+}$. Vitamins C and $\mathrm{E}$ scavenge free radicals through oxidative stress pathways. EGb761 reverses the decrease in ATP production, thereby ameliorating mitochondrial dysfunction. EGCG intervention effectively protects against mitochondrial dysfunction through increasing MMP and protects against apoptosis. ALCAR, acetyl-L-carnitine; CoQ10, coenzyme Q10; EGCG, epigallocatechin-3-gallate; KGDH, $\alpha$-ketoglutarate dehydrogenase complex; LA, $\alpha$-lipoic acid; TPP, thiamine pyrophosphate. Color images are available online.

LA is partially transmitted through PKB/Akt signaling. LA also exerts protective effects by activating phase 2 enzymes specifically by inducing the expression of Nrf2, which binds to ARE, in turn increasing the activity of the phase 2 enzyme system to reduce cellular oxidative stress, enhancing the intracellular antioxidant defense system, and alleviating mitochondrial oxidative stress. On the other hand, LA targets PGC- $1 \alpha$, which binds to FOXO or NRF1 to increase mitochondrial biogenesis, thereby improving cognitive function in $\mathrm{AD}(145,374)$. A supplementation of LA and N-acetyl cysteine decreased mitochondrialrelated oxidative damage in AD patient fibroblasts (275). A 48-month follow-up study in AD patients showed that treatment with LA might be a neuroprotective therapeutic option for AD. However, the phase II trial is needed (145).

2. Acetyl-L-carnitine. ALCAR is a natural product of the reaction of carnitine with acetyl-CoA. ALCAR is used to transport and activate fatty acids into the mitochondria, which is a rate-limiting regulator of fatty acid oxidation. ALCAR protects nerve cells from apoptosis and targets PGC$1 \alpha /$ FOXO or PGC- $1 \alpha /$ NRF 1 signaling to improve mitochondrial biogenesis. ALCAR is especially enriched in muscle and brain tissues and can efficiently travel across the BBB to promote nerve cell regeneration, protect nerve cells from mitochondrial uncouplers and inhibitors, and enhance mitochondrial enzyme activity and mitochondrial function, thereby reducing brain damage in $\operatorname{AD}(144,235,341)$. After 6 months of the double-blind parallel design pilot study of ALCAR, the ALCAR group demonstrated significantly slowed deterioration in some cognitive areas among patients with $\mathrm{AD}$ (341). The perfusion of the precuneus was amplified in AD patients after ALCAR administration and their cognitive and neuropsychiatric symptoms were not worsened (180). Further clinical studies are warranted to determine the role of ALCAR treatment in AD.

3. Coenzyme $\mathrm{Q}_{10}$ and MitoQ. Coenzyme $\mathrm{Q}_{10}\left(\mathrm{CoQ}_{10}\right)$ is an integral part of the electron transport chain that acts as an electron acceptor that promotes ATP production and as an antioxidant in the mitochondrial matrix and its inner membrane. Several studies have identified $\mathrm{CoQ}_{10}$ as a potential target for $\mathrm{AD}$, as stabilized mitochondria impaired by neurotoxins and oxidative stress and improved memory and behavioral performance in Tg19959 and APP/PS1 mouse 


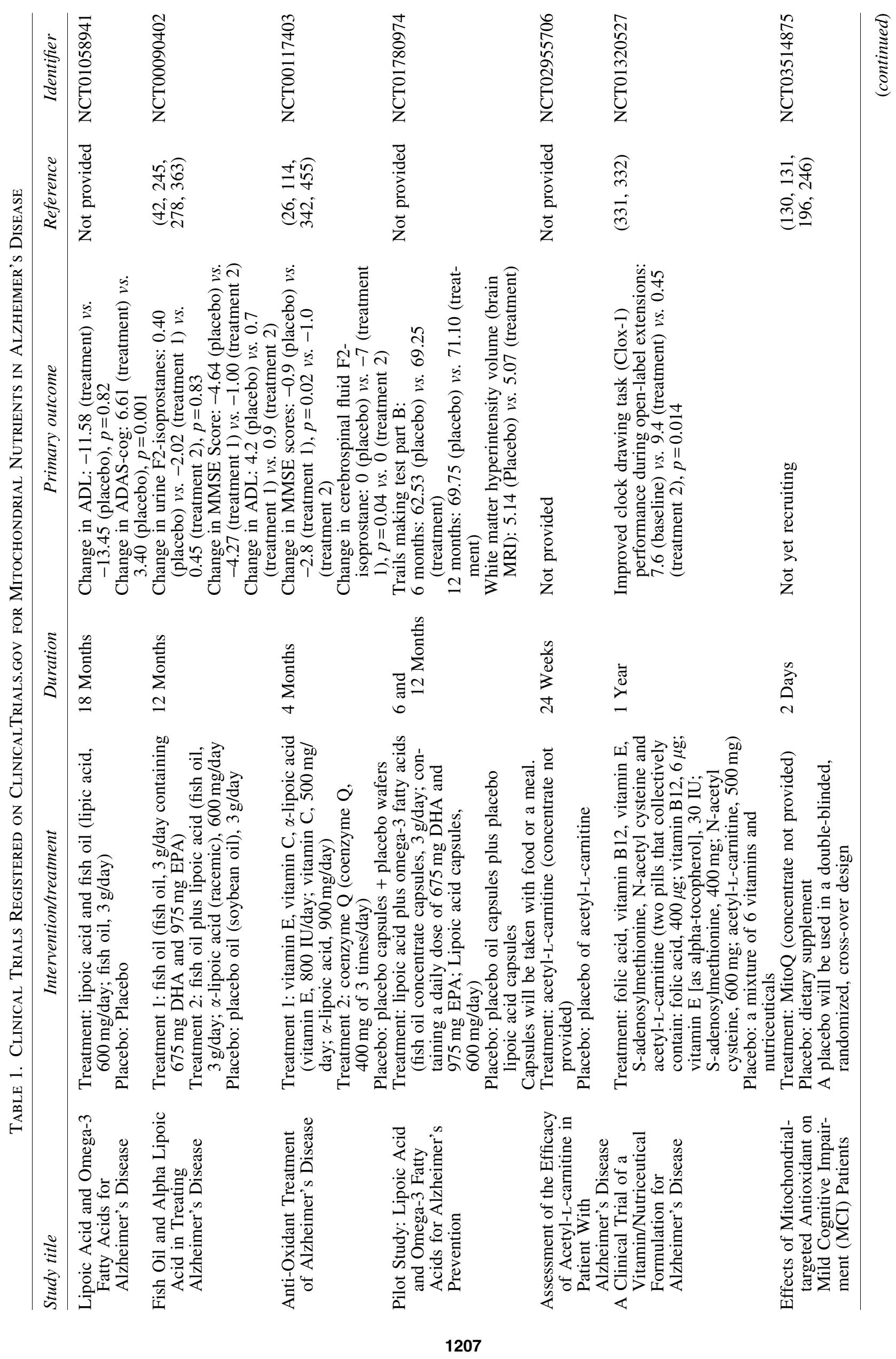




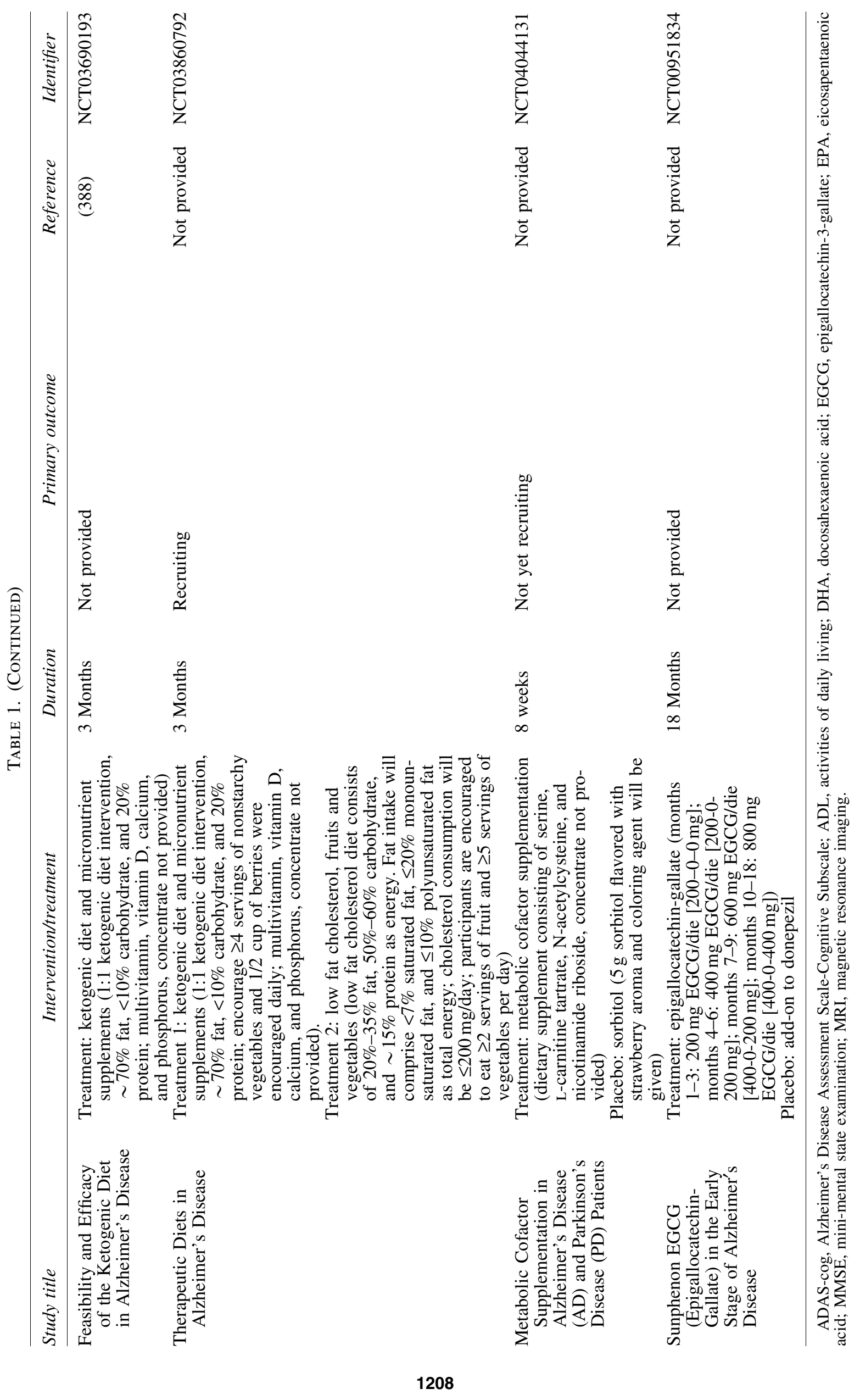


models of $\mathrm{AD}(88,286)$. The levels of the reduced and oxidized forms of $\mathrm{CoQ}_{10}$ in the CSF of $\mathrm{AD}$ patients and agematched controls measured using HPLC showed that the oxidized/total $\mathrm{CoQ}_{10}$ level in $\mathrm{AD}$ was significantly higher than those in the controls, indicating that mitochondrial oxidative damage plays an important role in early AD pathogenesis (175). The mitochondria-targeting antioxidant MitoQ is a modified $\mathrm{CoQ}_{10}$ molecule. Compared with $\mathrm{CoQ}_{10}$, MitoQ is a much smaller molecule that is more easily absorbed by cells and is attracted to negatively charged mitochondria. MitoQ treatment was shown to prevent cognitive decline and oxidative stress in $3 \times \operatorname{TgAD}$ mice $(269,453)$ and to extend the lifespan, improve electron transport chain function, and protect the mitochondrial cardiolipin content in a Caenorhabditis elegans model of AD (292).

4. B vitamins. B vitamins have been found to reduce brain atrophy and slow the onset or progression of $\mathrm{AD}(82)$. B vitamins are components or precursors of corresponding mitochondrial coenzymes and participate in one-carbon metabolism $(86,395)$. Thiamine pyrophosphate is an active form of vitamin $B_{1}$ that is responsible for glucose metabolism and the eventual production of acetyl-CoA. Vitamin $\mathrm{B}_{2}$ (riboflavin) is the precursor of FAD that participates in the transformation of $\mathrm{FADH}_{2}-\mathrm{FAD}$ in energy production and cellular respiration. Vitamin $\mathrm{B}_{3}$ is the precursor for NAD and NADP, which are responsible for DNA repair and mitochondrial respiration. However, vitamin B supplementation for $\mathrm{AD}$ remains controversial, because some trials failed to show its protective effect.

5. Hydroxytyrosol. Hydroxytyrosol (HT) is a phenyl ethanol with antioxidant activities. HT could supply a hydrogen atom from the phenolic hydroxyl group to free radicals. The second hydroxyl group in the ortho-position enhances antioxidation by generating the catechol ring, thereby increasing the rate of hydrogen transfer to the superoxide radical. The catechol ring is partially converted into o-benzoquinone and plays a direct role as an antioxidant.

There are two main sources of HT: (i) exogenous HT (oleuropein undergoes a double hydrolysis reaction to form HT, which is mainly found in olives, olive leaves, olive pulp, and olive oil in the Mediterranean diet); (ii) endogenous HT, the dopamine oxidation metabolite in the body. HT has now been identified by the Scientific Committee of the European Food Safety Authority as a polyphenol in olive oil that can help to protect lipids from oxidation. The pharmacokinetics of HT have been extensively studied, and one 14C-labeled HT distribution study showed that HT could exert its neuroprotective effect across the BBB (206). The cardioprotective, antitumor, antimicrobial, antidiabetic, and neuroprotective effects of HT have been investigated, due to its high antioxidant activity (337). Two major mechanisms involved in the regulation of HT antioxidant activity are: (i) the direct removal of ROS generated by oxidative stress and (ii) the activation of certain molecular targets to resist oxidative stress (Fig. 9).

Adherence to the Mediterranean diet could reduce the risk of neurodegenerative diseases, such as $\mathrm{MCI}$ and $\mathrm{AD}$, while helping to improve cognitive function $(316,335,346,366)$. The $2019 \mathrm{WHO}$ guidelines for reducing the risk of cognitive decline and dementia recommended a Mediterranean-like diet to adults with normal cognition and MCI to reduce the risk of cognitive decline and/or dementia (425). One possible reason for the beneficial effects of the Mediterranean diet might be the functional ingredients, such as HT, which is the beneficial component of olive products. However, there are very few studies on the neuroprotective effect of HT. Oleuropein supplementation in TgCRND8 mice and aged mice resulted in a significant improvement in cognitive function; in addition, $\mathrm{A} \beta$ levels and plaque deposition in the brain were significantly reduced. These changes may be
FIG. 9. Functions of HT in AD. HT removes mitochondrial ROS and activates certain molecular targets. For example, HT activates $\mathrm{JNK} / \mathrm{p} 38-\mathrm{NF}-\kappa \mathrm{B}$ signaling to release proinflammatory interleukins and cytokines; improves the JAK2/STAT3, ERK/ RSK2, and AMPK/AKT signaling pathway for neuronal survival; repairs the phase 2 enzyme system by activating Nrf2/Keap1 signaling; and activates AMPK/ PGC $-1 \alpha$ to trigger mitochondrial biogenesis. HT, hydroxytyrosol; JNK, C-Jun NH2-terminal kinase. Color images are available online.

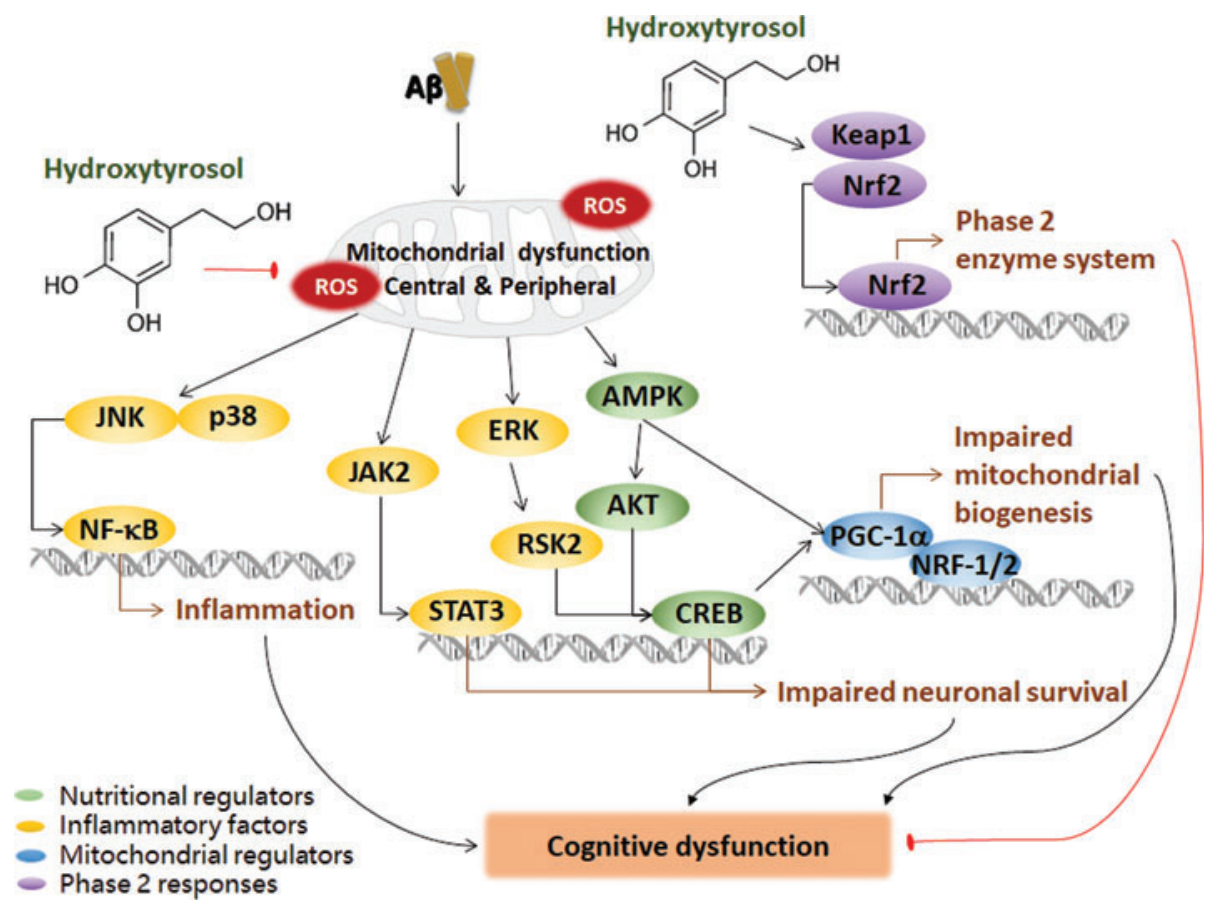


achieved through the mTOR pathway and, subsequently, activate neuronal autophagy (133). Another study showed that 14 days of $10 \mathrm{mg} / \mathrm{kg}$ HT treatment significantly enhanced the spatial cognitive abilities of C57BL/6 mice. The animals were injected with oligomeric $\mathrm{A} \beta_{1-42}$ plus isonicotinic acid and the improved spatial ability may have been due to the HT-induced improvements in the ERK-MAPK/RSK2, PI3K/ Akt1, and JAK2/STAT3 signaling pathways in hippocampal neurons (16).

We identified mitochondrial dysfunction in AD and found that HT successfully reversed the mitochondrial respiratory chain enzyme activities in the brains of AD mice (307). A $\mathrm{GSH}$ reduction induces redox dysregulation and loss of mitochondrial membrane potential, and HT increases GSH levels via activating Nrf2. We also showed that HT significantly reduces mitochondrial protein carbonylation and repairs the phase 2 enzyme system by activating the Nrf2Keap1 pathway and AMPK to constitute the energy-sensing protein network and trigger mitochondrial biogenesis (469, 476). Some vitagenes respond to the phase 2 enzyme system to protect against electrophiles and oxidants, and the Keap1Nrf2/ARE pathway is critical for the phase 2 response. Vitagenes are protective genes that control stress and protein homeostasis with the health (40). Regulation of endogenous cellular defense mechanisms such as the vitagene network may open up new treatments for diseases related to tissue damage and cell death in AD.

Recent studies have reported that mitochondria may be a key target of HT in the amelioration of oxidative stress in AD. The oral administration of HT significantly improved the electroencephalogram activity of APP/PS1 mice as well as the learning and memory abilities and decreased mitochondrial oxidative stress, inflammation, and apoptosis in APP/ PS1 mice. Our group recently identified an HT derivative
HT-ac that has better bioavailability than HT and could remarkably improve cognitive function in APP/PS1 mice (unpublished). These findings suggest that HT may be a major functional factor in the Mediterranean diet to prevent and ameliorate AD (307) (Fig. 9).

6. $\beta$-hydroxybutyrate. Neuroprotective effects are observed with the appropriate concentrations of $\beta-\mathrm{HB}$, as it provides mitochondrial protection (461). $\beta$-HB reduces ROS levels by targeting complex I in the mitochondrial respiration chain and induces ATP production in brain mitochondria (252, 393). Medium-chain triglycerides, such as $\beta$-HB, might improve memory in $\mathrm{AD}$, which correlates positively with the plasma levels of $\beta$-HB. $\beta$-HB blocks $\mathrm{A} \beta$ entry and improves cognition in AD mice (72). Ketone bodies also have antiapoptotic activity by blocking ROS-activated PP2A, alleviating the inactivation of the antiapoptotic factor $\mathrm{Bcl} 2$ and thereby inhibiting apoptosis (251). In addition, $\beta$-HB may relieve rotenone-induced caspase 3 and caspase 9 activation (173). A recent study found that $\beta$-HB inhibited histone deacetylases (HDACs), releasing HDACs from closing the forkhead box $\mathrm{O} 3 \alpha$ and metallothionein 2 genes and triggering cellular antioxidant responses accordingly (291). The activated ketone body $\beta$-HB in the liver activates Bdnf promoters via HDAC2 and HDAC3, leading to neurotransmitter release (367).

$\beta$-HB has also been found to protect hippocampal neurons against $\mathrm{A} \beta$ toxicity in $\mathrm{A} \beta$-induced $\mathrm{AD}$ cell models by correcting defects in mitochondrial energy generation (193). These studies reveal that ketones, as an alternative energy source in the absence of glucose, may protect neurons by improving mitochondrial function, thereby relieving oxidative stress and inhibiting apoptosis. The detailed mechanisms by which $\beta$-HB and ketogenesis function in AD pathology are limited and worthy of further investigation (Fig. 10).

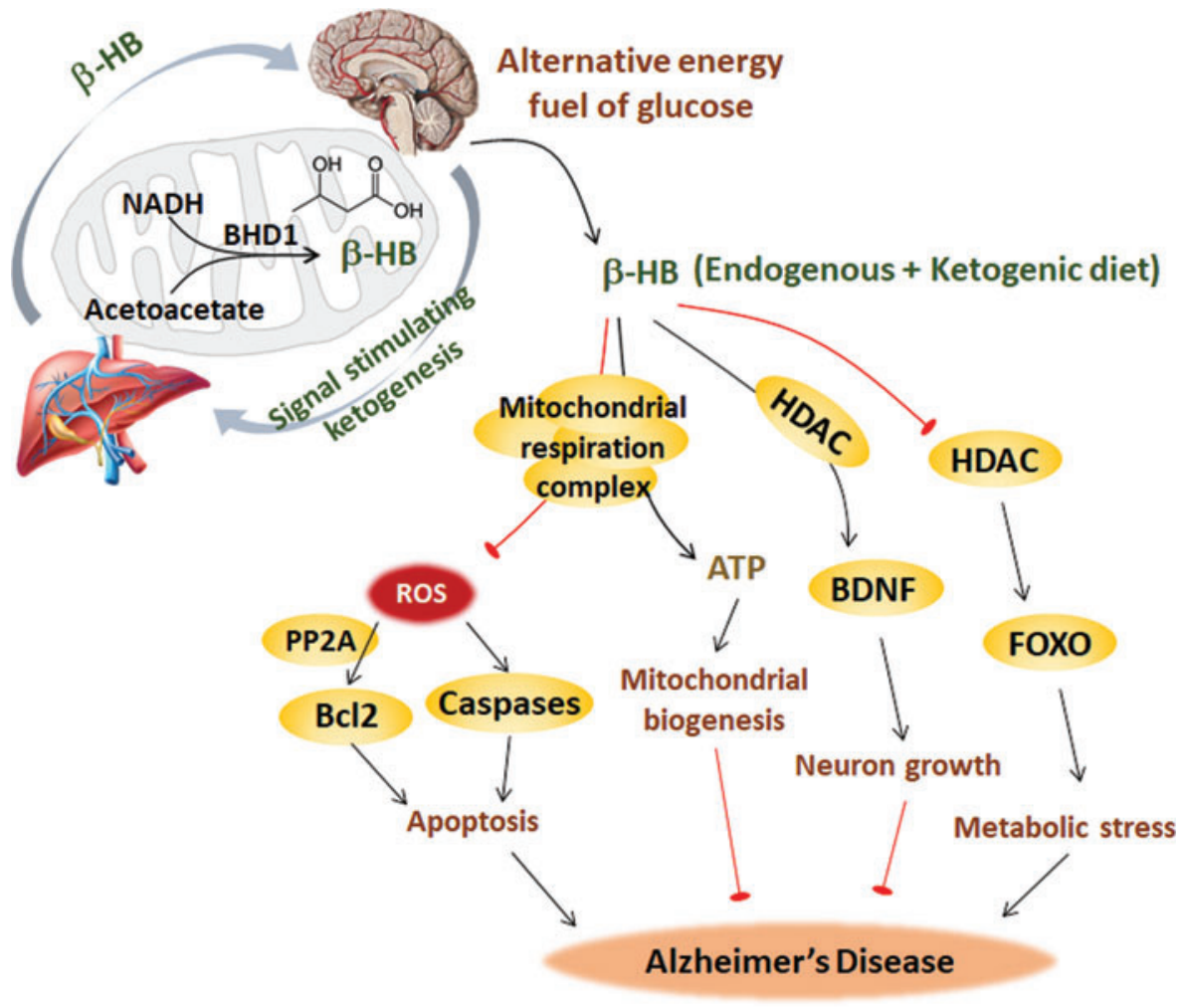

FIG. 10. Functions of $\beta-H B$ in AD. Ketogenesis, representative product as $\beta$-HB, is supposed to be stimulated by $\mathrm{AD}$ pathologyinvolved factor, and it is transferred across BBB as an alternative brain energy source. Memory improvement in AD correlates positively with the levels of $\beta$-HB. In addition, $\beta$-HB reduces ROS by targeting complexes in the mitochondrial respiration chain and induces ATP production to improve mitochondrial biogenesis. $\beta$-HB has antiapoptotic activity by inhibiting ROS-activated PP2A to alleviate the inactivation of $\mathrm{Bcl} 2$ and caspases. $\beta$ HB also inhibits HDACs, which regulate FOXOs for metabolic stress and BDNF for neuron growth. BDNF, brain-derived neurotrophic factor; HDACs, histone deacetylases; PP2A, protein phospholipase 2A. Color images are available online. 
$\beta$-HB-enriched ketogenic diets, which account for $80 \%$ to $90 \%$ of high fat, are low in protein and low in carbohydrate diets that meet growth needs (399). Studies by ourselves and others have shown that unlike the HFD, which has certain negative effects (such as the risk of cardiovascular disease and cognitive impairment) despite the fact that the induced body weight gain improved bone health in healthy women and female $\mathrm{AD}$ mice $(183,309)$, ketogenic diets show potential for $\mathrm{AD}$ intervention based on the neuroprotective effect of ketone bodies. A ketogenic diet has been used for 80 years to cure epilepsy. The therapeutic effect might be related to mitochondrial bioenergy improvement, because the ketogenic diet enhances the genes and activities of enzymes in mitochondrial respiration, the TCA cycle, and mitochondrial biogenesis (267).

Microglia metabolize acetoacetate and $\beta$-HB produced by the ketogenic diet, and they respond to these ketones as metabolic signals (122). Studies have shown that the ketogenic diet has protective effects on neurodegenerative diseases, such as $\mathrm{AD}(461,475)$. Low-carbohydrate diets were given to older adults with MCI, and the results showed that patient memory was improved and that the improvement was positively correlated with ketone body levels (72).

The increased risk of AD is associated with dietary habits of high levels of saturated fat and simple carbohydrates. A recent study modified a ketogenic diet with a Mediterranean diet, which makes up for the lack of higher carbohydrates in the ketogenic diet and adds intake of vegetables and fruits, while increasing fats and proteins derived from healthy sources such as olive oil and fish, and the resulting diet showed the potential to ameliorate AD (288).

Fasting leads to the conversion of glucose from glycogen reserve to ketone from fatty acids. It has been reported that in an $\mathrm{AD}$ mouse model, intermittent fasting (IF) protects against GABAergic synaptic adaptations and cognitive deficits, which is mediated by the mitochondrial protein deacetylase SIRT3 (240). SIRT3 has been reported to regulate ketogenesis through activation of mitochondrial HMGCS2 in the fasting state (361). IF also protected against disturbances in energy, glucose, lipid, and bone metabolism in ovariectomized AD rats (362). However, IF could not ameliorate amyloid peptide deposition in $3 \times \mathrm{AD}$ mice (147). Further clinical trials of IF in the early stages of human $\mathrm{AD}$ are necessary.

7. Hydrogen. Hydrogen $\left(\mathrm{H}_{2}\right)$ reacts with specific biomolecules at body temperature. Its high diffusivity allows it not only to rapidly penetrate the BBB but also to enter the cytoplasm, mitochondria, nucleus, ER, and other subcellular structures through the cell membrane structure to interact with target molecules.

The mild reducibility of $\mathrm{H}_{2}$ contributes to its selective reduction of hydroxyl radicals $(\cdot \mathrm{OH})$ and its low disturbance of redox balance in vivo $(194,299)$. Clinical trials have also observed no obvious side effects or $\mathrm{H}_{2}$ toxicity. $\mathrm{H}_{2}$-rich water, $\mathrm{H}_{2}$-rich saline, and $\mathrm{H}_{2}$ inhalation have antioxidant activities similar to those of molecular $\mathrm{H}_{2}$ (262). Our previous study applied coral calcium hydride $(\mathrm{CCH})$, a new solid molecular $\mathrm{H}_{2}$ carrier made of coral calcium, to HFD-induced fatty liver rats and found that $\mathrm{CCH}$ could prevent glucose metabolic disorder via PI3K/Akt signaling and lipid metabolic disorder through ACC/FAS signaling and activate phase 2 enzymes and improve mitochondrial function (164).
In addition to antioxidant activity, the neuroprotective effects of $\mathrm{H}_{2}$ have been studied in recent years. Highly diffusible $\mathrm{H}_{2}$ gas inhalation protects against ischemiareperfusion and stroke-induced oxidative stress by targeting intracellular sources of ROS (299). We found that small amounts of $\mathrm{H}_{2}$ inhalation ameliorated survival and neurological outcomes, including consciousness, cranial nerve reflexes, and sensory function, in the asphyxia rat model of cardiac arrest, and the beneficial effect was superior to that of mild hypothermia (414).

$\mathrm{H}_{2}$-enriched saline was found to ameliorate memory impairment in $\mathrm{A} \beta$-induced $\mathrm{AD}$ mice (223) and to exert antiinflammatory and antioxidant effects on the mouse brain (409). In the injured rat brain, $\mathrm{H}_{2}$-rich saline was found to reverse brain health by suppressing the inflammatory response involving the NF- $\kappa \mathrm{B}$ pathway and NLRP3 inflammasome (352). This finding suggests that molecular $\mathrm{H}_{2}$ may play a beneficial role in protecting against the pathology of $\mathrm{AD}$. Based on a previous $\mathrm{H}_{2}$ study, we speculated that $\mathrm{H}_{2}$ may ameliorate $\mathrm{AD}$ via the mitochondrial metabolic pathway and the anti-inflammatory response. Our recent study found that $\mathrm{H}_{2}$-enriched water significantly improved cognitive dysfunction in female mice via the estrogen-ER $\beta$-BDNF signaling pathway and that $\mathrm{H}_{2}$-enriched water also exerted antioxidant and anti-inflammatory effects in APP/PS1 mice (163). As a newly identified small molecule playing a key role, the potential mechanism by which molecular hydrogen regulates the AD-related pathway needs to be elucidated (Fig. 11).

8. Other mitochondrial nutrients for $A D$. The nutrients administered to $\mathrm{AD}$ patients are typically antioxidants. The antioxidant vitamins $\mathrm{A}, \mathrm{C}$, and $\mathrm{E}$ reduced the risk of $\mathrm{AD}$ (455). The publications regarding the effects of vitamins on

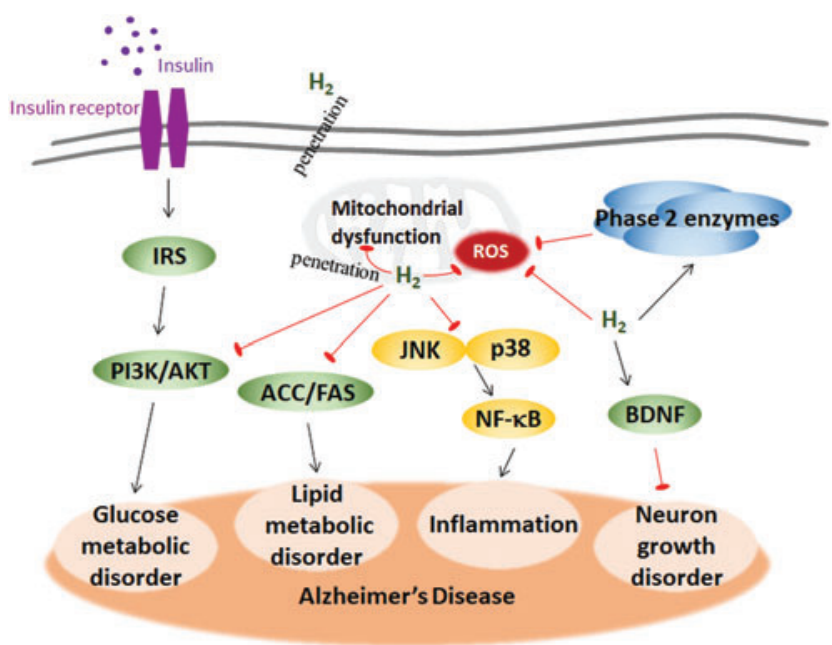

FIG. 11. Functions of hydrogen in AD. The high diffusivity of $\mathrm{H}_{2}$ allows it to not only rapidly penetrate the $\mathrm{BBB}$ but also enter the cytoplasm, mitochondria, and other subcellular membrane compartments to interact with target molecules. Besides the ROS scavenging, $\mathrm{H}_{2}$ prevents glucose metabolic disorder via PI3K/AKT signaling, prevents lipid metabolic disorder through ACC/FAS signaling, prevents inflammation through JNK/p38-NF- $\kappa$ B signaling, activates phase 2 enzymes, and activates BDNF signaling to improve neuron growth. $\mathrm{H}_{2}$, hydrogen. Color images are available online. 
AD are mostly association studies in AD patients, whereas indepth studies on the mechanism are rare. One possible reason underlying the effect of vitamins on AD is that they are involved in multiple metabolic processes. The excessive intake of vitamin $\mathrm{A}$ in the body causes poisoning, and $\beta$-carotene is a safe source of vitamin A because it can be converted to vitamin A after entering the body when needed. Studies have shown that $\beta$-carotene significantly improves behavioral abilities, learning, memory, and cognitive function in mice and rats through its antioxidant effect by scavenging free radicals $(3,457)$.

The first-line antioxidant vitamin $\mathrm{C}$ functions in reduction and hydroxylation reactions. Vitamin $\mathrm{C}$ deficiency in the brain accelerates amyloid pathogenesis and cognitive behavior in APP/PS1 mice, likely through oxidative stress pathways (81). Vitamin E also has the effect of scavenging free radicals, inhibiting lipid peroxidation, and maintaining the integrity and stability of the membrane structure. Vitamin $\mathrm{E}$ is especially abundant in the mitochondrial inner membrane and reacts rapidly with oxidants. Studies have shown that vitamin E significantly improves memory disorders and increased SOD activity (234). However, the clinical roles of vitamin $\mathrm{A}, \mathrm{C}$, and vitamin $\mathrm{E}$ in the prevention and treatment of $\mathrm{AD}$ are still under debate.

The standardized ginkgo biloba extract EGb761 was found to reverse the decrease in ATP production, thereby inhibiting mitochondrial dysfunction and oxidative damage in PC12 cells overexpressing the $\mathrm{A} \beta$ precursor protein $(1,92)$. Polyphenols could regulate the expression of antiapoptotic proteins in the Bcl-2 family to some extent and reduce apoptosis. Epigallocatechin-3-gallate (EGCG) has a certain protective effect on AD cell models and patients. EGCG intervention effectively reduces the decrease in mitochondrial membrane potential and cell apoptosis induced by $\mathrm{A} \beta(12,464)$.

9. Combination of mitochondrial nutrients. The combination of mitochondrial nutrients ameliorates age-related cognitive dysfunction. An optimal combination of mitochondrial nutrients might be more effective than the individual nutrients in the prevention and treatment of $\mathrm{AD}$. Combinations of multiple mitochondrial nutrients have been tested in different mitochondrial dysfunction-related disorders. For example, we initially reported that the combination of LA and ALCAR remarkably improved the cognition of aged rats. The combination of nutrients synergistically ameliorates mitochondrial enzyme activity and cerebral oxidative stress $(235,236)$. The efficiency of ApoE4 to transport cholesterol in neurons decreases under stress conditions. LA has anticholesterol activity, whereas ALCAR reduces cholesterol levels in the brain and blood; therefore, the combination of LA and ALCAR significantly improves cognitive behavior in transgenic ApoE4 mice (207, 356). The combination of ALCAR and donepezil or rivastigmine, cholinesterase inhibitors used for clinical AD treatment, could exert potent effects on the inhibitors of patients with mild AD who are not sensitive to cholinesterase inhibitors, indicating that a combination of anti-AD drugs and mitochondrial nutrients could be an effective strategy for AD treatment (28).

We found that the combination of LA, ALCAR, HT, and $\mathrm{CoQ}_{10}$ ameliorated skeletal muscle atrophy through the activation of mitochondrial biogenesis and reduction of oxidative stress (237). Previous studies have shown that the LA,
ALCAR, nicotinamide, and biotin combination ameliorated the abnormal activities of mitochondrial complexes, mitochondrial biogenesis, oxidative stress, antioxidant defenses, and apoptosis, leading to the reversal of spleen, thymus, liver, and muscle dysfunctions in GK diabetic rats without causing weight gain $(149,150,353)$. A combination of LA and ALCAR not only effectively protected cells against rotenoneinduced mitochondrial dysfunction, oxidative damage, and the accumulation of $\alpha$-synuclein and ubiquitin but also prevented mitochondrial oxidative damage in a cell culture model of Parkinson's disease in primary cells from the brains of aged rats.

ApoE-deficient mice fed a regimen that included vitamin E, ginkgo biloba, pycnogenol, and ascorbyl palmitate had significantly prolonged lifespans and reduced inclusion body histopathology in the hippocampus (398). Meta-analysis indicated that a dietary intake of $\beta$-carotene, vitamin $\mathrm{C}$, and vitamin $E$ lowered the risk of $\mathrm{AD}$ (222). Although vitamins $\mathrm{A}$, $\mathrm{C}$, and $\mathrm{E}$ are still controversial in the prevention and treatment of $\mathrm{AD}$, clinical studies have shown that supplementation with vitamin $\mathrm{E}$ and vitamin $\mathrm{C}$ helps prevent vascular dementia, improves cognitive ability, and reduces the risk of $\mathrm{AD}$; supplementation with vitamin $\mathrm{E}$, vitamin $\mathrm{C}$, and selenium contributes to $\mathrm{AD}$ prevention due to the antioxidative functions of these molecules (97). The findings from our studies and others support the hypothesis that the combination of mitochondrial nutrients or the combination of mitochondrial nutrients with other drugs is an effective strategy to prevent or treat neurodegenerative diseases, such as $\operatorname{AD}(235,236,241,458)$.

\section{B. Exercise}

Similar to mitochondria-targeted nutritional intervention for $\mathrm{AD}$, exercise advantageously has fewer side effects and better dependability than drug treatment; the key point is that exercise improves energy metabolism-centering mitochondria. Several studies in patients with $\mathrm{AD}$ and $\mathrm{AD}$ transgenic mice reported that exercise improved cognitive behaviors and ameliorated AD pathology $(54,98,159,232)$. In middleaged healthy adults, exercise promotes cognitive function and reduces the risk of dementia in later life (76). Exercise targeting energy metabolism mimics the function of mitochondrial nutrients and vice versa. Consequently, exercise is considered an effective strategy for the prevention and adjuvant treatment of AD.

1. Exercise improves the metabolic profile in AD. Exercise improves the whole-body metabolic profile and brain health and, to some extent, decreases AD risk (290, 345), and several potential mechanisms have been found. BDNF regulates nerve cell survival, differentiation, and plasticity, improving cognitive function, learning, and memory, which are beneficial for $\mathrm{AD}$. The release of BDNF enhances not only the function of nerve cells but also the cleavage of APP by $\alpha$-secretase and inhibits $\beta$-secretase, reducing the production of toxic $\mathrm{A} \beta$ peptides $(146,296)$. Exercise was demonstrated to increase BDNF expression by activating the ketone body $\beta$ - $\mathrm{HB}$ in the liver, inducing the activities of Bdnf promoters by HDAC2 and HDAC3 and leading to the neurotransmitter release (367). Blocking BDNF signaling by its receptor TrkB diminishes the positive effect of exercise, which confirms the role of BDNF signaling in the neuroprotective function of exercise $(296,367)$. 
In addition to evidence showing that exercise slows ADrelated pathological features in the brain, exercise could further prevent and alleviate cognitive function in $\mathrm{AD}$ by regulating blood pressure. Aerobic exercise induces endothelium-dependent vasodilatation by upregulating NO production and cardiovascular fitness, thereby promoting neuronal structure and function, mitigating cognitive impairment, and reducing brain atrophy in $\mathrm{AD}(37,161)$. Exercise-derived circulating exosomes elevate miR-342-5p to afford cardioprotection against ischemia/reperfusion injury and regulate neural stem cell proliferation and astrocyte commitment $(115,165)$. Elevated miR-342-5p expression also downregulates the ankyrin G-induced axon initial segment in transgenic $\mathrm{AD}$ mice (376). Exercise reverses $\mathrm{AD}$ related hippocampal decline through the induction of growth factors such as BDNF, IGF-I, and vascular endothelial growth factor (VEGF), in which IGF and VEGF from the periphery could pass through the BBB to promote neurogenesis and angiogenesis $(65,174)$. Exercise also increases glucose uptake in the hippocampus and enhances mitochondrial OXPHOS to produce ATP (400). PI3K/Akt signaling is hyperactivated in $\mathrm{AD}$ and contributes to Tau pathology. Treadmill exercise in AD mice increases PI3K/Akt phosphorylation, reduces downstream GSK-3 $\beta$ activity, reduces Tau protein hyperphosphorylation, and increases neuronal survival by elevating the $\mathrm{Bcl}-2 / \mathrm{Bax}$ ratio and inhibiting the activities of caspase 3 and $\mathrm{COX}-2$, which are induced by $\mathrm{A} \beta$ $(192,203)$.

2. Exercise improves mitochondrial remodeling in $A D$. Exercise improves mitochondrial remodeling and induces mitochondrial biogenesis through the hippocampal PGC-1 $\alpha /$ FNDC5/BDNF pathway to stimulate Bdnf gene expression (431). Exercise enhances neurological health through SIRT1/ PGC- $1 \alpha$, increases citrate synthase, the rate-limiting enzyme in the TCA cycle, and induces mtDNA production, leading to mitochondrial biogenesis (373). Exercise also improves electron transport chain function by increasing complex I activity and improves mitochondrial dynamics by upregulating Drp1 levels (142). Swim training regulates mitochondrial quality control by inhibiting LC-3b turnover-mediated autophagy and PINK1/Parkin-mediated mitophagy in aged mice (465). Regular maternal exercise during pregnancy has long-lasting effects on metabolic programming in the brains of offspring and increases BDNF levels, specifically in mitochondrial function as indicated by increased mitochondrial membrane potential and mass, activates $\alpha$-ketoglutarate dehydrogenase and complex IV, and enhances Mfn1 and Drp1 levels, which then protect against $\mathrm{A} \beta$-induced neurotoxicity and the cognitive impairment of the offspring in their lifetime (202).

3. Exercise decreases inflammation in AD. Exercise also systemically decreases inflammatory markers. The proinflammatory mediators, $\mathrm{COX}-2$ and iNOS and cytokines TNF- $\alpha$, IL- $1 \beta$, and IL- 6 are attenuated by exercise via the MAPK (p-p38 and p-ERK1/2)-dependent NF- $\kappa$ B pathway in transgenic AD mice with Tau abnormalities (219). The p38MAPK pathway, TNF- $\alpha$, and IL- $1 \alpha$ are activated by exercise in PS1 transgenic mice (191) (Fig. 12).

Although several clues and experimental evidence indicate that exercise reduces the pathological features associated with $\mathrm{AD}$, the current research on $\mathrm{AD}$ in the field of sports science and medicine is still rare and not yet in-depth. Because exercise and mitochondrial nutrients have similar benefits for $\mathrm{AD}$, the study of mitochondrial nutrients may provide some clue regarding the mechanism. The specific molecular mechanism by which exercise regulates energy metabolism and further prevents, delays, or slows AD may help to fully understand the occurrence and development of AD systemically.

\section{Natural compounds from traditional Chinese medicines}

As AD is a multifactorial systemic disease with metabolic disturbance in central and peripheral tissues involving insulin resistance, inflammation, and oxidative stress, approaches aiming at either single molecular targets, such as $\mathrm{A} \beta$ and the p-Tau protein, might not be effective in treating $\mathrm{AD}$, and numerous clinical trials have provided fruitless evidence of this notion.

Traditional Chinese medicines (TCMs) undergo a trialerror paradigm, and the effective compounds are active in maintaining bioenergy and metabolic homeostasis and are more likely to maintain mitochondrial energic metabolism and function, for example, agents that may partially rescue the entirety of the metabolic disorders may prevent the progression of $\mathrm{AD}$ pathogenesis. Therefore, the alternative strategy for $\mathrm{AD}$ prevention and treatment may lie in TCMs.

Douglas Wallace proposed mitochondria as chi, which can be translated as "vital force or energy," and emphasized that the Asian herbal medications that target the mitochondria could be used to treat age-related and neurodegenerative diseases, such as $\mathrm{AD}$ (406). Therefore, based on the mitochondria-chi theory, we have associated "chi" with the mitochondrial studies in our laboratory. In addition, according to the traditional concept, Yin and Yang represent the basic opposition of everything. In TCM, all physical structures of the human body can be divided into the corresponding parts of Yin/Yang. Normal life activities are the result of the coordination between Yin and Yang. Once existence Yin/Yang is abnormally strong or weak, it will cause the occurrence of disease.

As shown in Figure 13, when mitochondria are damaged, chi are diminished, resulting in a lack of energy supply to the body; therefore, AD continually worsens. Using TCMs targeting mitochondria as well as mitochondria-induced oxidative stress, inflammation, insulin resistance, and apoptosis constitute a new idea for slowing down or treating AD.

According to numerous scientific articles on Chinese medicine and a long history of clinical practice, several TCMs have shown certain protective effects in treating metabolic disorders and cognitive impairments. Some of these studies have recently been verified, greatly facilitating the screening of promising compounds for $\mathrm{AD}$ prevention. A list of TCM nutraceuticals with mitochondria function-related readouts is presented in this section (Table 2). We listed mitochondrial dysfunction, oxidative stress, inflammatory disorder, insulin resistance, and hepatic metabolic dysfunction as representative mitochondrial function-related readouts (Fig. 13).

1. TCMs ameliorate insulin resistance and hepatic metabolic dysfunction. Ginseng is the most commonly mentioned TCM with broad beneficial effects on the CNS and the 


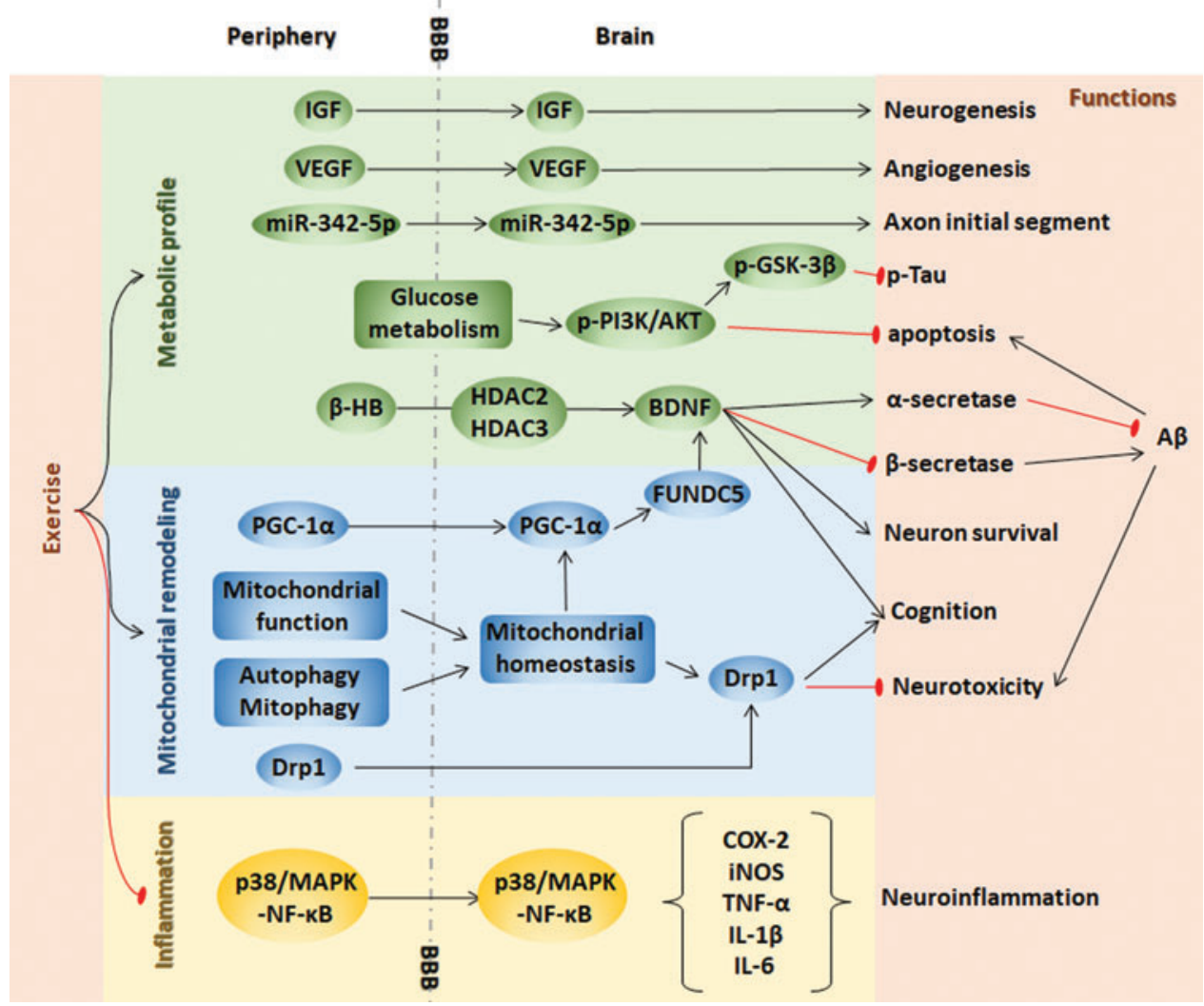

FIG. 12. Functions of exercise in AD. Exercise improves energy metabolism by centering mitochondria to ameliorate AD pathological progression. (i) Exercise improves the metabolic profile in AD. The induced IGF and VEGF in the periphery pass through the BBB to promote neurogenesis and angiogenesis, respectively. The elevated miR-342-5p crosses the BBB to regulate the axon initial segment. The increased glucose uptake hyperactivates PI3K/AKT signaling to contribute to Tau pathology. Activated $\beta$-HB induces BDNF signaling by HDAC2 and HDAC3. BDNF signaling participates in neuroprotective functions, including activating $\alpha$-secretase, inhibiting $\beta$-secretase, promoting neuron survival, and protecting cognition. (ii) Exercise improves mitochondrial remodeling in AD. Exercise induces mitochondrial biogenesis through PGC-1 $\alpha /$ FUNDC5 to stimulate the BDNF pathway. Exercise also improves mitochondrial homeostasis by regulating mitochondrial function, autophagy, and, to some extent, mitophagy. Drp-1 levels enhanced by exercise protect against $\mathrm{A} \beta$-induced neurotoxicity and cognitive function. (iii) Exercise decreases inflammation in AD. Some proinflammatory mediators (COX-2 and iNOS) and cytokines (TNF- $\alpha$, IL-1 $\beta$, and IL-6) are attenuated by exercise through the p38/MAPKdependent NF- $\kappa$ B pathway to systemically decrease inflammation. COX-2, cytochrome c oxidase subunit II; MAPK, mitogen-activated protein kinase. Color images are available online.

cardiovascular, endocrine, and immune systems $(17,199)$. Ginsenoside $\mathrm{Rg} 1$, the major active component in ginseng, is effective in recovering insulin sensitivity and reducing blood glucose levels in diabetic db/db mice and ob/ob mice, and the mechanism was also investigated in insulin-resistant cell models in which ginsenoside $\mathrm{Rg} 1$ activates the AMPK pathway $(218,439,440)$. In addition, ginsenoside $\operatorname{Rg} 1$ also increases insulin-degrading enzymes, decreases $\mathrm{A} \beta$ levels, and improves learning and memory in a rat model injected with $\mathrm{A} \beta_{1-42}$ (320). Curcuma longa $\mathrm{L}$ (turmeric) is another TCM that is mainly used to treat abdominal pains (14). Curcumin, a polyphenolic compound derived from turmeric, ameliorates insulin resistance and glucose homeostasis in diabetic $\mathrm{db} / \mathrm{db}$ mice by activating glycolysis and inhibiting gluconeogenic and lipid metabolic enzymes in the liver (350). Further, curcumin effectively reduces the plaque burden and insoluble $\mathrm{A} \beta$ accumulation in Tg2576/APPsw mice and protects against $\mathrm{A} \beta$-induced mitochondrial dysfunction in neurons by inhibiting GSK-3 $\beta(25,169)$. These results suggest that TCM nutraceuticals that ameliorate insulin resistance, such as ginsenoside $\operatorname{Rg} 1$ and curcumin, have the potential to prevent AD.

As reviewed earlier, compromised hepatic metabolic function is an early event that plays a key role in the pathogenesis of $\mathrm{AD}$, and nutraceuticals/drugs that preserve normal hepatic function should be another type of candidate for treating AD. Salvia miltiorrhiza Bunge in TCM promotes blood circulation to remove blood stasis (301). Cryptotanshinone (CTS), a major constituent extracted from S. miltiorrhiza Bunge, decreases apoptosis and inflammation and ameliorates reduced liver failure (182). In addition, CTS prevents cognitive decline in APP/PS1 mice (271).

2. TCMs ameliorate mitochondrial dysfunction and oxidative stress. The TCM Huperziaserrata has been used for more than 1000 years in China for the treatment of numerous of ailments, including contusions, schizophrenia, swelling, myasthenia gravis, and, most recently, organophosphate 


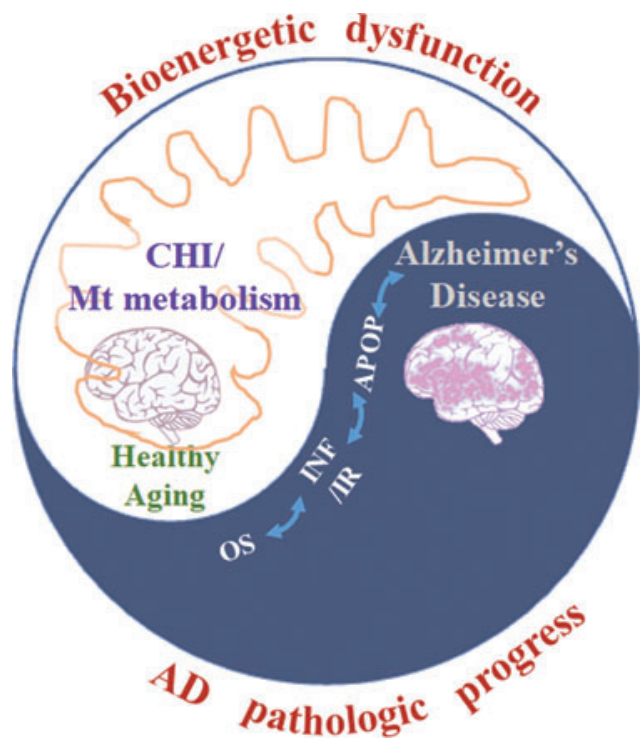

FIG. 13. An alternative strategy for AD prevention and treatment may lie in TCMs. Yin and Yang represent the basic opposition of everything in the Chinese traditional concept; especially in the TCMs, all physical structures of the human body can be divided into the corresponding parts of Yin/Yang. Wellness is based on the coordination between Yin and Yang. Once the existence of Yin/Yang is abnormally strong or weak, it will cause the occurrence of disease. Mitochondrion, "chi" equivalent in Chinese Medicine, is considered as "Yang" in maintaining the energy forces of the living cell, whereas ROS generation, lowered respiration, apoptosis, etc. are regarded as "Yin." Once mitochondria are impaired by the internal and external stress, the "chi" is losing along; meanwhile, the peripheral/ central progressive oxidative stress, inflammation, insulin resistance, and eventual neuronal apoptosis progressively occur, leading to the imbalance of Yin and Yang, which facilitates $\mathrm{AD}$ pathogenesis and progression. Using TCMs that target mitochondria-related processes, including favoring mitochondrial function, such as stimulating biogenesis and degradation, respiration, or suppressing oxidative stress, inflammation, and apoptosis, may be an alternative way to slow down or treat AD progression. APOP, apoptosis; INF, inflammation; MD, metabolic disease; MT, mitochondria; OS, oxidative stress. Color images are available online.

poisoning (422). Huperzine-A, a component derived from Huperziaserrata, protects mitochondria from $\mathrm{A} \beta$-induced damage, at least in part by preserving membrane integrity, inhibiting oxidative stress and improving energy metabolism $(117,118)$. Huperzine-A inhibits the neurotoxicity of $\mathrm{A} \beta$ and improves memory in patients with AD $(437,459,460)$.

Gynostemma pentaphyllum has various pharmacological effects, such as detoxification and heart palpitation clearance abilities (324). Gypenoside, a main bioactive ingredient in $G$. pentaphyllum, was reported to improve the cognitive function of an $\mathrm{A} \beta$-induced AD rat model, probably by scavenging free radicals, inhibiting oxidative damage, and maintaining normal mitochondrial ultrastructure (446, 470). The TCM Moutan Cortex is typically used to treat diseases, such as atherosclerosis, infection, and inflammation, among many others (432). Paeonol, isolated from the bark of Moutan Cortex, possesses anti-inflammatory and antioxidant prop- erties and has been reported to increase COX-1 expression and improve the cognitive function of $\mathrm{A} \beta$-induced $\mathrm{AD}$ rats (471).

3. TCMs ameliorate inflammation. Central and peripheral inflammatory activation is featured during the progression of AD. Some TCM nutraceuticals have demonstrated robust anti-inflammatory activity. Forsythia suspensa (Thunb.) Vahl is a climbing plant, the fruit of which is a famous TCM commonly used in clinical treatments for gonorrhea, erysipelas, inflammation, pyrexia, and ulcer (435). Forsythiaside, isolated from $F$. suspensa, has been reported to improve cognition and memory in accelerated aging mice and SAMP8 mice, by suppressing oxidative stress and decreasing inflammatory cytokines (411). The CTS mentioned earlier also has an antiinflammatory effect $(293,386)$. These studies imply that the constituents with anti-inflammatory or antioxidant properties may partially relieve symptoms of AD by improving oxidative stress or decreasing inflammation.

4. A variety of TCMs with mitochondria-related functional readouts may reveal a new therapeutic strategy in $A D$ treatment. Given that complex metabolic disorders interact and progress from early to late stages of $\mathrm{AD}$, the TCMs that rescue more than one $\mathrm{AD}$-related critical metabolic disorder may be better candidates and be more helpful for AD treatment (Fig. 12).

In addition to the TCMs mentioned earlier, other TCMs, such as Coptis chinensis Franch, berberine, and Sophora flavescens Ait, also show benefits in both metabolic dysfunction and $\mathrm{AD}$ treatment $(71,170)$. Berberine, a major isoquinoline alkaloid in $C$. chinensis Franch, an important TCM herb that has been widely used (444), has multiple pharmacological functions, including anti-inflammatory, antioxidant, antidiabetic, liver-protective, and neuroprotective activities. Berberine has been reported to efficiently reduce cerebral $\mathrm{A} \beta$ levels and glial activation and significantly ameliorate cognitive impairment in TgCRND8 mice (90). Other recent studies have also found that berberine can additionally treat senile dementia by improving antioxidative stress, metabolism, and other multitarget pathways (170).

Matrine, the major active component of S. flavescens, which is typically used to treat dementia and exerts multiple pharmacological functions similar to berberine, has been found to be efficient in improving cognitive deficits in APP/ PS1 mice by inhibiting A $\beta$ aggregation in vivo and blocking the $\mathrm{A} \beta / \mathrm{RAGE}$ axis (71). These two components not only recover the metabolic symptoms associated with AD but also reduce $\mathrm{A} \beta$ levels.

Although most of the studies described earlier were conducted in cellular and animal AD models, a systemic survey of TCMs using a variety of mitochondrial functional readouts may reveal new therapeutic strategies (406) based on clues accumulating from Chinese medicine practices. TCMs and some other herbal medicines are occasionally challenged due to a lack of rigorous scientific studies. It has been proposed that TCMs may act through hormetic dose-response mechanisms (410). Hormesis is a type of dose-response phenomenon that is characterized by low-dose stimulation and high-dose inhibition (40). As the right dose is critical for successful nutrient intervention, further research attention should be paid to TCM doses and more studies on the molecular mechanism are needed. 
Table 2. Potential Nutraceuticals from Traditional Chinese Medicines for Alzheimer's Disease Prevention And Treatment

\begin{tabular}{|c|c|c|c|c|c|}
\hline \multirow[b]{2}{*}{$T C M s$} & \multicolumn{3}{|c|}{ Experiment involving $A D$} & \multirow{2}{*}{$\begin{array}{l}\text { The functional target } \\
\text { mitochondrial } \\
\text { dysfunction or } A D\end{array}$} & \multirow[b]{2}{*}{ References } \\
\hline & Purity & Doses & Administration & & \\
\hline Ginsenoside $\operatorname{Rg} 1$ & $98 \%$ & $10 \mathrm{mg} / \mathrm{kg}$ & $\begin{array}{l}\text { Intraperitoneal } \\
\text { injection }\end{array}$ & $\begin{array}{l}\text { Insulin sensitivity; blood } \\
\text { glucose; IDE; A } \beta \text {; } \\
\text { learning and memory }\end{array}$ & $\begin{array}{l}(218,320 \\
439,440)\end{array}$ \\
\hline Curcumin & - & $100-400 \mathrm{mg} / \mathrm{kg}$ & $\begin{array}{l}\text { Orally } \\
\text { administered } \\
\text { by gavage }\end{array}$ & $\begin{array}{l}\text { IR; glucose homeostasis; } \\
\text { glycolysis; gluconeogenic } \\
\text { and lipid metabolic } \\
\text { enzymes; } A \beta\end{array}$ & $(25,350,415)$ \\
\hline Cryptotanshinone & $\geq 97 \%$ & $1-10 \mathrm{mg} / \mathrm{kg}$ & $\begin{array}{l}\text { Intraperitoneal } \\
\text { injection }\end{array}$ & Apoptosis; inflammation & $(182,255,271)$ \\
\hline Huperzine-A & $>99 \%$ & $0.2 \mathrm{mg} / \mathrm{kg}$ & $\begin{array}{l}\text { Intraperitoneal } \\
\text { injection }\end{array}$ & $\begin{array}{l}\text { Mitochondrial membrane } \\
\text { integrity; oxidative stress; } \\
\text { energy metabolism; } \\
\text { neurotoxicity of } \mathrm{A} \beta\end{array}$ & $\begin{array}{c}(117,118 \\
437,459,460)\end{array}$ \\
\hline $\begin{array}{l}\text { Gynostemma } \\
\text { pentaphyllum } \\
\text { makino }\end{array}$ & $>98 \%$ & $50-250 \mathrm{mg} / \mathrm{kg}$ & $\begin{array}{l}\text { Orally } \\
\text { administered } \\
\text { by gavage }\end{array}$ & $\begin{array}{l}\text { Cognitive function; free } \\
\text { radicals; mitochondrial } \\
\text { ultrastructure }\end{array}$ & $(445,446,470)$ \\
\hline Paeonol & $>98 \%$ & $5 \mathrm{mg} / \mathrm{kg}$ & $\begin{array}{l}\text { Intraperitoneal } \\
\text { injection }\end{array}$ & $\begin{array}{l}\text { Inflammation; cognitive } \\
\text { function }\end{array}$ & $(471)$ \\
\hline Forsythiaside & - & $60-240 \mathrm{mg} / \mathrm{kg}$ & Orally & $\begin{array}{l}\text { Cognition and memory } \\
\text { ability; oxidative stress; } \\
\text { inflammatory cytokines }\end{array}$ & (411) \\
\hline Berberine & $\begin{array}{c}\text { Berberine } \\
\text { hydrochloride } \\
98 \%\end{array}$ & $25-100 \mathrm{mg} / \mathrm{kg}$ & $\begin{array}{l}\text { Orally } \\
\text { administered } \\
\text { by gavage }\end{array}$ & $\begin{array}{l}\text { Inflammation; neuroprotec- } \\
\text { tive; } \mathrm{A} \beta \text {; glial activation; } \\
\text { cognitive impairment; } \\
\text { neurotransmitter; } \\
\text { oxidative stress }\end{array}$ & $(90,91,170)$ \\
\hline Matrine & - & $50-100 \mathrm{mg} / \mathrm{kg}$ & $\begin{array}{l}\text { Intraperitoneal } \\
\text { injection }\end{array}$ & $\mathrm{A} \beta ; \mathrm{A} \beta / \mathrm{RAGE}$ axis & (71) \\
\hline
\end{tabular}

A $\beta$, beta amyloid; AD, Alzheimer's disease; IDE, insulin degrading enzyme; IR, insulin receptor; RAGE, receptor for advanced glycation end products; TCM, traditional Chinese medicine.

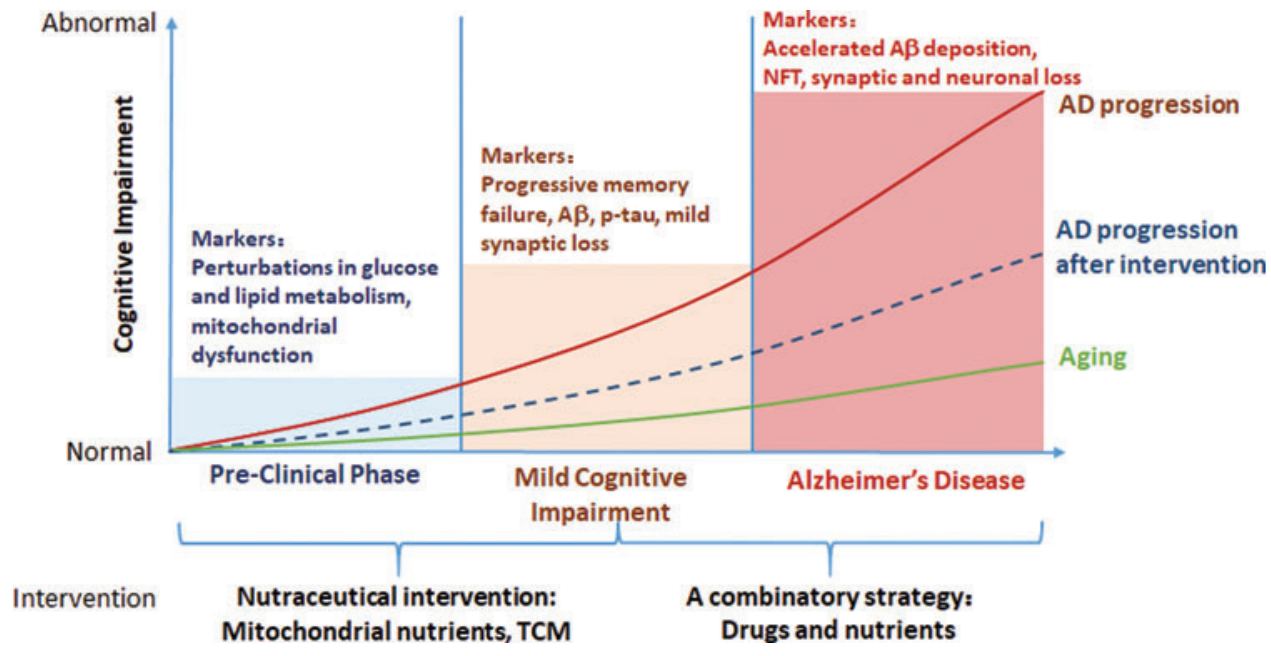

FIG. 14. Early intervention might be more effective at slowing AD progression. Perturbations in glucose metabolism, lipid metabolism, and mitochondrial dysfunction in $\mathrm{AD}$ occur even earlier than the preclinical phase of $\mathrm{AD}$. Mitochondria play an important role in manipulating glucose and lipid metabolism during the occurrence and development of AD. Therefore, mitochondrial nutrients will be a possible means to delay the progression of $\mathrm{AD}$ at this stage. However, $\mathrm{AD}$ cannot be cured by the current existing drugs. After the diagnosis of AD by its pathological features, such as A $\beta$ deposition, NFTs, synaptic and neuronal loss, mitochondrial nutrients can also be combined with current drugs to improve the efficiency, finally delaying $\mathrm{AD}$ progression. Color images are available online. 
FIG. 15. Treating AD with multiple targets focusing on mitochondria. The idea implies a promising strategy to prevent, delay, or ameliorate $\mathrm{AD}$ progression by the use of multiple targets centered on the balanced energy metabolism of mitochondria, including decreasing oxidative stress, insulin resistance, and inflammation, and increasing phase II enzymes of antioxidant defenses, mitochondrial homeostasis, and mitochondrial enzyme activities in the brain and periphery. Ultimately, healthy mitochondria help to reduce the risk of $\mathrm{AD}$, delay the onset of $\mathrm{AD}$, and ameliorate the progression of $\mathrm{AD}$. Color images are available online.

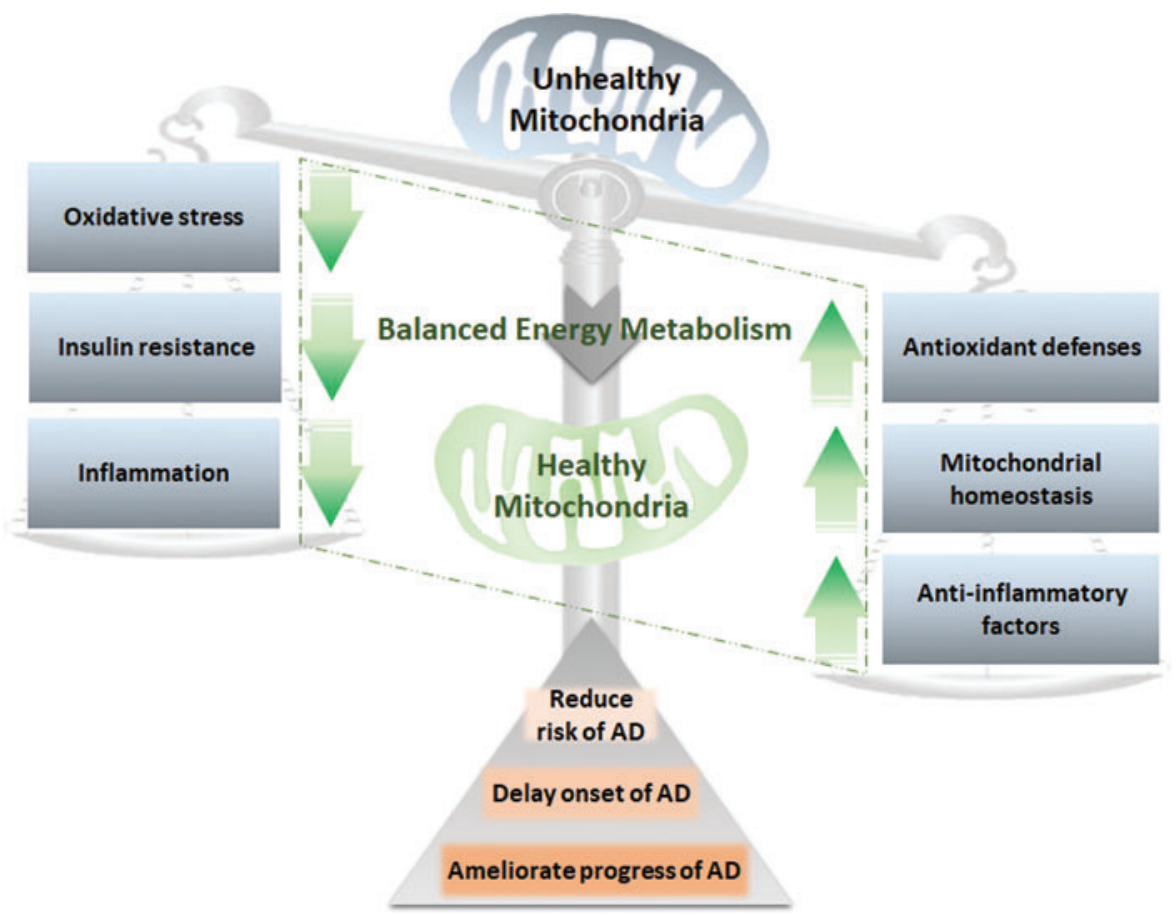

\section{Concluding Remarks}

$\mathrm{AD}$ and metabolic syndrome are persistent challenges to morbidity, mortality, lifestyles, and human life expectancy. $\mathrm{AD}$ is almost incurable at this stage, and there are almost no effective interventions or treatments. The long-term use of the existing drugs is often accompanied by serious adverse side effects. Currently, treatment is aimed at improving cognitive function, working ability, and quality of life. However, the current treatment can hardly delay or reverse the course of this disease, let alone cure it. AD is a complex disease involving many signaling molecules, and overlapping genetic and environmental factors are involved in the development of diseases. Various factors have made the treatment of $\mathrm{AD}$ a challenging and urgent research hotspot.

The pathogenesis of $\mathrm{AD}$ is still unclear. The association between $\mathrm{AD}$ and metabolic syndrome from the evidence of clinical, epidemiological genetic, and molecular discoveries has inspired scientists to investigate the underlying mechanisms. Increasing evidence implies that central and peripheral mitochondrial metabolic deficiency occurs even earlier than the preclinical phase of $\mathrm{AD}$ (Fig. 14). The early diagnosis of $\mathrm{AD}$, indicated by biomarkers, and the early intervention may provide a new strategy for $\mathrm{AD}$ treatment. As an important organelle for regulating the "life" and "death" of cells, mitochondria play an important role in the occurrence and development of $\mathrm{AD}$. Therefore, targeting mitochondria will become a new strategy and an important means for the treatment of AD. Mitochondria are susceptible to the earliest signs of AD pathogenesis; remodeled mitochondrial metabolites represent a potential group of novel peripheral diagnostic markers of $\mathrm{AD}$ and are more readily detected than CNS-oriented markers in bodily fluids.

Mitochondrial nutrients, as well as some compounds from Chinese traditional medicine, ameliorate some metabolic disorders, including mitochondrial metabolic dysfunction, insulin resistance, and inflammatory provocation in $\mathrm{AD}$.
Moreover, the crosstalk between the brain and periphery links metabolic regulation and brain function, which implies a new aspect for identifying therapeutic targets for AD. Therefore, treating AD by targeting mitochondria provides a promising approach to preventing and delaying AD progression (Fig. 15). Mitochondrial-targeted therapy is still at the exploratory stage. Although many studies have shown that this treatment has a certain therapeutic effect, significant clinical improvement has not yet been confirmed. Thus, both clinical experimental and basic research on the pathogenesis of this disease is warranted.

Treatments aimed at a single target or single pathology of $\mathrm{AD}$ are less efficient in $\mathrm{AD}$ medicine development. In contrast, the combined therapy of mitochondrial nutrients or TCMs and exercise synergistically ameliorates mitochondrial-induced oxidative stress, metabolic dysfunction, and inflammation in both the CNS and the periphery and could be more successful than monotherapy.

To date, numerous publications have shown that dietary or lifestyle interventions are associated with the risk of $\mathrm{AD}$ or reduce the progression of $\mathrm{AD}$; however, scientific evidence is limited, and further studies and clinical trials are needed. The mechanism of $\mathrm{AD}$ is still the most important issue to investigate and remains highly prioritized, and the exact treatments needed to cure $\mathrm{AD}$ cannot be discovered until the mechanism has been elucidated.

\section{Acknowledgment}

The authors thank Dr. Byron C. Jones, University of Tennessee for the critical comments and editing.

\section{Funding Information}

This work was supported by the National Basic Research Program of China [973 program nos. 2015CB856302 and 
2015CB553602], the National Natural Science Foundation of China [Nos. 81741110, 31770917, 31570777, 31870848, and 81802787], the China Postdoctoral Science Foundation [no. 2018M643673], and the opening foundation of the State Key Laboratory of Space Medicine Fundamentals and Application, Chinese Astronaut Research and Training Center [no. SMFA15K01].

\section{References}

1. Abdel-Kader R, Hauptmann S, Keil U, Scherping I, Leuner K, Eckert A, and Müller WE. Stabilization of mitochondrial function by Ginkgo biloba extract (EGb 761). Pharmacol Res 56: 493-502, 2007.

2. Accardi G, Caruso C, Colonna-Romano G, Camarda C, Monastero R, and Candore G. Can Alzheimer disease be a form of type 3 diabetes? Rejuvenation Res 15: 217-221, 2012.

3. Ademowo OS, Dias HKI, Milic I, Devitt A, Moran R, Mulcahy R, Howard AN, Nolan JM, and Griffiths HR. Phospholipid oxidation and carotenoid supplementation in Alzheimer's disease patients. Free Radic Biol Med 108: 77-85, 2017.

4. Ahmad F, Singh K, Das D, Gowaikar R, Shaw E, Ramachandran A, Rupanagudi KV, Kommaddi RP, Bennett DA, and Ravindranath V. Reactive oxygen species-mediated loss of synaptic Akt1 signaling leads to deficient activity-dependent protein translation early in Alzheimer's disease. Antioxid Redox Signal 27: 12691280, 2017.

5. Akbar M, Calderon F, Wen Z, and Kim HY. Docosahexaenoic acid: a positive modulator of Akt signaling in neuronal survival. Proc Natl Acad Sci U S A 102: 10858-10863, 2005.

6. Alavian KN, Beutner G, Lazrove E, Sacchetti S, Park HA, Licznerski P, Li H, Nabili P, Hockensmith K, Graham M, Porter GA, Jr., and Jonas EA. An uncoupling channel within the c-subunit ring of the F1FO ATP synthase is the mitochondrial permeability transition pore. Proc Natl Acad Sci U S A 111: 10580-10585, 2014.

7. Alzheimer A, Stelzmann RA, Schnitzlein HN, and Murtagh FR. An English translation of Alzheimer's 1907 paper, "Uber eine eigenartige Erkankung der Hirnrinde". Clin Anat 8: 429-431, 1995.

8. Alzheimer's-Association. 2018 Alzheimer's disease facts and figures. Alzheimers Dement 14: 367-429, 2018.

9. Amato S, Liu X, Zheng B, Cantley L, Rakic P, and Man $\mathrm{H}-\mathrm{Y}$. AMP-activated protein kinase regulates neuronal polarization by interfering with PI 3-kinase localization. Science 332: 247-251, 2011.

10. Anandatheerthavarada HK, Biswas G, Robin MA, and Avadhani NG. Mitochondrial targeting and a novel transmembrane arrest of Alzheimer's amyloid precursor protein impairs mitochondrial function in neuronal cells. J Cell Biol 161: 41-54, 2003.

11. Ancolio K, Dumanchin C, Barelli H, Warter J, Brice A, Campion D, Frebourg T, and Checler F. Unusual phenotypic alteration of $\beta$ amyloid precursor protein ( $\beta$ APP) maturation by a new Val-715 $\rightarrow$ Met $\beta$ APP-770 mutation responsible for probable early-onset Alzheimer's disease. Proc Natl Acad Sci U S A 96: 4119-4124, 1999.

12. André S, Felix H, Bettina WL, Anne Christin MG, Jürgen D, Christoph B, and Stefan B. Green tea extract enhances parieto-frontal connectivity during working memory processing. Psychopharmacology 231: 3879-3888, 2014.
13. Arai $M$, Nobusawa $S$, Ikota $H$, Takemura $S$, and Nakazato Y. Frequent IDH1/2 mutations in intracranial chondrosarcoma: a possible diagnostic clue for its differentiation from chordoma. Brain Tumor Pathol 29: 201-206, 2012.

14. Araujo $\mathrm{C}$ and Leon L. Biological activities of Curcuma longa L. Mem Inst Oswaldo Cruz 96: 723-728, 2001.

15. Archer SL. Mitochondrial dynamics - mitochondrial fission and fusion in human diseases. $N$ Engl J Med 369: 2236-2251, 2013.

16. Arunsundar M, Shanmugarajan TS, and Ravichandran V. 3, 4-Dihydroxyphenylethanol attenuates spatio-cognitive deficits in an Alzheimer's disease mouse model: modulation of the molecular signals in neuronal survivalapoptotic programs. Neurotox Res 27: 143-155, 2015.

17. Attele AS, Wu JA, and Yuan C-S. Ginseng pharmacology: multiple constituents and multiple actions. Biochem Pharmacol 58: 1685-1693, 1999.

18. Augustinack JC, Schneider A, Mandelkow EM, and Hyman BT. Specific tau phosphorylation sites correlate with severity of neuronal cytopathology in Alzheimer's disease. Acta Neuropathol 103: 26-35, 2002.

19. Baek SH, Park SJ, Jeong JI, Kim SH, Han J, Kyung JW, Baik SH, Choi Y, Choi BY, Park JS, Bahn G, Shin JH, Jo DS, Lee JY, Jang CG, Arumugam TV, Kim J, Han JW, Koh JY, Cho DH, and Jo DG. Inhibition of Drp1 ameliorates synaptic depression, Abeta deposition, and cognitive impairment in an Alzheimer's disease model. J Neurosci 37: 5099-5110, 2017.

20. Bagyinszky E, Kang MJ, Van Giau V, Shim K, Pyun J-M, Suh J, An SSA, and Kim S. Novel amyloid precursor protein mutation, Val669Leu ("Seoul APP"), in a Korean patient with early-onset Alzheimer's disease. Neurobiol Aging 84: 236.e1-236.e7, 2019.

21. Balbín M, Abrahamson M, Gustafson L, Nilsson K, Brun $\mathrm{A}$, and Grubb A. A novel mutation in the $\beta$-protein coding region of the amyloid $\beta$-protein precursor (APP) gene. Hum Genet 89: 580-582, 1992.

22. Baloyannis SJ. Mitochondrial alterations in Alzheimer's disease. J Alzheimers Dis 9: 119-126, 2006.

23. Bartlett $\mathrm{K}$ and Eaton S. Mitochondrial $\beta$-oxidation. Eur J Biochem 271: 462-469, 2004.

24. Bateman RJ, Xiong C, Benzinger TL, Fagan AM, Goate A, Fox NC, Marcus DS, Cairns NJ, Xie X, and Blazey TM. Clinical and biomarker changes in dominantly inherited Alzheimer's disease. N Engl J Med 2012: 795804, 2012.

25. Begum AN, Jones MR, Lim GP, Morihara T, Kim P, Heath DD, Rock CL, Pruitt MA, Yang F, and Hudspeth B. Curcumin structure-function, bioavailability, and efficacy in models of neuroinflammation and Alzheimer's disease. J Pharmacol Exp Ther 326: 196-208, 2008.

26. Behl C. Alzheimer's disease and oxidative stress: implications for novel therapeutic approaches. Prog Neurobiol 57: 301-323, 1999.

27. Bejanin A, Schonhaut DR, La Joie R, Kramer JH, Baker SL, Sosa N, Ayakta N, Cantwell A, Janabi M, Lauriola M, O’Neil JP, Gorno-Tempini ML, Miller ZA, Rosen HJ, Miller BL, Jagust WJ, and Rabinovici GD. Tau pathology and neurodegeneration contribute to cognitive impairment in Alzheimer's disease. Brain 140: 3286-3300, 2017.

28. Bianchetti A, Rozzini R, and Trabucchi M. Effects of acetyl-L-carnitine in Alzheimer's disease patients unre- 
sponsive to acetylcholinesterase inhibitors. Curr Med Res Opin 19: 350-353, 2003.

29. Binder LI, Frankfurter A, and Rebhun LI. The distribution of tau in the mammalian central nervous system. J Cell Biol 101: 1371-1378, 1985.

30. Blanch M, Mosquera JL, Ansoleaga B, Ferrer I, and Barrachina M. Altered mitochondrial DNA methylation pattern in Alzheimer disease-related pathology and in Parkinson disease. Am J Pathol 186: 385-397, 2016.

31. Blass JP. The mitochondrial spiral: an adequate cause of dementia in the Alzheimer's syndrome. Ann N Y Acad Sci 924: 170-183, 2000.

32. Blauwendraat C, Wilke C, Jansen IE, Schulte C, SimónSánchez J, Metzger FG, Bender B, Gasser T, Maetzler W, and Rizzu P. Pilot whole-exome sequencing of a German early-onset Alzheimer's disease cohort reveals a substantial frequency of PSEN2 variants. Neurobiol Aging 37: 208.e11-208.e17, 2016.

33. Bomfim TR, Forny-Germano L, Sathler LB, BritoMoreira J, Houzel JC, Decker H, Silverman MA, Kazi H, Melo HM, and McClean PL. An anti-diabetes agent protects the mouse brain from defective insulin signaling caused by Alzheimer's disease-associated A $\beta$ oligomers. J Clin Invest 122: 1339, 2012.

34. Borquez DA, Urrutia PJ, Wilson C, van Zundert B, Nunez MT, and Gonzalez-Billault C. Dissecting the role of redox signaling in neuronal development. J Neurochem 137: 506-517, 2016.

35. Brissot P, Troadec MB, Loreal O, and Brissot E. Pathophysiology and classification of iron overload diseases; update 2018. Transfus Clin Biol 26: 80-88, 2019.

36. Brustovetsky N and Dubinsky JM. Limitations of cyclosporin A inhibition of the permeability transition in CNS mitochondria. J Neurosci 20: 8229-8237, 2000.

37. Burns JM, Cronk BB, Anderson HS, Donnelly JE, Thomas GP, Harsha A, Brooks WM, and Swerdlow RH. Cardiorespiratory fitness and brain atrophy in early Alzheimer disease. Neurology 71: 210-216, 2008.

38. Burns JM, Donnelly JE, Anderson HS, Mayo MS, Spencer-Gardner L, Thomas G, Cronk BB, Haddad Z, Klima D, Hansen D, and Brooks WM. Peripheral insulin and brain structure in early Alzheimer disease. Neurology 69: 1094-1104, 2007.

39. Cadonic C, Sabbir MG, and Albensi BC. Mechanisms of mitochondrial dysfunction in Alzheimer's disease. Mol Neurobiol 53: 6078-6090, 2016.

40. Calabrese V, Cornelius C, Dinkovakostova AT, Calabrese EJ, and Mattson MP. Cellular stress responses, the hormesis paradigm, and vitagenes: novel targets for therapeutic intervention in neurodegenerative disorders. Antioxid Redox Signal 13: 1763-1811, 2010.

41. Cali C, Tauffenberger A, and Magistretti P. The strategic location of glycogen and lactate: from body energy reserve to brain plasticity. Front Cell Neurosci 13: 82, 2019.

42. Calon F, Lim GP, Yang F, Morihara T, Teter B, Ubeda O, Rostaing P, Triller A, Salem N, Jr., Ashe KH, Frautschy SA, and Cole GM. Docosahexaenoic acid protects from dendritic pathology in an Alzheimer's disease mouse model. Neuron 43: 633-645, 2004.

43. Campos-Pena V, Toral-Rios D, Becerril-Perez F, Sanchez-Torres C, Delgado-Namorado Y, Torres-Ossorio E, Franco-Bocanegra D, and Carvajal K. Metabolic syndrome as a risk factor for Alzheimer's disease: is Abeta a crucial factor in both pathologies? Antioxid Redox Signal 26: 542-560, 2017.

44. Canevelli M, Piscopo P, Talarico G, Vanacore N, Blasimme A, Crestini A, Tosto G, Troili F, Lenzi GL, and Confaloni A. Familial Alzheimer's disease sustained by presenilin 2 mutations: systematic review of literature and genotype-phenotype correlation. Neurosci Biobehav Rev 42: 170-179, 2014.

45. Carro E and Torres-Aleman I. Insulin-like growth factor I and Alzheimer's disease: therapeutic prospects? Expert Rev Neurother 4: 79-86, 2004.

46. Carro E and Torres-Aleman I. The role of insulin and insulin-like growth factor I in the molecular and cellular mechanisms underlying the pathology of Alzheimer's disease. Eur J Pharmacol 490: 127-133, 2004.

47. Carter D, Desmarais E, Bellis M, Campion D, ClergetDarpoux F, Brice A, Agid Y, Jaillard-Serradt A, and Mallet J. More missense in amyloid gene. Nat Genet 2: 255, 1992.

48. Chan DC. Mitochondrial fusion and fission in mammals. Annu Rev Cell Dev Biol 22: 79-99, 2006.

49. Chang CR and Blackstone C. Dynamic regulation of mitochondrial fission through modification of the dynaminrelated protein Drp1. Ann NY Acad Sci 1201: 34-39, 2010.

50. Chang S, ran Ma T, Miranda RD, Balestra ME, Mahley RW, and Huang Y. Lipid-and receptor-binding regions of apolipoprotein E4 fragments act in concert to cause mitochondrial dysfunction and neurotoxicity. Proc Natl Acad Sci U S A 102: 18694-18699, 2005.

51. Chartier-Harlin M-C, Crawford F, Houlden H, Warren A, Hughes D, Fidani L, Goate A, Rossor M, Roques P, and Hardy J. Early-onset Alzheimer's disease caused by mutations at codon 717 of the $\beta$-amyloid precursor protein gene. Nature 353: 844, 1991.

52. Chen W-T, Hong C-J, Lin Y-T, Chang W-H, Huang H-T, Liao J-Y, Chang Y-J, Hsieh Y-F, Cheng C-Y, and Liu $\mathrm{H}-\mathrm{C}$. Amyloid-beta $(\mathrm{A} \beta)$ D7H mutation increases oligomeric $\mathrm{A} \beta 42$ and alters properties of $\mathrm{A} \beta$-zinc/copper assemblies. PLoS One 7: e35807, 2012.

53. Chen Y, Shi S, Zhang J, Gao H, Liu H, Wang J, and Lin $\mathrm{H}$. Diagnostic value of AD7C-NTP for patients with mild cognitive impairment due to Alzheimer's disease. Zhonghua Yi Xиe Za Zhi 94: 1613-1617, 2014.

54. Cheng S-T, Chow PK, Song Y-Q, Edwin C, Chan AC, Lee $\mathrm{TM}$, and Lam JH. Mental and physical activities delay cognitive decline in older persons with dementia. Am J Geriatr Psychiatry 22: 63-74, 2014.

55. Chiurchiu V, Orlacchio A, and Maccarrone M. Is modulation of oxidative stress an answer? The state of the art of redox therapeutic actions in neurodegenerative diseases. Oxid Med Cell Longev 2016: 7909380, 2016.

56. Cho DH, Nakamura T, Fang J, Cieplak P, Godzik A, Gu Z, and Lipton SA. S-nitrosylation of Drp1 mediates betaamyloid-related mitochondrial fission and neuronal injury. Science 324: 102-105, 2009.

57. Choi J, Ravipati A, Nimmagadda V, Schubert M, Castellani RJ, and Russell JW. Potential roles of PINK1 for increased PGC-1alpha-mediated mitochondrial fatty acid oxidation and their associations with Alzheimer disease and diabetes. Mitochondrion 18: 41-48, 2014.

58. Cholerton B, Baker LD, and Craft S. Insulin, cognition, and dementia. Eur J Pharmacol 719: 170-179, 2013. 
59. Chou JL, Shenoy DV, Thomas N, Choudhary PK, LaFerla FM, Goodman SR, and Breen GA. Early dysregulation of the mitochondrial proteome in a mouse model of Alzheimer's disease. J Proteomics 74: 466-479, 2011.

60. Chowdhury SKR, Smith DR, Saleh A, Schapansky J, Marquez A, Gomes S, Akude E, Morrow D, Calcutt NA, and Fernyhough P. Impaired adenosine monophosphateactivated protein kinase signalling in dorsal root ganglia neurons is linked to mitochondrial dysfunction and peripheral neuropathy in diabetes. Brain 135: 1751-1766, 2012.

61. Clarke JR, Lyra ESNM, Figueiredo CP, Frozza RL, Ledo JH, Beckman D, Katashima CK, Razolli D, Carvalho BM, Frazao R, Silveira MA, Ribeiro FC, Bomfim TR, Neves FS, Klein WL, Medeiros R, LaFerla FM, Carvalheira JB, Saad MJ, Munoz DP, Velloso LA, Ferreira ST, and De Felice FG. Alzheimer-associated Abeta oligomers impact the central nervous system to induce peripheral metabolic deregulation. EMBO Mol Med 7: 190-210, 2015.

62. Congdon EE and Sigurdsson EM. Tau-targeting therapies for Alzheimer disease. Nat Rev Neurol 14: 399-415, 2018.

63. Corder EH, Saunders AM, Risch NJ, Strittmatter WJ, Schmechel DE, Gaskell PC, Jr., Rimmler JB, Locke PA, Conneally PM, Schmader KE, Small GW, Roses AD, Haines JL, and Pericak-Vance MA. Protective effect of apolipoprotein E type 2 allele for late onset Alzheimer disease. Nat Genet 7: 180-184, 1994.

64. Corder EH, Saunders AM, Strittmatter WJ, Schmechel DE, Gaskell PC, Small GW, Roses AD, Haines JL, and Pericak-Vance MA. Gene dose of apolipoprotein E type 4 allele and the risk of Alzheimer's disease in late onset families. Science 261: 921-923, 1993.

65. Cotman CW and Berchtold NC. Exercise: a behavioral intervention to enhance brain health and plasticity. Trends Neurosci 25: 295-301, 2002.

66. Craft S. The role of metabolic disorders in Alzheimer disease and vascular dementia: two roads converged. Arch Neurol 66: 300-305, 2009.

67. Craft S, Baker LD, Montine TJ, Minoshima S, Watson GS, Claxton A, Arbuckle M, Callaghan M, Tsai E, and Plymate SR. Intranasal insulin therapy for Alzheimer disease and amnestic mild cognitive impairment: a pilot clinical trial. Arch Neurol 69: 29-38, 2012.

68. This reference has been deleted.

69. Crane PK, Walker R, Hubbard RA, Li G, Nathan DM, Zheng H, Haneuse S, Craft S, Montine TJ, and Kahn SE. Glucose levels and risk of dementia. N Engl J Med 369: 540-548, 2013.

70. Cruts M, Dermaut B, Rademakers R, Broeck MVd, Stögbauer F, and Broeckhoven CV. Novel APP mutation V715A associated with presenile Alzheimer's disease in a German family. J Neurol 250: 1374-1375, 2003.

71. Cui L, Cai Y, Cheng W, Liu G, Zhao J, Cao H, Tao H, Wang Y, Yin M, and Liu T. A novel, multi-target natural drug candidate, matrine, improves cognitive deficits in Alzheimer's disease transgenic mice by inhibiting $\mathrm{A} \beta$ aggregation and blocking the RAGE/A $\beta$ axis. Mol Neurobiol 54: 1939-1952, 2017.

72. Cunnane SC, Courchesne-Loyer A, St-Pierre V, Vandenberghe C, Pierotti T, Fortier M, Croteau E, and Castellano CA. Can ketones compensate for deteriorating brain glucose uptake during aging? Implications for the risk and treatment of Alzheimer's disease. Ann N Y Acad Sci 1367: 12-20, 2016.
73. Davis JN, Hunnicutt EJ, Jr., and Chisholm JC. A mitochondrial bottleneck hypothesis of Alzheimer's disease. Mol Med Today 1: 240-247, 1995.

74. De Leo M, Borrello S, Passantino M, Palazzotti B, Mordente A, Daniele A, Filippini V, Galeotti T, and Masullo C. Oxidative stress and overexpression of manganese superoxide dismutase in patients with Alzheimer's disease. Neurosci Lett 250: 173-176, 1998.

75. De Leon MJ, Ferris SH, George AE, Christman DR, Fowler JS, Gentes C, Reisberg B, Gee B, Emmerich M, Yonekura Y, Brodie J, Kricheff II, and Wolf AP. Positron emission tomographic studies of aging and Alzheimer disease. AJNR Am J Neuroradiol 4: 568-571, 1983.

76. DeFina LF, Willis BL, Radford NB, Gao A, Leonard D, Haskell WL, Weiner MF, and Berry JD. The association between midlife cardiorespiratory fitness levels and laterlife dementia: a cohort study. Ann Intern Med 158: 162168, 2013.

77. Della Giustina A, Goldim MP, Danielski LG, Florentino D, Mathias K, Garbossa L, Oliveira Junior AN, Fileti ME, Zarbato GF, da Rosa N, Martins Laurentino AO, Fortunato JJ, Mina F, Bellettini-Santos T, Budni J, Barichello T, Dal-Pizzol F, and Petronilho F. Alpha-lipoic acid attenuates acute neuroinflammation and long-term cognitive impairment after polymicrobial sepsis. Neurochem Int 108: 436-447, 2017.

78. Devi L, Prabhu BM, Galati DF, Avadhani NG, and Anandatheerthavarada HK. Accumulation of amyloid precursor protein in the mitochondrial import channels of human Alzheimer's disease brain is associated with mitochondrial dysfunction. J Neurosci 26: 9057-9068, 2006.

79. Di Fede G, Catania M, Morbin M, Rossi G, Suardi S, Mazzoleni G, Merlin M, Giovagnoli AR, Prioni S, and Erbetta A. A recessive mutation in the APP gene with dominant-negative effect on amyloidogenesis. Science 323: 1473-1477, 2009.

80. Diaz M, Fabelo N, Martin V, Ferrer I, Gomez T, and Marin R. Biophysical alterations in lipid rafts from human cerebral cortex associate with increased BACE1/AbetaPP interaction in early stages of Alzheimer's disease. $J$ Alzheimers Dis 43: 1185-1198, 2015.

81. Dixit S, Bernardo A, Walker JM, Kennard JA, Kim GY, Kessler ES, and Harrison FE. Vitamin C deficiency in the brain impairs cognition, increases amyloid accumulation and deposition, and oxidative stress in APP/PSEN1 and normally aging mice. ACS Chem Neurosci 6: 570-581, 2015.

82. Douaud G, Refsum H, de Jager CA, Jacoby R, Nichols TE, Smith SM, and Smith AD. Preventing Alzheimer's disease-related gray matter atrophy by B-vitamin treatment. Proc Natl Acad Sci U S A 110: 9523-9528, 2013.

83. Dringen R, Pawlowski PG, and Hirrlinger J. Peroxide detoxification by brain cells. J Neurosci Res 79: 157-165, 2005.

84. Du H, Guo L, Fang F, Chen D, Sosunov AA, McKhann GM, Yan Y, Wang C, Zhang H, and Molkentin JD. Cyclophilin D deficiency attenuates mitochondrial and neuronal perturbation and ameliorates learning and memory in Alzheimer's disease. Nat Med 14: 1097-1105, 2008.

85. Du H, Guo L, Yan S, Sosunov AA, McKhann GM, and Yan SS. Early deficits in synaptic mitochondria in an Alzheimer's disease mouse model. Proc Natl Acad Sci U S A 107: 18670-18675, 2010.

86. Ducker GS and Rabinowitz JD. One-carbon metabolism in health and disease. Cell Metab 25: 27-42, 2017. 
87. Dumont $\mathrm{M}$ and Beal MF. Neuroprotective strategies involving ROS in Alzheimer disease. Free Radic Biol Med 51: 1014-1026, 2011.

88. Dumont M, Kipiani K, Yu F, Wille E, Katz M, Calingasan NY, Gouras GK, Lin MT, and Beal MF. Coenzyme Q10 decreases amyloid pathology and improves behavior in a transgenic mouse model of Alzheimer's disease. $J$ Alzheimers Dis 27: 211-223, 2011.

89. Dumont M, Wille E, Stack C, Calingasan NY, Beal MF, and Lin MT. Reduction of oxidative stress, amyloid deposition, and memory deficit by manganese superoxide dismutase overexpression in a transgenic mouse model of Alzheimer's disease. FASEB J 23: 2459-2466, 2009.

90. Durairajan SS, Huang YY, Yuen PY, Chen LL, Kwok KY, Liu LF, Song JX, Han QB, Xue L, Chung SK, Huang JD, Baum L, Senapati S, and Li M. Effects of HuanglianJie-Du-Tang and its modified formula on the modulation of amyloid-beta precursor protein processing in Alzheimer's disease models. PLoS One 9: e92954, 2014.

91. Durairajan SS, Liu LF, Lu JH, Chen LL, Yuan Q, Chung SK, Huang L, Li XS, Huang JD, and Li M. Berberine ameliorates beta-amyloid pathology, gliosis, and cognitive impairment in an Alzheimer's disease transgenic mouse model. Neurobiol Aging 33: 2903-2919, 2012.

92. Eckert A, Keil U, Scherping I, Hauptmann S, and Müller WE. Stabilization of mitochondrial membrane potential and improvement of neuronal energy metabolism by Ginkgo biloba extract EGb 761. Ann N Y Acad Sci 1056: 474-485, 2005.

93. Eckert A, Steiner B, Marques C, Leutz S, Romig H, Haass $\mathrm{C}$, and Müller WE. Elevated vulnerability to oxidative stress-induced cell death and activation of caspase- 3 by the Swedish amyloid precursor protein mutation. $\mathrm{J} \mathrm{Neu}$ rosci Res 64: 183-192, 2001.

94. Eckman CB, Mehta ND, Crook R, Perez-tur J, Prihar G, Pfeiffer E, Graff-Radford N, Hinder P, Yager D, and Zenk B. A new pathogenic mutation in the APP gene (I716V) increases the relative proportion of A $\beta 42$ (43). Hum Mol Genet 6: 2087-2089, 1997.

95. Edland SD. Insulin-degrading enzyme, apolipoprotein E, and Alzheimer's disease. J Mol Neurosci 23: 213-217, 2004.

96. Egan JJ, Greenberg AS, Chang MK, Wek SA, Moos MC, Jr., and Londos C. Mechanism of hormone-stimulated lipolysis in adipocytes: translocation of hormone-sensitive lipase to the lipid storage droplet. Proc Natl Acad Sci U S A 89: 8537-8541, 1992.

97. Engelhart MJ, Geerlings MI, Ruitenberg A, van Swieten JC, Hofman A, Witteman JC, and Breteler MM. Dietary intake of antioxidants and risk of Alzheimer disease. JAMA 287: 3223-3229, 2002.

98. Erickson KI, Voss MW, Prakash RS, Basak C, Szabo A, Chaddock L, Kim JS, Heo S, Alves H, and White SM. Exercise training increases size of hippocampus and improves memory. Proc Natl Acad Sci U S A 108: 30173022, 2011.

99. Evans AR, Gu L, Guerrero R, and Robinson RA. Global cPILOT analysis of the APP/PS-1 mouse liver proteome. Proteomics Clin Appl 9: 872-884, 2015.

100. Fabelo N, Martin V, Marin R, Moreno D, Ferrer I, and Diaz M. Altered lipid composition in cortical lipid rafts occurs at early stages of sporadic Alzheimer's disease and facilitates APP/BACE1 interactions. Neurobiol Aging 35: 1801-1812, 2014.
101. Fabelo N, Martin V, Marin R, Santpere G, Aso E, Ferrer I, and Diaz M. Evidence for premature lipid raft aging in APP/ PS1 double-transgenic mice, a model of familial Alzheimer disease. J Neuropathol Exp Neurol 71: 868-881, 2012.

102. Farmer BC, Kluemper J, and Johnson LA. Apolipoprotein E4 alters astrocyte fatty acid metabolism and lipid droplet formation. Cells 8: pii: E182, 2019.

103. Farooqui AA, Liss L, and Horrocks LA. Stimulation of lipolytic enzymes in Alzheimer's disease. In: Lipid Storage Disorders, edited by Salvayre R, Douste-Blazy L, and Gatt S. Boston, MA: Springer, 1988, pp. 689-698.

104. Farris W, Mansourian S, Chang Y, Lindsley L, Eckman EA, Frosch MP, Eckman CB, Tanzi RE, Selkoe DJ, and Guénette $\mathrm{S}$. Insulin-degrading enzyme regulates the levels of insulin, amyloid $\beta$-protein, and the $\beta$-amyloid precursor protein intracellular domain in vivo. Proc Natl Acad Sci U S A 100: 4162-4167, 2003.

105. Farris W, Mansourian S, Leissring MA, Eckman EA, Bertram L, Eckman CB, Tanzi RE, and Selkoe DJ. Partial loss-of-function mutations in insulin-degrading enzyme that induce diabetes also impair degradation of amyloid beta-protein. Am J Pathol 164: 1425-1434, 2004.

106. Feng Z, Liu Z, Li X, Jia H, Sun L, Tian C, Jia L, and Liu J. $\alpha$-Tocopherol is an effective Phase II enzyme inducer: protective effects on acrolein-induced oxidative stress and mitochondrial dysfunction in human retinal pigment epithelial cells. J Nutr Biochem 21: 1222-1231, 2010.

107. Filadi R, Greotti E, Turacchio G, Luini A, Pozzan T, and Pizzo P. Presenilin 2 modulates endoplasmic reticulummitochondria coupling by tuning the antagonistic effect of mitofusin 2. Cell Rep 15: 2226-2238, 2016.

108. Foley P. Lipids in Alzheimer's disease: a century-old story. Biochim Biophys Acta 1801: 750-753, 2010.

109. Freund-Levi Y, Eriksdotter-Jonhagen M, Cederholm T, Basun H, Faxen-Irving G, Garlind A, Vedin I, Vessby B, Wahlund LO, and Palmblad J. Omega-3 fatty acid treatment in 174 patients with mild to moderate Alzheimer disease: OmegAD study: a randomized double-blind trial. Arch Neurol 63: 1402-1408, 2006.

110. Friedman JR and Nunnari J. Mitochondrial form and function. Nature 505: 335-343, 2014.

111. Frigerio CS, Lau P, Troakes C, Deramecourt V, Gele P, Van Loo P, Voet T, and De Strooper B. On the identification of low allele frequency mosaic mutations in the brains of Alzheimer's disease patients. Alzheimers Dement 11: 1265-1276, 2015.

112. Frisardi V, Solfrizzi V, Capurso C, Imbimbo BP, Vendemiale G, Seripa D, Pilotto A, and Panza F. Is insulin resistant brain state a central feature of the metabolic-cognitive syndrome? J Alzheimers Dis 21: 5763, 2010.

113. Gaiteri C, Mostafavi S, Honey CJ, De Jager PL, and Bennett DA. Genetic variants in Alzheimer diseasemolecular and brain network approaches. Nat Rev Neurol 12: 413-427, 2016.

114. Galasko DR, Peskind E, Clark CM, Quinn JF, Ringman JM, Jicha GA, Cotman C, Cottrell B, Montine TJ, Thomas RG, and Aisen P. Antioxidants for Alzheimer disease: a randomized clinical trial with cerebrospinal fluid biomarker measures. Arch Neurol 69: 836-841, 2012.

115. Gao F, Zhang YF, Zhang ZP, Fu LA, Cao XL, Zhang YZ, Guo CJ, Yan XC, Yang QC, Hu YY, Zhao XH, Wang YZ, Wu SX, Ju G, Zheng MH, and Han H. miR-342-5p regulates neural stem cell proliferation and differentiation 
downstream to notch signaling in mice. Stem Cell Reports 8: 1032-1045, 2017.

116. Gao $\mathrm{X}$ and Talalay $\mathrm{P}$. Induction of phase 2 genes by sulforaphane protects retinal pigment epithelial cells against photooxidative damage. Proc Natl Acad Sci U S A 101: 10446-10451, 2004.

117. Gao X and Tang XC. Huperzine A attenuates mitochondrial dysfunction in $\beta$-amyloid-treated PC12 cells by reducing oxygen free radicals accumulation and improving mitochondrial energy metabolism. J Neurosci Res 83: 1048-1057, 2006.

118. Gao X, Zheng CY, Yang L, Tang XC, and Zhang HY. Huperzine A protects isolated rat brain mitochondria against $\beta$-amyloid peptide. Free Radic Biol Med 46: 1454-1462, 2009.

119. Gasior M, Rogawski MA, and Hartman AL. Neuroprotective and disease-modifying effects of the ketogenic diet. Behav Pharmacol 17: 431-439, 2006.

120. Gatta L, Cardinale A, Wannenes F, Consoli C, Armani A, Molinari F, Mammi C, Stocchi F, Torti M, and Rosano GM. Peripheral blood mononuclear cells from mild cognitive impairment patients show deregulation of Bax and Sod1 mRNAs. Neurosci Lett 453: 36-40, 2009.

121. Ghidoni R, Albertini V, Squitti R, Paterlini A, Bruno A, Bernardini S, Cassetta E, Rossini PM, Squitieri F, and Benussi L. Novel T719P A $\beta$ PP mutation unbalances the relative proportion of amyloid- $\beta$ peptides. $J$ Alzheimers Dis 18: 295-303, 2009.

122. Ghosh S, Castillo E, Frias ES, and Swanson RA. Bioenergetic regulation of microglia. Glia 66: 1200-1212, 2018.

123. Gibson G, Haroutunian V, Zhang H, Park L, Shi Q, Lesser M, Mohs R, Sheu R, and Blass J. Mitochondrial damage in Alzheimer's disease varies with apolipoprotein E genotype. Ann Neurol 48: 297-303, 2000.

124. Gibson G, Sheu K-F, and Blass J. Abnormalities of mitochondrial enzymes in Alzheimer disease. $J$ Neural Transm 105: 855-870, 1998.

125. Goate A, Chartier-Harlin M-C, Mullan M, Brown J, Crawford F, Fidani L, Giuffra L, Haynes A, Irving N, and James L. Segregation of a missense mutation in the amyloid precursor protein gene with familial Alzheimer's disease. Nature 349: 704, 1991.

126. González-Domínguez R, García-Barrera T, Vitorica J, and Gómez-Ariza JL. High throughput multiorgan metabolomics in the APP/PS1 mouse model of Alzheimer's disease. Electrophoresis 36: 2237-2249, 2015.

127. Gonzalez-Dominguez R, García-Barrera T, Vitorica J, and Gómez-Ariza JL. Metabolomic investigation of systemic manifestations associated with Alzheimer's disease in the APP/PS1 transgenic mouse model. Mol Biosyst 11: 2429 2440, 2015.

128. Grabowski TJ, Cho HS, Vonsattel JPG, Rebeck GW, and Greenberg SM. Novel amyloid precursor protein mutation in an Iowa family with dementia and severe cerebral amyloid angiopathy. Ann Neurol 49: 697-705, 2001.

129. Gray LR, Tompkins SC, and Taylor EB. Regulation of pyruvate metabolism and human disease. Cell Mol Life Sci 71: 2577-2604, 2014.

130. Green DJ, Dawson EA, Groenewoud HM, Jones H, and Thijssen DH. Is flow-mediated dilation nitric oxide mediated?: a meta-analysis. Hypertension 63: 376-382, 2014.

131. Green DJ, Jones H, Thijssen D, Cable NT, and Atkinson G. Flow-mediated dilation and cardiovascular event pre- diction: does nitric oxide matter? Hypertension 57: 363369, 2011.

132. Grimm MO, Mett J, Stahlmann CP, Haupenthal VJ, Blumel T, Stotzel H, Grimm HS, and Hartmann T. Eicosapentaenoic acid and docosahexaenoic acid increase the degradation of amyloid-beta by affecting insulindegrading enzyme. Biochem Cell Biol 94: 534-542, 2016.

133. Grossi C, Rigacci S, Ambrosini S, Dami TE, Luccarini I, Traini C, Failli P, Berti A, Casamenti F, and Stefani M. The polyphenol oleuropein aglycone protects TgCRND8 mice against Aß plaque pathology. PLoS One 8: e71702, 2013.

134. Grundke-Iqbal I, Iqbal $\mathrm{K}$, Tung YC, Quinlan $\mathrm{M}$, Wisniewski HM, and Binder LI. Abnormal phosphorylation of the microtubule-associated protein tau (tau) in Alzheimer cytoskeletal pathology. Proc Natl Acad Sci U S A 83: 4913-4917, 1986.

135. Gu J, Jin N, Ma D, Chu D, Iqbal K, Gong CX, and Liu F. Calpain I activation causes GLUT3 proteolysis and downregulation of O-GlcNAcylation in Alzheimer's disease brain. J Alzheimers Dis 62: 1737-1746, 2018.

136. Gu L, Evans AR, and Robinson RA. Sample multiplexing with cysteine-selective approaches: cysDML and cPILOT. J Am Soc Mass Spectrom 26: 615-630, 2015.

137. Gu L and Guo Z. Alzheimer's $\mathrm{A} \beta 42$ and $\mathrm{A} \beta 40$ peptides form interlaced amyloid fibrils. J Neurochem 126: 305$311,2013$.

138. Guerreiro R, Wojtas A, Bras J, Carrasquillo M, Rogaeva E, Majounie E, Cruchaga C, Sassi C, Kauwe JS, and Younkin S. TREM2 variants in Alzheimer's disease. $N$ Engl J Med 368: 117, 2013.

139. Guerreiro RJ, Baquero M, Blesa R, Boada M, Brás JM, Bullido MJ, Calado A, Crook R, Ferreira C, and Frank A. Genetic screening of Alzheimer's disease genes in Iberian and African samples yields novel mutations in presenilins and APP. Neurobiol Aging 31: 725-731, 2010.

140. Guo J, Cheng J, North BJ, and Wei W. Functional analyses of major cancer-related signaling pathways in Alzheimer's disease etiology. Biochim Biophys Acta Rev Cancer 1868: 341-358, 2017.

141. Guo R, Zong S, Wu M, Gu J, and Yang M. Architecture of human mitochondrial respiratory megacomplex I2III2IV2. Cell 170: 1247-1257.e12, 2017.

142. Gusdon AM, Callio J, Distefano G, O'Doherty RM, Goodpaster BH, Coen PM, and Chu CT. Exercise increases mitochondrial complex I activity and DRP1 expression in the brains of aged mice. Exp Gerontol 90: 113, 2017

143. Haferlach T, Nagata Y, Grossmann V, Okuno Y, Bacher U, Nagae G, Schnittger S, Sanada M, Kon A, Alpermann T, Yoshida K, Roller A, Nadarajah N, Shiraishi Y, Shiozawa Y, Chiba K, Tanaka H, Koeffler HP, Klein HU, Dugas M, Aburatani H, Kohlmann A, Miyano S, Haferlach C, Kern W, and Ogawa S. Landscape of genetic lesions in 944 patients with myelodysplastic syndromes. Leukemia 28: 241-247, 2014.

144. Hagen TM, Ingersoll RT, Wehr CM, Lykkesfeldt J, Vinarsky V, Bartholomew JC, Song MH, and Ames BN. Acetyl-L-carnitine fed to old rats partially restores mitochondrial function and ambulatory activity. Proc Natl Acad Sci U S A 95: 9562-9566, 1998.

145. Hager K, Kenklies M, McAfoose J, Engel J, and Münch G. $\alpha$-Lipoic acid as a new treatment option for Alzheimer's disease: a 48 months follow-up analysis. J Neural Transm 189-193, 2007. 
146. Hakansson K, Ledreux A, Daffner $\mathrm{K}$, Terjestam $\mathrm{Y}$, Bergman P, Carlsson R, Kivipelto M, Winblad B, Granholm AC, and Mohammed AK. BDNF responses in healthy older persons to 35 minutes of physical exercise, cognitive training, and mindfulness: associations with working memory function. J Alzheimers Dis 55: 645-657, 2017.

147. Halagappa VK, Guo Z, Pearson M, Matsuoka Y, Cutler RG, Laferla FM, and Mattson MP. Intermittent fasting and caloric restriction ameliorate age-related behavioral deficits in the triple-transgenic mouse model of Alzheimer's disease. Neurobiol Dis 26: 212-220, 2007.

148. Halle A, Hornung V, Petzold GC, Stewart CR, Monks BG, Reinheckel T, Fitzgerald KA, Latz E, Moore KJ, and Golenbock DT. The NALP3 inflammasome is involved in the innate immune response to amyloid-beta. Nat Immunol 9: 857-865, 2008.

149. Hao J, Shen W, Sun L, Long J, Sharman E, Shi X, and Liu J. Mitochondrial dysfunction in the liver of type 2 diabetic Goto-Kakizaki rats: improvement by a combination of nutrients. Br J Nutr 106: 648-655, 2011.

150. Hao J, Shen W, Tian C, Liu Z, Ren J, Luo C, Long J, Sharman E, and Liu J. Mitochondrial nutrients improve immune dysfunction in the type 2 diabetic Goto-Kakizaki rats. J Cell Mol Med 13: 701-711, 2009.

151. Hardy J and Selkoe DJ. The amyloid hypothesis of Alzheimer's disease: progress and problems on the road to therapeutics. Science 297: 353-356, 2002.

152. Harold D, Abraham R, Hollingworth P, Sims R, Gerrish A, Hamshere ML, Pahwa JS, Moskvina V, Dowzell K, Williams A, Jones N, Thomas C, Stretton A, Morgan AR, Lovestone S, Powell J, Proitsi P, Lupton MK, Brayne C, Rubinsztein DC, Gill M, Lawlor B, Lynch A, Morgan K, Brown KS, Passmore PA, Craig D, McGuinness B, Todd S, Holmes C, Mann D, Smith AD, Love S, Kehoe PG, Hardy J, Mead S, Fox N, Rossor M, Collinge J, Maier W, Jessen F, Schurmann B, Heun R, van den Bussche H, Heuser I, Kornhuber J, Wiltfang J, Dichgans M, Frolich L, Hampel H, Hull M, Rujescu D, Goate AM, Kauwe JS, Cruchaga C, Nowotny P, Morris JC, Mayo K, Sleegers K, Bettens K, Engelborghs S, De Deyn PP, Van Broeckhoven C, Livingston G, Bass NJ, Gurling $\mathrm{H}$, McQuillin A, Gwilliam R, Deloukas P, Al-Chalabi A, Shaw CE, Tsolaki M, Singleton AB, Guerreiro R, Muhleisen TW, Nothen MM, Moebus S, Jockel KH, Klopp N, Wichmann HE, Carrasquillo MM, Pankratz VS, Younkin SG, Holmans PA, O’Donovan M, Owen MJ, and Williams J. Genomewide association study identifies variants at CLU and PICALM associated with Alzheimer's disease. Nat Genet 41: 1088-1093, 2009.

153. Hastings NB, Wang X, Song L, Butts BD, Grotz D, Hargreaves R, Fred Hess J, Hong KK, Huang CR, Hyde L, Laverty M, Lee J, Levitan D, Lu SX, Maguire M, Mahadomrongkul V, McEachern EJ, Ouyang X, Rosahl TW, Selnick H, Stanton M, Terracina G, Vocadlo DJ, Wang G, Duffy JL, Parker EM, and Zhang L. Inhibition of O-GlcNAcase leads to elevation of O-GlcNAc tau and reduction of tauopathy and cerebrospinal fluid tau in rTg4510 mice. Mol Neurodegener 12: 39, 2017.

154. Hendriks L, Van Duijn CM, Cras P, Cruts M, Van Hul W, Van Harskamp F, Warren A, McInnis MG, Antonarakis SE, and Martin J-J. Presenile dementia and cerebral haemorrhage linked to a mutation at codon 692 of the $\beta$-amyloid precursor protein gene. Nat Genet 1: 218, 1992.
155. Heneka MT, Kummer MP, Stutz A, Delekate A, Schwartz S, Vieira-Saecker A, Griep A, Axt D, Remus A, Tzeng TC, Gelpi E, Halle A, Korte M, Latz E, and Golenbock DT. NLRP3 is activated in Alzheimer's disease and contributes to pathology in APP/PS1 mice. Nature 493: 674678, 2013.

156. Herzig MC, Winkler DT, Burgermeister P, Pfeifer M, Kohler E, Schmidt SD, Danner S, Abramowski D, Sturchler-Pierrat C, Burki K, van Duinen SG, MaatSchieman ML, Staufenbiel M, Mathews PM, and Jucker M. Abeta is targeted to the vasculature in a mouse model of hereditary cerebral hemorrhage with amyloidosis. Nat Neurosci 7: 954-960, 2004.

157. Hirai K, Aliev G, Nunomura A, Fujioka H, Russell RL, Atwood CS, Johnson AB, Kress Y, Vinters HV, and Tabaton M. Mitochondrial abnormalities in Alzheimer's disease. J Neurosci 21: 3017-3023, 2001.

158. Hodson R. Alzheimer's disease. Nature 559: S1, 2018.

159. Hoffmann K, Sobol NA, Frederiksen KS, Beyer N, Vogel A, Vestergaard K, Braendgaard H, Gottrup H, Lolk A, Wermuth L, Jacobsen S, Laugesen LP, Gergelyffy RG, Hogh P, Bjerregaard E, Andersen BB, Siersma V, Johannsen P, Cotman CW, Waldemar G, and Hasselbalch SG. Moderate-to-high intensity physical exercise in patients with Alzheimer's disease: a randomized controlled trial. J Alzheimers Dis 50: 443-453, 2016.

160. Hollingworth P, Harold D, Sims R, Gerrish A, Lambert JC, Carrasquillo MM, Abraham R, Hamshere ML, Pahwa JS, Moskvina V, Dowzell K, Jones N, Stretton A, Thomas C, Richards A, Ivanov D, Widdowson C, Chapman J, Lovestone S, Powell J, Proitsi P, Lupton MK, Brayne C, Rubinsztein DC, Gill M, Lawlor B, Lynch A, Brown KS, Passmore PA, Craig D, McGuinness B, Todd S, Holmes C, Mann D, Smith AD, Beaumont H, Warden D, Wilcock G, Love S, Kehoe PG, Hooper NM, Vardy ER, Hardy J, Mead S, Fox NC, Rossor M, Collinge J, Maier W, Jessen F, Ruther E, Schurmann B, Heun R, Kolsch H, van den Bussche H, Heuser I, Kornhuber J, Wiltfang J, Dichgans M, Frolich L, Hampel H, Gallacher J, Hull M, Rujescu D, Giegling I, Goate AM, Kauwe JS, Cruchaga C, Nowotny P, Morris JC, Mayo K, Sleegers K, Bettens K, Engelborghs S, De Deyn PP, Van Broeckhoven C, Livingston G, Bass NJ, Gurling H, McQuillin A, Gwilliam R, Deloukas P, Al-Chalabi A, Shaw CE, Tsolaki M, Singleton $\mathrm{AB}$, Guerreiro R, Muhleisen TW, Nothen MM, Moebus S, Jockel KH, Klopp N, Wichmann HE, Pankratz VS, Sando SB, Aasly JO, Barcikowska M, Wszolek ZK, Dickson DW, Graff-Radford NR, Petersen RC, van Duijn CM, Breteler MM, Ikram MA, DeStefano AL, Fitzpatrick AL, Lopez O, Launer LJ, Seshadri S, Berr C, Campion D, Epelbaum J, Dartigues JF, Tzourio C, Alperovitch A, Lathrop M, Feulner TM, Friedrich P, Riehle C, Krawczak M, Schreiber S, Mayhaus M, Nicolhaus S, Wagenpfeil S, Steinberg S, Stefansson H, Stefansson K, Snaedal J, Bjornsson S, Jonsson PV, Chouraki V, Genier-Boley B, Hiltunen M, Soininen H, Combarros O, Zelenika D, Delepine M, Bullido MJ, Pasquier F, Mateo I, Frank-Garcia A, Porcellini E, Hanon O, Coto E, Alvarez V, Bosco P, Siciliano G, Mancuso M, Panza F, Solfrizzi V, Nacmias B, Sorbi S, Bossu P, Piccardi P, Arosio B, Annoni G, Seripa D, Pilotto A, Scarpini E, Galimberti D, Brice A, Hannequin D, Licastro F, Jones L, Holmans PA, Jonsson $\mathrm{T}$, Riemenschneider M, Morgan K, Younkin SG, Owen MJ, O'Donovan M, Amouyel P, and Williams J. Common 
variants at $\mathrm{ABCA} 7, \mathrm{MS} 4 \mathrm{~A} 6 \mathrm{~A} / \mathrm{MS} 4 \mathrm{~A} 4 \mathrm{E}, \mathrm{EPHA} 1, \mathrm{CD} 33$ and CD2AP are associated with Alzheimer's disease. Nat Genet 43: 429-435, 2011.

161. Honea R, Thomas GP, Harsha A, Anderson HS, Donnelly JE, Brooks WM, and Burns JM. Cardiorespiratory fitness and preserved medial temporal lobe volume in Alzheimer's disease. Alzheimer Dis Assoc Disord 23: 188-197, 2009.

162. Honig LS, Vellas B, Woodward M, Boada M, Bullock R, Borrie M, Hager K, Andreasen N, Scarpini E, Liu-Seifert H, Case M, Dean RA, Hake A, Sundell K, Poole Hoffmann V, Carlson C, Khanna R, Mintun M, DeMattos R, Selzler KJ, and Siemers E. Trial of Solanezumab for mild dementia due to Alzheimer's disease. N Engl J Med 378: 321-330, 2018.

163. Hou C, Peng Y, Qin C, Fan F, Liu J, and Long J. Hydrogen-rich water improves cognitive impairment gender-dependently in APP/PS1 mice without affecting A $\beta$ clearance. Free Radic Res 52: 1311-1322, 2018.

164. Hou C, Wang Y, Zhu E, Yan C, Zhao L, Wang X, Qiu Y, Shen H, Sun X, Feng Z, Liu J, and Long J. Coral calcium hydride prevents hepatic steatosis in high fat diet-induced obese rats: A potent mitochondrial nutrient and phase II enzyme inducer. Biochem Pharmacol 103: 85-97, 2016.

165. Hou Z, Qin X, Hu Y, Zhang X, Li G, Wu J, Li J, Sha J, Chen J, Xia J, Wang L, and Gao F. Longterm exercisederived exosomal miR-342-5p. Circ Res 124: 1386-1400, 2019.

166. Hoyer S. Abnormalities of glucose metabolism in Alzheimer's disease. Ann N Y Acad Sci 640: 53-58, 1991.

167. Hroudová J, Singh N, and Fišar Z. Mitochondrial dysfunctions in neurodegenerative diseases: relevance to Alzheimer's disease. Biomed Res Int 2014: 175062, 2014.

168. Hsu S, Gordon BA, Hornbeck R, Norton JB, Levitch D, Louden A, Ziegemeier E, Laforce R, Chhatwal J, and Day GS. Discovery and validation of autosomal dominant Alzheimer's disease mutations. Alzheimers Res Ther 10: 67, 2018.

169. Huang HC, Xu K, and Jiang ZF. Curcumin-mediated neuroprotection against amyloid-beta-induced mitochondrial dysfunction involves the inhibition of GSK-3beta. J Alzheimers Dis 32: 981-996, 2012.

170. Huang M, Chen S, Liang Y, and Guo Y. The role of berberine in the multi-target treatment of senile dementia. Curr Top Med Chem 16: 867-873, 2016.

171. Hudson G, Sims R, Harold D, Chapman J, Hollingworth P, Gerrish A, Russo G, Hamshere M, Moskvina V, Jones N, Thomas C, Stretton A, Holmans PA, O'Donovan MC, Owen MJ, Williams J, and Chinnery PF. No consistent evidence for association between mtDNA variants and Alzheimer disease. Neurology 78: 1038-1042, 2012.

172. Hyman BT and Tanzi R. Molecular epidemiology of Alzheimer's disease. $N$ Engl J Med 333: 1281-1284, 1995.

173. Imamura K, Takeshima T, Kashiwaya Y, Nakaso K, and Nakashima K. D- $\beta$-hydroxybutyrate protects dopaminergic SH-SY5Y cells in a rotenone model of Parkinson's disease. J Neurosci Res 84: 1376-1384, 2006.

174. Intlekofer KA and Cotman CW. Exercise counteracts declining hippocampal function in aging and Alzheimer's disease. Neurobiol Dis 57: 47-55, 2013.

175. Isobe $\mathrm{C}, \mathrm{Abe} \mathrm{T}$, and Terayama $\mathrm{Y}$. Levels of reduced and oxidized coenzyme Q-10 and 8-hydroxy-2'deoxyguanosine in the CSF of patients with Alzheimer's disease demonstrate that mitochondrial oxidative damage and/or oxidative DNA damage contributes to the neurodegenerative process. J Neurol 257: 399-404, 2010.

176. Itzykson R, Kosmider O, Renneville A, Gelsi-Boyer V, Meggendorfer $\mathrm{M}$, Morabito $\mathrm{M}$, Berthon $\mathrm{C}$, Ades L, Fenaux P, Beyne-Rauzy O, Vey N, Braun T, Haferlach T, Dreyfus F, Cross NC, Preudhomme C, Bernard OA, Fontenay M, Vainchenker W, Schnittger S, Birnbaum D, Droin N, and Solary E. Prognostic score including gene mutations in chronic myelomonocytic leukemia. J Clin Oncol 31: 2428-2436, 2013.

177. Jack CR, Jr., Bennett DA, Blennow K, Carrillo MC, Dunn B, Haeberlein SB, Holtzman DM, Jagust W, Jessen F, Karlawish J, Liu E, Molinuevo JL, Montine T, Phelps C, Rankin KP, Rowe CC, Scheltens P, Siemers E, Snyder HM, and Sperling R. NIA-AA Research Framework: toward a biological definition of Alzheimer's disease. Alzheimers Dement 14: 535-562, 2018.

178. Jagust WJ, Seab JP, Huesman RH, Valk PE, Mathis CA, Reed BR, Coxson PG, and Budinger TF. Diminished glucose transport in Alzheimer's disease: dynamic PET studies. J Cereb Blood Flow Metab 11: 323-330, 1991.

179. Janssen J, Beck J, Campbell T, Dickinson A, Fox N, Harvey R, Houlden H, Rossor M, and Collinge J. Early onset familial Alzheimer's disease: mutation frequency in 31 families. Neurology 60: 235-239, 2003.

180. Jeong HS, Park JS, Yang Y, Na SH, Chung YA, and Song IU. Cerebral perfusion changes after acetyl-L-carnitine treatment in early Alzheimer's disease using single photon emission computed tomography. Dement Neurocogn Disord 16: 26-31, 2017.

181. Jia J, Wang F, Wei C, Zhou A, Jia X, Li F, Tang M, Chu L, Zhou Y, Zhou C, Cui Y, Wang Q, Wang W, Yin P, Hu N, Zuo X, Song H, Qin W, Wu L, Li D, Jia L, Song J, Han Y, Xing Y, Yang P, Li Y, Qiao Y, Tang Y, Lv J, and Dong $X$. The prevalence of dementia in urban and rural areas of China. Alzheimers Dement 10: 1-9, 2014.

182. Jin Q, Jiang S, Wu Y-L, Bai T, Yang Y, Jin X, Lian L-H, and Nan J-X. Hepatoprotective effect of cryptotanshinone from Salvia miltiorrhiza in D-galactosamine/ lipopolysaccharide-induced fulminant hepatic failure. Phytomedicine 21: 141-147, 2014.

183. Johansson H, Kanis JA, Odén A, McCloskey E, Chapurlat RD, Christiansen C, Cummings SR, Diez-Perez A, Eisman JA, and Fujiwara S. A meta-analysis of the association of fracture risk and body mass index in women. J Bone Miner Res 29: 223-233, 2014.

184. Johnson LA, Torres ER, Impey S, Stevens JF, and Raber J. Apolipoprotein E4 and insulin resistance interact to impair cognition and alter the epigenome and metabolome. Sci Rep 7: 43701, 2017.

185. Johnson LA, Torres ER, Weber Boutros S, Patel E, Akinyeke T, Alkayed NJ, and Raber J. Apolipoprotein E4 mediates insulin resistance-associated cerebrovascular dysfunction and the post-prandial response. J Cereb Blood Flow Metab 39: 770-781, 2019.

186. Joseph J, Shukitt-Hale B, Denisova NA, Martin A, Perry $\mathrm{G}$, and Smith MA. Copernicus revisited: amyloid beta in Alzheimer's disease. Neurobiol Aging 22: 131-146, 2001.

187. Kaden D, Harmeier A, Weise C, Munter LM, Althoff V, Rost BR, Hildebrand PW, Schmitz D, Schaefer M, and Lurz R. Novel APP/A $\beta$ mutation K16N produces highly toxic heteromeric A $\beta$ oligomers. EMBO Mol Med 4: 647659, 2012. 
188. Kamino K, Orr HT, Payami H, Wijsman EM, Alonso ME, Pulst SM, Anderson L, O'dahl S, Nemens E, and White JA. Linkage and mutational analysis of familial Alzheimer disease kindreds for the APP gene region. Am J Hum Genet 51: 998, 1992.

189. Kandimalla R, Manczak M, Fry D, Suneetha Y, Sesaki H, and Reddy PH. Reduced dynamin-related protein 1 protects against phosphorylated Tau-induced mitochondrial dysfunction and synaptic damage in Alzheimer's disease. Hum Mol Genet 25: 4881-4897, 2016.

190. Kandimalla R, Manczak M, Yin X, Wang R, and Reddy $\mathrm{PH}$. Hippocampal phosphorylated tau induced cognitive decline, dendritic spine loss and mitochondrial abnormalities in a mouse model of Alzheimer's disease. Hum Mol Genet 27: 30-40, 2018.

191. Kang E-B, Kwon I-S, Koo J-H, Kim E-J, Kim C-H, Lee J, Yang C-H, Lee Y-I, Cho I-H, and Cho J-Y. Treadmill exercise represses neuronal cell death and inflammation during $\mathrm{A} \beta$-induced $\mathrm{ER}$ stress by regulating unfolded protein response in aged presenilin 2 mutant mice. Apoptosis 18: 1332-1347, 2013.

192. Kang EB and Cho JY. Effect of treadmill exercise on PI3K/ AKT/mTOR, autophagy, and Tau hyperphosphorylation in the cerebral cortex of NSE/htau23 transgenic mice. J Exerc Nutrition Biochem 19: 199-209, 2015.

193. Kashiwaya Y, Takeshima T, Mori N, Nakashima K, Clarke K, and Veech RL. D- $\beta$-Hydroxybutyrate protects neurons in models of Alzheimer's and Parkinson's disease. Proc Natl Acad Sci U S A 97: 5440-5444, 2000.

194. Kawai D, Takaki A, Nakatsuka A, Wada J, Tamaki N, Yasunaka T, Koike K, Tsuzaki R, Matsumoto K, and Miyake Y. Hydrogen-rich water prevents progression of nonalcoholic steatohepatitis and accompanying hepatocarcinogenesis in mice. Hepatology 56: 912-921, 2012.

195. Keaney $J$ and Campbell $M$. The dynamic blood-brain barrier. FEBS J 282: 4067-4079, 2015.

196. Kelleher RJ and Soiza RL. Evidence of endothelial dysfunction in the development of Alzheimer's disease: is Alzheimer's a vascular disorder? Am J Cardiovasc Dis 3: 197-226, 2013.

197. Kempuraj D, Thangavel R, Selvakumar GP, Zaheer S, Ahmed ME, Raikwar SP, Zahoor H, Saeed D, Natteru PA, and Iyer S. Brain and peripheral atypical inflammatory mediators potentiate neuroinflammation and neurodegeneration. Front Cell Neurosci 11: 216, 2017.

198. Kerr JS, Adriaanse BA, Greig NH, Mattson MP, Cader MZ, Bohr VA, and Fang EF. Mitophagy and Alzheimer's disease: cellular and molecular mechanisms. Trends Neurosci 40: 151-166, 2017.

199. Kim HS, Kim MK, Lee M, Kwon BS, Suh DH, and Song YS. Effect of red ginseng on genotoxicity and healthrelated quality of life after adjuvant chemotherapy in patients with epithelial ovarian cancer: a randomized, double blind, placebo-controlled trial. Nutrients 9: pii: E772, 2017.

200. Kim JH, Kim M, He XB, Wulansari N, Yoon BH, Bae DH, Huh N, Kim YS, Lee SH, and Kim SY. Vitamin C promotes astrocyte differentiation through DNA hydroxymethylation. Stem Cells 36: 1578-1588, 2018.

201. Kivipelto M, Helkala EL, Laakso MP, Hanninen T, Hallikainen M, Alhainen K, Soininen H, Tuomilehto J, and Nissinen A. Midlife vascular risk factors and Alzheimer's disease in later life: longitudinal, population based study. BMJ 322: 1447-1451, 2001.
202. Klein CP, Hoppe JB, Saccomori AB, Dos Santos BG, Sagini JP, Crestani MS, August PM, Hozer RM, Grings M, Parmeggiani B, Leipnitz G, Navas P, Salbego CG, and Matte C. Physical exercise during pregnancy prevents cognitive impairment induced by amyloid-beta in adult offspring rats. Mol Neurobiol 56: 2022-2038, 2019.

203. Koo JH, Kwon IS, Kang EB, Lee CK, Lee NH, Kwon MG, Cho IH, and Cho JY. Neuroprotective effects of treadmill exercise on BDNF and PI3-K/Akt signaling pathway in the cortex of transgenic mice model of Alzheimer's disease. J Exerc Nutrition Biochem 17: 151-160, 2013.

204. Kopeikina KJ, Carlson GA, Pitstick R, Ludvigson AE, Peters A, Luebke JI, Koffie RM, Frosch MP, Hyman BT, and Spires-Jones TL. Tau accumulation causes mitochondrial distribution deficits in neurons in a mouse model of tauopathy and in human Alzheimer's disease brain. Am J Pathol 179: 2071-2082, 2011.

205. Kossoff EH. More fat and fewer seizures: dietary therapies for epilepsy. Lancet Neurol 3: 415-420, 2004.

206. Kotsiou K, Tasioula-Margari M, Capuano E, and Fogliano V. Effect of standard phenolic compounds and olive oil phenolic extracts on acrylamide formation in an emulsion system. Food Chem 124: 242-247, 2011.

207. Kritchevsky D and Moyer AW. Anti-cholesterol activity of alpha-lipoic acid. Nature 182: 396, 1958.

208. Kroeze LI, van der Reijden BA, and Jansen JH. 5-Hydroxymethylcytosine: an epigenetic mark frequently deregulated in cancer. Biochim Biophys Acta 1855: 144154, 2015.

209. Kumar DKV, Choi SH, Washicosky KJ, Eimer WA, Tucker S, Ghofrani J, Lefkowitz A, McColl G, Goldstein LE, and Tanzi RE. Amyloid- $\beta$ peptide protects against microbial infection in mouse and worm models of Alzheimer's disease. Sci Transl Med 8: 340ra72, 2016.

210. Kumar-Singh S, De Jonghe C, Cruts M, Kleinert R, Wang R, Mercken M, De Strooper B, Vanderstichele H, Löfgren A, and Vanderhoeven I. Nonfibrillar diffuse amyloid deposition due to a $\gamma 42$-secretase site mutation points to an essential role for N-truncated A $\beta 42$ in Alzheimer's disease. Hum Mol Genet 9: 2589-2598, 2000.

211. Kwok JB, Li QX, Hallupp M, Whyte S, Ames D, Beyreuther K, Masters CL, and Schofield PR. Novel Leu723Pro amyloid precursor protein mutation increases amyloid $\beta 42$ (43) peptide levels and induces apoptosis. Ann Neurol 47: 249-253, 2000.

212. LaDu MJ, Falduto MT, Manelli AM, Reardon CA, Getz GS, and Frail DE. Isoform-specific binding of apolipoprotein E to beta-amyloid. J Biol Chem 269: 2340323406, 1994.

213. Lambert JC, Heath S, Even G, Campion D, Sleegers K, Hiltunen M, Combarros O, Zelenika D, Bullido MJ, Tavernier B, Letenneur L, Bettens K, Berr C, Pasquier F, Fievet N, Barberger-Gateau P, Engelborghs S, De Deyn P, Mateo I, Franck A, Helisalmi S, Porcellini E, Hanon O, de Pancorbo MM, Lendon C, Dufouil C, Jaillard C, Leveillard T, Alvarez V, Bosco P, Mancuso M, Panza F, Nacmias B, Bossu P, Piccardi P, Annoni G, Seripa D, Galimberti D, Hannequin D, Licastro F, Soininen H, Ritchie K, Blanche H, Dartigues JF, Tzourio C, Gut I, Van Broeckhoven C, Alperovitch A, Lathrop M, and Amouyel P. Genome-wide association study identifies variants at CLU and CR1 associated with Alzheimer's disease. Nat Genet 41: 1094-1099, 2009. 
214. Larner A. Presenilin-1 mutations in Alzheimer's disease: an update on genotype-phenotype relationships. $J$ Alzheimers Dis 37: 653-659, 2013.

215. Lazar AN, Bich C, Panchal M, Desbenoit N, Petit VW, Touboul D, Dauphinot L, Marquer C, Laprevote O, Brunelle A, and Duyckaerts C. Time-of-flight secondary ion mass spectrometry (TOF-SIMS) imaging reveals cholesterol overload in the cerebral cortex of Alzheimer disease patients. Acta Neuropathol 125: 133-144, 2013.

216. Leal NS, Schreiner B, Pinho CM, Filadi R, Wiehager B, Karlstrom H, Pizzo P, and Ankarcrona M. Mitofusin-2 knockdown increases ER-mitochondria contact and decreases amyloid beta-peptide production. J Cell Mol Med 20: 1686-1695, 2016.

217. Lee G, Neve RL, and Kosik KS. The microtubule binding domain of tau protein. Neuron 2: 1615-1624, 1989.

218. Lee HM, Lee OH, Kim KJ, and Lee BY. Ginsenoside Rg1 promotes glucose uptake through activated AMPK pathway in insulin-resistant muscle cells. Phytother Res 26: 1017-1022, 2012.

219. Leem Y-H, Lee Y-I, Son H-J, and Lee S-H. Chronic exercise ameliorates the neuroinflammation in mice carrying NSE/htau23. Biochem Biophys Res Commun 406: 359365, 2011.

220. Leutner S, Schindowski K, Frölich L, Maurer K, Kratzsch T, Eckert A, and Müller WE. Enhanced ROS-generation in lymphocytes from Alzheimer's patients. Pharmacopsychiatry 38: 312-315, 2005.

221. Levy E, Carman MD, Fernandez-Madrid IJ, Power MD, Lieberburg I, van Duinen SG, Bots G, Luyendijk W, and Frangione B. Mutation of the Alzheimer's disease amyloid gene in hereditary cerebral hemorrhage, Dutch type. Science 248: 1124-1126, 1990.

222. Li FJ, Shen L, and Ji HF. Dietary intakes of vitamin E, vitamin $\mathrm{C}$, and beta-carotene and risk of Alzheimer's disease: a meta-analysis. J Alzheimers Dis 31: 253-258, 2012.

223. Li J, Wang C, Zhang JH, Cai J-M, Cao Y-P, and Sun X-J. Hydrogen-rich saline improves memory function in a rat model of amyloid-beta-induced Alzheimer's disease by reduction of oxidative stress. Brain Res 1328: 152-161, 2010.

224. Li XH, Lv BL, Xie JZ, Liu J, Zhou XW, and Wang JZ. AGEs induce Alzheimer-like tau pathology and memory deficit via RAGE-mediated GSK-3 activation. Neurobiol Aging 33: 1400-1410, 2012.

225. Li Z, Okamoto K-I, Hayashi Y, and Sheng M. The importance of dendritic mitochondria in the morphogenesis and plasticity of spines and synapses. Cell 119: 873-887, 2004.

226. Lim WL, Martins IJ, and Martins RN. The involvement of lipids in Alzheimer's disease. J Genet Genomics 41: 261274, 2014.

227. Lin MT and Beal MF. Mitochondrial dysfunction and oxidative stress in neurodegenerative diseases. Nature 443: 787-795, 2006.

228. Liu F, Iqbal K, Grundke-Iqbal I, and Gong CX. Involvement of aberrant glycosylation in phosphorylation of tau by cdk5 and GSK-3beta. FEBS Lett 530: 209-214, 2002.

229. Liu F, Iqbal K, Grundke-Iqbal I, Hart GW, and Gong C-X. O-GlcNAcylation regulates phosphorylation of tau: a mechanism involved in Alzheimer's disease. Proc Natl Acad Sci U S A 101: 10804-10809, 2004.
230. Liu F, Shi J, Tanimukai H, Gu J, Gu J, Grundke-Iqbal I, Iqbal K, and Gong CX. Reduced O-GlcNAcylation links lower brain glucose metabolism and tau pathology in Alzheimer's disease. Brain 132: 1820-1832, 2009.

231. Liu H, Rose ME, Culver S, Ma X, Dixon CE, and Graham $\mathrm{SH}$. Rosiglitazone attenuates inflammation and CA3 neuronal loss following traumatic brain injury in rats. Biochem Biophys Res Commun 472: 648-655, 2016.

232. Liu H-L, Zhao G, and Zhang H. Long-term treadmill exercise inhibits the progression of Alzheimer's diseaselike neuropathology in the hippocampus of APP/PS1 transgenic mice. Behav Brain Res 256: 261-272, 2013.

233. Liu J. The effects and mechanisms of mitochondrial nutrient $\alpha$-lipoic acid on improving age-associated mitochondrial and cognitive dysfunction: an overview. Neurochem Res 33: 194-203, 2008.

234. Liu J and Ames BN. Reducing mitochondrial decay with mitochondrial nutrients to delay and treat cognitive dysfunction, Alzheimer's disease, and Parkinson's disease. Nutr Neurosci 8: 67-89, 2005.

235. Liu J, Head E, Gharib AM, Yuan W, Ingersoll RT, Hagen TM, Cotman CW, and Ames BN. Memory loss in old rats is associated with brain mitochondrial decay and RNA/ DNA oxidation: partial reversal by feeding acetyl-Lcarnitine and/or R- $\alpha$-lipoic acid. Proc Natl Acad Sci U S A 99: 2356-2361, 2002.

236. Liu J, Killilea DW, and Ames BN. Age-associated mitochondrial oxidative decay: improvement of carnitine acetyltransferase substrate-binding affinity and activity in brain by feeding old rats acetyl-L-carnitine and/or R- $\alpha$-lipoic acid. Proc Natl Acad Sci U S A 99: 1876-1881, 2002.

237. Liu J, Peng Y, Feng Z, Shi W, Qu L, Li Y, Liu J, and Long J. Reloading functionally ameliorates disuse-induced muscle atrophy by reversing mitochondrial dysfunction, and similar benefits are gained by administering a combination of mitochondrial nutrients. Free Radic Biol Med 69: 116-128, 2014.

238. Liu J, Peng Y, Wang X, Fan Y, Qin C, Shi L, Tang Y, Cao $\mathrm{K}, \mathrm{Li} \mathrm{H}$, and Long J. Mitochondrial dysfunction launches dexamethasone-induced skeletal muscle atrophy via AMPK/FOXO3 signaling. Mol Pharm 13: 73-84, 2015.

239. Liu J, Shen W, Zhao B, Wang Y, Wertz K, Weber P, and Zhang $\mathrm{P}$. Targeting mitochondrial biogenesis for preventing and treating insulin resistance in diabetes and obesity: hope from natural mitochondrial nutrients. Adv Drug Deliv Rev 61: 1343-1352, 2009.

240. Liu Y, Cheng A, Li YJ, Yang Y, Kishimoto Y, Zhang S, Wang Y, Wan R, Raefsky SM, Lu D, Saito T, Saido T, Zhu J, Wu LJ, and Mattson MP. SIRT3 mediates hippocampal synaptic adaptations to intermittent fasting and ameliorates deficits in APP mutant mice. Nat Commun 10: 1886, 2019.

241. Long J, Gao F, Tong L, Cotman CW, Ames BN, and Liu J. Mitochondrial decay in the brains of old rats: ameliorating effect of alpha-lipoic acid and acetyl-L-carnitine. Neurochem Res 34: 755-763, 2009.

242. Long J, He P, Shen Y, and Li R. New evidence of mitochondria dysfunction in the female Alzheimer's disease brain: deficiency of estrogen receptor- $\beta$. J Alzheimers Dis 30: 545-558, 2012.

243. Lopez-Lopez C, Dietrich MO, Metzger F, Loetscher H, and Torres-Aleman I. Disturbed cross talk between insulin-like growth factor I and AMP-activated protein kinase as a possible cause of vascular dysfunction in the 
amyloid precursor protein/presenilin 2 mouse model of Alzheimer's disease. J Neurosci 27: 824-831, 2007.

244. Lovell MA, Ehmann WD, Butler SM, and Markesbery WR. Elevated thiobarbituric acid-reactive substances and antioxidant enzyme activity in the brain in Alzheimer's disease. Neurology 45: 1594-1601, 1995.

245. Lovell MA, Xie C, Xiong S, and Markesbery WR. Protection against amyloid beta peptide and iron/hydrogen peroxide toxicity by alpha lipoic acid. $J$ Alzheimers Dis 5: 229-239, 2003.

246. Luchsinger JA, Reitz C, Honig LS, Tang MX, Shea S, and Mayeux R. Aggregation of vascular risk factors and risk of incident Alzheimer disease. Neurology 65: 545-551, 2005.

247. Lunnon K, Ibrahim Z, Proitsi P, Lourdusamy A, Newhouse S, Sattlecker M, Furney S, Saleem M, Soininen H, Kloszewska I, Mecocci P, Tsolaki M, Vellas B, Coppola G, Geschwind D, Simmons A, Lovestone S, Dobson R, and Hodges A. Mitochondrial dysfunction and immune activation are detectable in early Alzheimer's disease blood. J Alzheimers Dis 30: 685-710, 2012.

248. Lustbader JW, Cirilli M, Lin C, Xu HW, Takuma K, Wang N, Caspersen C, Chen X, Pollak S, and Chaney M. ABAD directly links $A ß$ to mitochondrial toxicity in Alzheimer's disease. Science 304: 448-452, 2004.

249. This reference has been deleted.

250. Lv J, Ma S, Zhang X, Zheng L, Ma Y, Zhao X, Lai W, Shen H, Wang Q, and Ji J. Quantitative proteomics reveals that PEA15 regulates astroglial $\mathrm{A} \beta$ phagocytosis in an Alzheimer's disease mouse model. J Proteomics 110: 4558, 2014.

251. Maalouf M, Rho JM, and Mattson MP. The neuroprotective properties of calorie restriction, the ketogenic diet, and ketone bodies. Brain Res Rev 59: 293-315, 2009.

252. Maalouf M, Sullivan PG, Davis L, Kim DY, and Rho JM. Ketones inhibit mitochondrial production of reactive oxygen species production following glutamate excitotoxicity by increasing NADH oxidation. Neuroscience 145: 256-264, 2007.

253. Magistretti PJ. Imaging brain aerobic glycolysis as a marker of synaptic plasticity. Proc Natl Acad Sci U S A 113: 7015-7016, 2016.

254. Magistretti PJ and Allaman I. Lactate in the brain: from metabolic end-product to signalling molecule. Nat Rev Neurosci 19: 235-249, 2018.

255. Maione F, Piccolo M, De Vita S, Chini MG, Cristiano C, De Caro C, Lippiello P, Miniaci MC, Santamaria R, Irace C, De Feo V, Calignano A, Mascolo N, and Bifulco G. Down regulation of pro-inflammatory pathways by tanshinone IIA and cryptotanshinone in a non-genetic mouse model of Alzheimer's disease. Pharmacol Res 129: 482-490, 2018.

256. Mancuso M, Orsucci D, LoGerfo A, Calsolaro V, and Siciliano G. Clinical features and pathogenesis of Alzheimer's disease: involvement of mitochondria and mitochondrial DNA. In: Diseases of DNA Repair, edited by Ahmad SI. New York, NY: Springer, 2010, pp. 34-44.

257. Manczak M, Anekonda TS, Henson E, Park BS, Quinn J, and Reddy $\mathrm{PH}$. Mitochondria are a direct site of $\mathrm{A} \beta$ accumulation in Alzheimer's disease neurons: implications for free radical generation and oxidative damage in disease progression. Hum Mol Genet 15: 1437-1449, 2006.

258. Manczak M, Calkins MJ, and Reddy PH. Impaired mitochondrial dynamics and abnormal interaction of amyloid beta with mitochondrial protein Drp1 in neurons from patients with Alzheimer's disease: implications for neuronal damage. Hum Mol Genet 20: 2495-2509, 2011.

259. Manczak M, Park BS, Jung Y, and Reddy PH. Differential expression of oxidative phosphorylation genes in patients with Alzheimer's disease. Neuromolecular Med 5: 147162, 2004.

260. Manczak M and Reddy PH. Abnormal interaction between the mitochondrial fission protein Drp1 and hyperphosphorylated tau in Alzheimer's disease neurons: implications for mitochondrial dysfunction and neuronal damage. Hum Mol Genet 21: 2538-2547, 2012.

261. Mangialasche F, Solomon A, Winblad B, Mecocci P, and Kivipelto M. Alzheimer's disease: clinical trials and drug development. Lancet Neurol 9: 702-716, 2010.

262. Mano $Y$, Kotani $T$, Ito $M$, Nagai $T$, Ichinohashi $Y$, Yamada K, Ohno K, Kikkawa F, and Toyokuni S. Maternal molecular hydrogen administration ameliorates rat fetal hippocampal damage caused by in utero ischemiareperfusion. Free Radic Biol Med 69: 324-330, 2014.

263. Manolopoulos K, Klotz L, Korsten P, Bornstein S, and Barthel A. Linking Alzheimer's disease to insulin resistance: the FoxO response to oxidative stress. Mol Psychiatry 15: 1046-1052, 2010.

264. Mapstone M, Cheema AK, Fiandaca MS, Zhong X, Mhyre TR, MacArthur LH, Hall WJ, Fisher SG, Peterson DR, and Haley JM. Plasma phospholipids identify antecedent memory impairment in older adults. Nat Med 20: 415-418, 2014.

265. Markesbery WR, Kryscio RJ, Lovell MA, and Morrow JD. Lipid peroxidation is an early event in the brain in amnestic mild cognitive impairment. Ann Neurol 58: 730$735,2005$.

266. Martinez-Reyes I, Diebold LP, Kong H, Schieber M, Huang H, Hensley CT, Mehta MM, Wang T, Santos JH, Woychik R, Dufour E, Spelbrink JN, Weinberg SE, Zhao Y, DeBerardinis RJ, and Chandel NS. TCA cycle and mitochondrial membrane potential are necessary for diverse biological functions. Molecular cell 61: 199-209, 2016.

267. Masino SA, Li T, Theofilas P, Sandau US, Ruskin DN, Fredholm BB, Geiger JD, Aronica E, and Boison D. A ketogenic diet suppresses seizures in mice through adenosine A 1 receptors. J Clin Invest 121: 2679-2683, 2011.

268. Mast N, Saadane A, Valencia-Olvera A, Constans J, Maxfield E, Arakawa H, Li Y, Landreth G, and Pikuleva IA. Cholesterol-metabolizing enzyme cytochrome P450 46A1 as a pharmacologic target for Alzheimer's disease. Neuropharmacology 123: 465-476, 2017.

269. McManus MJ, Murphy MP, and Franklin JL. The mitochondria-targeted antioxidant MitoQ prevents loss of spatial memory retention and early neuropathology in a transgenic mouse model of Alzheimer's disease. J Neurosci 31: 15703-15715, 2011.

270. Mecocci P, MacGarvey U, and Beal MF. Oxidative damage to mitochondrial DNA is increased in Alzheimer's disease. Ann Neurol 36: 747-751, 1994.

271. Mei Z, Zhang F, Tao L, Zheng W, Cao Y, Wang Z, Tang S, Le K, Chen S, and Pi R. Cryptotanshinone, a compound from Salvia miltiorrhiza modulates amyloid precursor protein metabolism and attenuates $\beta$-amyloid deposition through upregulating $\alpha$-secretase in vivo and in vitro. Neurosci Lett 452: 90-95, 2009.

272. Minoshima S, Giordani B, Berent S, Frey KA, Foster NL, and Kuhl DE. Metabolic reduction in the posterior cin- 
gulate cortex in very early Alzheimer's disease. Ann Neurol 42: 85-94, 1997.

273. Mody N, Agouni A, McIlroy G, Platt B, and Delibegovic M. Susceptibility to diet-induced obesity and glucose intolerance in the APP SWE/PSEN1 A246E mouse model of Alzheimer's disease is associated with increased brain levels of protein tyrosine phosphatase 1B (PTP1B) and retinol-binding protein 4 (RBP4), and basal phosphorylation of S6 ribosomal protein. Diabetologia 54: 2143 2151, 2011.

274. Montesanto A, Crocco P, Anfossi M, Smirne N, Puccio G, Colao R, Maletta R, Passarino G, Bruni AC, and Rose $\mathrm{G}$. The genetic variability of UCP4 affects the individual susceptibility to late-onset Alzheimer's disease and modifies the disease's risk in APOE-varepsilon4 carriers. J Alzheimers Dis 51: 1265-1274, 2016.

275. Moreira PI, Harris PL, Zhu X, Santos MS, Oliveira CR, Smith MA, and Perry G. Lipoic acid and N-acetyl cysteine decrease mitochondrial-related oxidative stress in Alzheimer disease patient fibroblasts. J Alzheimers Dis 12: 195-206, 2007.

276. Moreira PI, Santos MS, and Oliveira CR. Alzheimer's disease: a lesson from mitochondrial dysfunction. Antioxid Redox Signal 9: 1621-1630, 2007.

277. Morel M, Authelet M, Dedecker R, and Brion JP. Glycogen synthase kinase-3beta and the p25 activator of cyclin dependent kinase 5 increase pausing of mitochondria in neurons. Neuroscience 167: 1044-1056, 2010.

278. Morris MC, Evans DA, Bienias JL, Tangney CC, Bennett DA, Wilson RS, Aggarwal N, and Schneider J. Consumption of fish and n-3 fatty acids and risk of incident Alzheimer disease. Arch Neurol 60: 940-946, 2003.

279. Mosconi L. Brain glucose metabolism in the early and specific diagnosis of Alzheimer's disease. Eur J Nucl Med Mol Imaging 32: 486-510, 2005.

280. Mosconi L, Mistur R, Switalski R, Tsui WH, Glodzik L, Li Y, Pirraglia E, De Santi S, Reisberg B, Wisniewski T, and de Leon MJ. FDG-PET changes in brain glucose metabolism from normal cognition to pathologically verified Alzheimer's disease. Eur J Nucl Med Mol Imaging 36: 811-822, 2009.

281. Mosconi L, Pupi A, and De Leon MJ. Brain glucose hypometabolism and oxidative stress in preclinical Alzheimer's disease. Ann N Y Acad Sci 1147: 180-195, 2008.

282. Mota SI, Costa RO, Ferreira IL, Santana I, Caldeira GL, Padovano C, Fonseca AC, Baldeiras I, Cunha C, Letra L, Oliveira CR, Pereira CM, and Rego AC. Oxidative stress involving changes in Nrf2 and ER stress in early stages of Alzheimer's disease. Biochim Biophys Acta 1852: 14281441, 2015.

283. Mullan M, Crawford F, Axelman K, Houlden H, Lilius L, Winblad B, and Lannfelt L. A pathogenic mutation for probable Alzheimer's disease in the APP gene at the Nterminus of $\beta$-amyloid. Nat Genet 1: 345, 1992.

284. Murrell J, Farlow M, Ghetti B, and Benson MD. A mutation in the amyloid precursor protein associated with hereditary Alzheimer's disease. Science 254: 97-99, 1991.

285. Murrell JR, Hake AM, Quaid KA, Farlow MR, and Ghetti B. Early-onset Alzheimer disease caused by a new mutation (V717L) in the amyloid precursor protein gene. Arch Neurol 57: 885-887, 2000.

286. Muthukumaran K, Kanwar A, Vegh C, Marginean A, Elliott A, Guilbeault N, Badour A, Sikorska M, Cohen J, and Pandey S. Ubisol-Q10 (a nanomicellar water-soluble formulation of CoQ10) treatment inhibits Alzheimer-type behavioral and pathological symptoms in a double transgenic mouse (TgAPEswe, PSEN1dE9) model of Alzheimer's disease. J Alzheimers Dis 61: 221-236, 2018.

287. Naderi J, Lopez C, and Pandey S. Chronically increased oxidative stress in fibroblasts from Alzheimer's disease patients causes early senescence and renders resistance to apoptosis by oxidative stress. Mech Ageing Dev 127: 25$35,2006$.

288. Nagpal R, Neth BJ, Wang S, Craft S, and Yadav H. Modified Mediterranean-ketogenic diet modulates gut microbiome and short-chain fatty acids in association with Alzheimer's disease markers in subjects with mild cognitive impairment. EBioMedicine 47: 529-542, 2019.

289. Naudi A, Cabre R, Jove M, Ayala V, Gonzalo H, PorteroOtin M, Ferrer I, and Pamplona R. Lipidomics of human brain aging and Alzheimer's disease pathology. Int Rev Neurobiol 122: 133-189, 2015.

290. Neeper SA, Gomez-Pinilla F, Choi J, and Cotman C. Exercise and brain neurotrophins. Nature 373: 109, 1995.

291. Newman JC and Verdin E. Ketone bodies as signaling metabolites. Trends Endocrinol Metab 25: 42-52, 2014.

292. Ng LF, Gruber J, Cheah IK, Goo CK, Cheong WF, Shui G, Sit KP, Wenk MR, and Halliwell B. The mitochondriatargeted antioxidant MitoQ extends lifespan and improves healthspan of a transgenic Caenorhabditis elegans model of Alzheimer disease. Free Radic Biol Med 71: 390-401, 2014.

293. Ng T, Liu F, and Wang Z. Antioxidative activity of natural products from plants. Life Sci 66: 709-723, 2000.

294. Niccoli T, Cabecinha M, Tillmann A, Kerr F, Wong CT, Cardenes D, Vincent AJ, Bettedi L, Li L, Gronke S, Dols $\mathrm{J}$, and Partridge L. Increased glucose transport into neurons rescues Abeta toxicity in drosophila. Curr Biol 26: 2291-2300, 2016.

295. Nicolas G, Wallon D, Charbonnier C, Quenez O, Rousseau S, Richard A-C, Rovelet-Lecrux A, Coutant S, Le Guennec K, and Bacq D. Screening of dementia genes by whole-exome sequencing in early-onset Alzheimer disease: input and lessons. Eur J Hum Genet 24: 710, 2016.

296. Nigam SM, Xu S, Kritikou JS, Marosi K, Brodin L, and Mattson MP. Exercise and BDNF reduce Abeta production by enhancing alpha-secretase processing of APP. J Neurochem 142: 286-296, 2017.

297. Obici L, Demarchi A, De Rosa G, Bellotti V, Marciano S, Donadei S, Arbustini E, Palladini G, Diegoli M, and Genovese E. A novel A $\beta$ PP mutation exclusively associated with cerebral amyloid angiopathy. Ann Neurol 58: 639-644, 2005.

298. Ohara T, Doi Y, Ninomiya T, Hirakawa Y, Hata J, Iwaki T, Kanba S, and Kiyohara Y. Glucose tolerance status and risk of dementia in the community The Hisayama Study. Neurology 77: 1126-1134, 2011.

299. Ohsawa I, Ishikawa M, Takahashi K, Watanabe M, Nishimaki K, Yamagata K, Katsura K-I, Katayama Y, Asoh S, and Ohta S. Hydrogen acts as a therapeutic antioxidant by selectively reducing cytotoxic oxygen radicals. Nat Med 13: 688-694, 2007.

300. Pamplona R, Dalfo E, Ayala V, Bellmunt MJ, Prat J, Ferrer I, and Portero-Otin M. Proteins in human brain cortex are modified by oxidation, glycoxidation, and lipoxidation. Effects of Alzheimer disease and identification of lipoxidation targets. J Biol Chem 280: 2152221530, 2005. 
301. Pan X, Niu G, and Liu H. Microwave-assisted extraction of tanshinones from Salvia miltiorrhiza bunge with analysis by high-performance liquid chromatography. J Chromatogr A 922: 371-375, 2001.

302. Panchal M, Loeper J, Cossec JC, Perruchini C, Lazar A, Pompon D, and Duyckaerts C. Enrichment of cholesterol in microdissected Alzheimer's disease senile plaques as assessed by mass spectrometry. J Lipid Res 51: 598-605, 2010.

303. Parks JK, Smith TS, Trimmer PA, Bennett JP, Jr., and Parker WD, Jr. Neurotoxic Abeta peptides increase oxidative stress in vivo through NMDA-receptor and nitricoxide-synthase mechanisms, and inhibit complex IV activity and induce a mitochondrial permeability transition in vitro. J Neurochem 76: 1050-1056, 2001.

304. Pasalar P, Najmabadi H, Noorian A, Moghimi B, Jannati A, Soltanzadeh A, Krefft T, Crook R, and Hardy J. An Iranian family with Alzheimer's disease caused by a novel APP mutation (Thr714Ala). Neurology 58: 1574-1575, 2002.

305. Pastorino L, Sun A, Lu PJ, Zhou XZ, Balastik M, Finn G, Wulf G, Lim J, Li SH, Li X, Xia W, Nicholson LK, and $\mathrm{Lu}$ KP. The prolyl isomerase Pin1 regulates amyloid precursor protein processing and amyloid-beta production. Nature 440: 528-534, 2006.

306. Peacock ML, Murman DL, Sima AA, Warren J, Jr., Roses $\mathrm{AD}$, and Fink JK. Novel amyloid precursor protein gene mutation (codon 665Asp) in a patient with late-onset Alzheimer's disease. Ann Neurol 35: 432-438, 1994.

307. Peng Y, Hou C, Yang Z, Li C, Jia L, Liu J, Tang Y, Shi L, Li Y, and Long J. Hydroxytyrosol mildly improve cognitive function independent of APP processing in APP/ PS1 mice. Mol Nutr Food Res 60: 2331-2342, 2016.

308. Peng Y, Liu J, Shi L, Tang Y, Gao D, Long J, and Liu J. Mitochondrial dysfunction precedes depression of AMPK/AKT signaling in insulin resistance induced by high glucose in primary cortical neurons. $J$ Neurochem 137: 701-713, 2016.

309. Peng Y, Liu J, Tang Y, Liu J, Han T, Han S, Li H, Hou C, Liu J, and Long J. High-fat-diet-induced weight gain ameliorates bone loss without exacerbating A $\beta \mathrm{PP}$ processing and cognition in female APP/PS1 mice. Front Cell Neurosci 8: 1-14, 2014.

310. Perez-Gracia E, Torrejon-Escribano B, and Ferrer I. Dystrophic neurites of senile plaques in Alzheimer's disease are deficient in cytochrome c oxidase. Acta Neuropathol 116: 261-268, 2008.

311. Pericak-Vance MA and Haines JL. Genetic susceptibility to Alzheimer disease. Trends Genet 11: 504-508, 1995.

312. Pienaar IS, Howell N, and Elson JL. MutPred mutational load analysis shows mildly deleterious mitochondrial DNA variants are not more prevalent in Alzheimer's patients, but may be under-represented in healthy older individuals. Mitochondrion 34: 141-146, 2017.

313. Pietrocola F, Galluzzi L, Bravo-San Pedro JM, Madeo F, and Kroemer G. Acetyl coenzyme A: a central metabolite and second messenger. Cell Metab 21: 805-821, 2015.

314. Pinho TS, Correia SC, Perry G, Ambrosio AF, and Moreira PI. Diminished O-GlcNAcylation in Alzheimer's disease is strongly correlated with mitochondrial anomalies. Biochim Biophys Acta Mol Basis Dis 1865: 20482059, 2019.

315. Podlesniy P, Figueiro-Silva J, Llado A, Antonell A, Sanchez-Valle R, Alcolea D, Lleo A, Molinuevo JL, Serra $\mathrm{N}$, and Trullas R. Low cerebrospinal fluid concentration of mitochondrial DNA in preclinical Alzheimer disease. Ann Neurol 74: 655-668, 2013.

316. Psaltopoulou T, Sergentanis TN, Panagiotakos DB, Sergentanis IN, Kosti R, and Scarmeas N. Mediterranean diet, stroke, cognitive impairment, and depression: a metaanalysis. Ann Neurol 74: 580-591, 2013.

317. Puchalska $P$ and Crawford PA. Multi-dimensional roles of ketone bodies in fuel metabolism, signaling, and therapeutics. Cell Metab 25: 262-284, 2017.

318. Qin W, Haroutunian V, Katsel P, Cardozo CP, Ho L, Buxbaum JD, and Pasinetti GM. PGC- $1 \alpha$ expression decreases in the Alzheimer disease brain as a function of dementia. Arch Neurol 66: 352, 2009.

319. Qiu T, Liu Q, Chen Y, Zhao Y, and Li Y. A $\beta 42$ and A $\beta 40$ : similarities and differences. J Pept Sci 21: 522-529, 2015.

320. Quan Q, Wang J, Li X, and Wang Y. Ginsenoside Rg1 decreases A $\beta 1-42$ level by upregulating PPAR $\gamma$ and IDE expression in the hippocampus of a rat model of Alzheimer's disease. PLoS One 8: e59155, 2013.

321. Querfurth HW and LaFerla FM. Alzheimer's disease. $N$ Engl J Med 362: 329-344, 2010.

322. Quinn JF, Raman R, Thomas RG, Yurko-Mauro K, Nelson EB, Van Dyck C, Galvin JE, Emond J, Jack CR, Jr., Weiner M, Shinto L, and Aisen PS. Docosahexaenoic acid supplementation and cognitive decline in Alzheimer disease: a randomized trial. JAMA 304: 1903-1911, 2010.

323. Rardin MJ, Newman JC, Held JM, Cusack MP, Sorensen DJ, Li B, Schilling B, Mooney SD, Kahn CR, Verdin E, and Gibson BW. Label-free quantitative proteomics of the lysine acetylome in mitochondria identifies substrates of SIRT3 in metabolic pathways. Proc Natl Acad Sci U S A 110: 6601-6606, 2013.

324. Razmovski-Naumovski V, Huang TH-W, Tran VH, Li GQ, Duke CC, and Roufogalis BD. Chemistry and pharmacology of Gynostemma pentaphyllum. Phytochem Rev 4: 197-219, 2005.

325. Reddy JK and Hashimoto T. Peroxisomal $\beta$-oxidation and peroxisome proliferator-activated receptor $\alpha$ : an adaptive metabolic system. Annu Rev Nutr 21: 193-230, 2001.

326. Reddy PH, Mani G, Park BS, Jacques J, Murdoch G, Whetsell W, Jr., Kaye J, and Manczak M. Differential loss of synaptic proteins in Alzheimer's disease: implications for synaptic dysfunction. J Alzheimers Dis 7: 103-117, 2005.

327. Reddy PH, McWeeney S, Park BS, Manczak M, Gutala RV, Partovi D, Jung Y, Yau V, Searles R, and Mori M. Gene expression profiles of transcripts in amyloid precursor protein transgenic mice: up-regulation of mitochondrial metabolism and apoptotic genes is an early cellular change in Alzheimer's disease. Hum Mol Genet 13: 1225-1240, 2004

328. Reger MA, Watson G, Green PS, Baker LD, Cholerton B, Fishel MA, Plymate SR, Cherrier MM, Schellenberg GD, and Frey I. Intranasal insulin administration dosedependently modulates verbal memory and plasma amyloid- $\beta$ in memory-impaired older adults. $J$ Alzheimers Dis 13: 323-331, 2008.

329. Reiman EM, Caselli RJ, Yun LS, Chen K, Bandy D, Minoshima S, Thibodeau SN, and Osborne D. Preclinical evidence of Alzheimer's disease in persons homozygous for the $\varepsilon 4$ allele for apolipoprotein E. N Engl J Med 334: 752-758, 1996.

330. Reiman EM, Chen K, Alexander GE, Caselli RJ, Bandy D, Osborne D, Saunders AM, and Hardy J. Functional brain abnormalities in young adults at genetic risk for late- 
onset Alzheimer's dementia. Proc Natl Acad Sci U S A 101: 284-289, 2004.

331. Remington R, Bechtel C, Larsen D, Samar A, Doshanjh L, Fishman P, Luo Y, Smyers K, Page R, Morrell C, and Shea TB. A phase II randomized clinical trial of a nutritional formulation for cognition and mood in Alzheimer's disease. J Alzheimers Dis 45: 395-405, 2015.

332. Remington R, Bechtel C, Larsen D, Samar A, Page R, Morrell C, and Shea TB. Maintenance of cognitive performance and mood for individuals with Alzheimer's disease following consumption of a nutraceutical formulation: a one-year, open-label study. J Alzheimers Dis 51: 991-995, 2016.

333. Risner M, Saunders A, Altman J, Ormandy G, Craft S, Foley I, Zvartau-Hind M, Hosford D, and Roses A. Efficacy of rosiglitazone in a genetically defined population with mild-to-moderate Alzheimer's disease. Pharmacogenomics J 6: 246-254, 2006.

334. Roberson ED, Scearce-Levie K, Palop JJ, Yan F, Cheng IH, Wu T, Gerstein H, Yu GQ, and Mucke L. Reducing endogenous tau ameliorates amyloid beta-induced deficits in an Alzheimer's disease mouse model. Science 316: 750-754, 2007.

335. Roberts RO, Geda YE, Cerhan JR, Knopman DS, Cha RH, Christianson TJ, Pankratz VS, Ivnik RJ, Boeve BF, and O'Connor HM. Vegetables, unsaturated fats, moderate alcohol intake, and mild cognitive impairment. Dement Geriatr Cogn Disord 29: 413-423, 2010.

336. Rocchi A, Pellegrini S, Siciliano G, and Murri L. Causative and susceptibility genes for Alzheimer's disease: a review. Brain Res Bull 61: 1-24, 2003.

337. Rodríguez-Morató J, Xicota L, Fitó M, Farré M, Dierssen $\mathrm{M}$, and de la Torre R. Potential role of olive oil phenolic compounds in the prevention of neurodegenerative diseases. Molecules 20: 4655-4680, 2015.

338. Rodriguez-Rodriguez P, Sandebring-Matton A, MerinoSerrais P, Parrado-Fernandez C, Rabano A, Winblad B, Avila J, Ferrer I, and Cedazo-Minguez A. Tau hyperphosphorylation induces oligomeric insulin accumulation and insulin resistance in neurons. Brain 140: 3269-3285, 2017.

339. Saido $\mathrm{T}$ and Leissring MA. Proteolytic degradation of amyloid beta-protein. Cold Spring Harb Perspect Med 2: a006379, 2012.

340. Sajan M, Hansen B, Ivey R, 3rd, Sajan J, Ari C, Song S, Braun U, Leitges M, Farese-Higgs M, and Farese RV. Brain insulin signaling is increased in insulin-resistant states and decreases in FOXOs and PGC-1alpha and Increases in Abeta1-40/42 and phospho-tau may Abet Alzheimer development. Diabetes 65: 1892-1903, 2016.

341. Sano M, Bell K, Cote L, Dooneief G, Lawton A, Legler L, Marder K, Naini A, Stern Y, and Mayeux R. Double-blind parallel design pilot study of acetyl levocarnitine in patients with Alzheimer's disease. Arch Neurol 49: 11371141, 1992.

342. Sano M, Ernesto C, Thomas RG, Klauber MR, Schafer K, Grundman M, Woodbury P, Growdon J, Cotman CW, Pfeiffer E, Schneider LS, and Thal LJ. A controlled trial of selegiline, alpha-tocopherol, or both as treatment for Alzheimer's disease. The Alzheimer's disease cooperative study. N Engl J Med 336: 1216-1222, 1997.

343. Santos RX, Correia SC, Zhu X, Smith MA, Moreira PI, Castellani RJ, Nunomura A, and Perry G. Mitochondrial DNA oxidative damage and repair in aging and Alzhei- mer's disease. Antioxid Redox Signal 18: 2444-2457, 2013.

344. Sassi C, Guerreiro R, Gibbs R, Ding J, Lupton MK, Troakes C, Al-Sarraj S, Niblock M, Gallo J-M, and Adnan $\mathrm{J}$. Investigating the role of rare coding variability in Mendelian dementia genes (APP, PSEN1, PSEN2, GRN, MAPT, and PRNP) in late-onset Alzheimer's disease. Neurobiol Aging 35: 2881.e1-e2881.e6, 2014.

345. Scarmeas N, Luchsinger JA, Schupf N, Brickman AM, Cosentino S, Tang MX, and Stern Y. Physical activity, diet, and risk of Alzheimer disease. JAMA 302: 627-637, 2009.

346. Scarmeas N, Stern Y, Mayeux R, Manly JJ, Schupf N, and Luchsinger JA. Mediterranean diet and mild cognitive impairment. Arch Neurol 66: 216-225, 2009.

347. Schulte EC, Fukumori A, Mollenhauer B, Hor H, Arzberger T, Perneczky R, Kurz A, Diehl-Schmid J, Hüll M, and Lichtner P. Rare variants in $\beta$-Amyloid precursor protein (APP) and Parkinson's disease. Eur J Hum Genet 23: 1328, 2015.

348. Selkoe DJ. Alzheimer's disease is a synaptic failure. Science 298: 789-791, 2002.

349. Selkoe DJ and Hardy J. The amyloid hypothesis of Alzheimer's disease at 25 years. EMBO Mol Med 8: 595-608, 2016.

350. Seo KI, Choi MS, Jung UJ, Kim HJ, Yeo J, Jeon SM, and Lee MK. Effect of curcumin supplementation on blood glucose, plasma insulin, and glucose homeostasis related enzyme activities in diabetic db/db mice. Mol Nutr Food Res 52: 995-1004, 2008.

351. Shahpasand K, Uemura I, Saito T, Asano T, Hata K, Shibata K, Toyoshima Y, Hasegawa M, and Hisanaga $\mathrm{S}$. Regulation of mitochondrial transport and intermicrotubule spacing by tau phosphorylation at the sites hyperphosphorylated in Alzheimer's disease. J Neurosci 32: 2430-2441, 2012.

352. Shao A, Wu H, Hong Y, Tu S, Sun X, Wu Q, Zhao Q, Zhang J, and Sheng J. Hydrogen-rich saline attenuated subarachnoid hemorrhage-induced early brain injury in rats by suppressing inflammatory response: possible involvement of NF-kappaB pathway and NLRP3 inflammasome. Mol Neurobiol 53: 3462-3476, 2016.

353. Shen W, Hao J, Tian C, Ren J, Yang L, Li X, Luo C, Cotma CW, and Liu J. A combination of nutriments improves mitochondrial biogenesis and function in skeletal muscle of type 2 diabetic Goto-Kakizaki rats. PLoS One 3: e2328, 2008.

354. Sheng B, Wang X, Su B, Lee HG, Casadesus G, Perry G, and Zhu X. Impaired mitochondrial biogenesis contributes to mitochondrial dysfunction in Alzheimer's disease. J Neurochem 120: 419-429, 2012.

355. This reference has been deleted.

356. Shenk JC, Liu J, Fischbach K, Xu K, Puchowicz M, Obrenovich ME, Gasimov E, Alvarez LM, Ames BN, and LaManna JC. The effect of acetyl-L-carnitine and R- $\alpha$ lipoic acid treatment in ApoE4 mouse as a model of human Alzheimer's disease. J Neurol Sci 283: 199-206, 2009.

357. This reference has been deleted.

358. Shi C, Guo K, Yew DT, Yao Z, Forster EL, Wang H, and $\mathrm{Xu}$ J. Effects of ageing and Alzheimer's disease on mitochondrial function of human platelets. Exp Gerontol 43: 589-594, 2008.

359. Shi L, Zhao D, Hou C, Peng Y, Liu J, Zhang S, Liu J, and Long J. Early interleukin-6 enhances hepatic ketogenesis 
in APP SWE/PSEN1dE9 mice via 3-hydroxy-3methylglutary-CoA synthase 2 signaling activation by p38/nuclear factor $\kappa \mathrm{B}$ p65. Neurobiol Aging 56: 115-126, 2017.

360. Shimada K, Crother TR, Karlin J, Dagvadorj J, Chiba N, Chen S, Ramanujan VK, Wolf AJ, Vergnes L, Ojcius DM, Rentsendorj A, Vargas M, Guerrero C, Wang Y, Fitzgerald KA, Underhill DM, Town T, and Arditi M. Oxidized mitochondrial DNA activates the NLRP3 inflammasome during apoptosis. Immunity 36: 401-414, 2012.

361. Shimazu T, Hirschey MD, Hua L, Dittenhafer-Reed KE, Schwer B, Lombard DB, Li Y, Bunkenborg J, Alt FW, Denu JM, Jacobson MP, and Verdin E. SIRT3 deacetylates mitochondrial 3-hydroxy-3-methylglutaryl CoA synthase 2 and regulates ketone body production. Cell Metab 12: 654-661, 2010.

362. Shin BK, Kang S, Kim DS, and Park S. Intermittent fasting protects against the deterioration of cognitive function, energy metabolism and dyslipidemia in Alzheimer's disease-induced estrogen deficient rats. Exp Biol Med 243: 334-343, 2018.

363. Shinto L, Quinn J, Montine T, Dodge HH, Woodward W, Baldauf-Wagner S, Waichunas D, Bumgarner L, Bourdette D, Silbert L, and Kaye J. A randomized placebocontrolled pilot trial of omega-3 fatty acids and alpha lipoic acid in Alzheimer's disease. J Alzheimers Dis 38: 111-120, 2014.

364. Simpson IA, Carruthers A, and Vannucci SJ. Supply and demand in cerebral energy metabolism: the role of nutrient transporters. J Cereb Blood Flow Metab 27: 17661791, 2007.

365. Simpson IA, Chundu KR, Davies-Hill T, Honer WG, and Davies P. Decreased concentrations of GLUT1 and GLUT3 glucose transporters in the brains of patients with Alzheimer's disease. Ann Neurol 35: 546-551, 1994.

366. Singh B, Parsaik AK, Mielke MM, Erwin PJ, Knopman DS, Petersen RC, and Roberts RO. Association of mediterranean diet with mild cognitive impairment and Alzheimer's disease: a systematic review and meta-analysis. J Alzheimers Dis 39: 271-282, 2014.

367. Sleiman SF, Henry J, Al-Haddad R, El Hayek L, Abou Haidar E, Stringer T, Ulja D, Karuppagounder SS, Holson EB, Ratan RR, Ninan I, and Chao MV. Exercise promotes the expression of brain derived neurotrophic factor (BDNF) through the action of the ketone body betahydroxybutyrate. Elife 5: pii: e15092, 2016.

368. Small GW, Ercoli LM, Silverman DH, Huang S-C, Komo S, Bookheimer SY, Lavretsky H, Miller K, Siddarth P, and Rasgon NL. Cerebral metabolic and cognitive decline in persons at genetic risk for Alzheimer's disease. Proc Natl Acad Sci U S A 97: 6037-6042, 2000.

369. Small SA and Duff K. Linking Abeta and tau in late-onset Alzheimer's disease: a dual pathway hypothesis. Neuron 60: 534-542, 2008.

370. Smith GS, de Leon MJ, George AE, Kluger A, Volkow ND, McRae T, Golomb J, Ferris SH, Reisberg B, Ciaravino J, and La Regina ME. Topography of crosssectional and longitudinal glucose metabolic deficits in Alzheimer's disease. Pathophysiologic implications. Arch Neurol 49: 1142-1150, 1992.

371. Sorrentino V, Romani M, Mouchiroud L, Beck JS, Zhang H, D'amico D, Moullan N, Potenza F, Schmid AW, and
Rietsch S. Enhancing mitochondrial proteostasis reduces amyloid- $\beta$ proteotoxicity. Nature 552: 187, 2017.

372. Steen E, Terry BM, Rivera EJ, Cannon JL, Neely TR, Tavares R, Xu XJ, Wands JR, and de la Monte SM. Impaired insulin and insulin-like growth factor expression and signaling mechanisms in Alzheimer's disease-is this type 3 diabetes? J Alzheimers Dis 7: 63-80, 2005.

373. Steiner JL, Murphy EA, McClellan JL, Carmichael MD, and Davis JM. Exercise training increases mitochondrial biogenesis in the brain. J Appl Physiol 111: 1066-1071, 2011.

374. Suh JH, Shenvi SV, Dixon BM, Liu H, Jaiswal AK, Liu RM, and Hagen TM. Decline in transcriptional activity of Nrf2 causes age-related loss of glutathione synthesis, which is reversible with lipoic acid. Proc Natl Acad Sci U S A 101: 3381-3386, 2004.

375. Sultana R, Mecocci P, Mangialasche F, Cecchetti R, Baglioni M, and Butterfield DA. Increased protein and lipid oxidative damage in mitochondria isolated from lymphocytes from patients with Alzheimer's disease: insights into the role of oxidative stress in Alzheimer's disease and initial investigations into a potential biomarker for this dementing disorder. J Alzheimers Dis 24: 77-84, 2011.

376. Sun X, Wu Y, Gu M, and Zhang Y. miR-342-5p decreases ankyrin $G$ levels in Alzheimer's disease transgenic mouse models. Cell Rep 6: 264-270, 2014.

377. Swerdlow RH. Mitochondria and cell bioenergetics: increasingly recognized components and a possible etiologic cause of Alzheimer's disease. Antioxid Redox Signal 16: 1434-1455, 2012.

378. Swerdlow RH. Mitochondria and mitochondrial cascades in Alzheimer's disease. J Alzheimers Dis 62: 1403-1416, 2018.

379. Swerdlow RH, Burns JM, and Khan SM. The Alzheimer's disease mitochondrial cascade hypothesis: progress and perspectives. Biochim Biophys Acta 1842: 1219-1231, 2014.

380. Swerdlow RH and Khan SM. A "mitochondrial cascade hypothesis" for sporadic Alzheimer's disease. Med Hypotheses 63: 8-20, 2004.

381. Swerdlow RH and Khan SM. The Alzheimer's disease mitochondrial cascade hypothesis: an update. Exp Neurol 218: 308-315, 2009.

382. Tacconi S, Perri R, Balestrieri E, Grelli S, Bernardini S, Annichiarico R, Mastino A, Caltagirone C, and Macchi B. Increased caspase activation in peripheral blood mononuclear cells of patients with Alzheimer's disease. Exp Neurol 190: 254-262, 2004.

383. Tagliavini F. A new $\beta$ PP mutation related to hereditary cerebral haemorrhage. Alzheimers Rep 2: S28, 1999.

384. Takeda S, Sato N, Uchio-Yamada K, Sawada K, Kunieda T, Takeuchi D, Kurinami H, Shinohara M, Rakugi H, and Morishita R. Diabetes-accelerated memory dysfunction via cerebrovascular inflammation and $\mathrm{A} \beta$ deposition in an Alzheimer mouse model with diabetes. Proc Natl Acad Sci U S A 107: 7036-7041, 2010.

385. Talbot K, Wang H-Y, Kazi H, Han L-Y, Bakshi KP, Stucky A, Fuino RL, Kawaguchi KR, Samoyedny AJ, and Wilson RS. Demonstrated brain insulin resistance in Alzheimer's disease patients is associated with IGF-1 resistance, IRS-1 dysregulation, and cognitive decline. J Clin Invest 122: 1316-1338, 2012. 
386. Tang S, Shen X-Y, Huang H-Q, Xu S-W, Yu Y, Zhou C-H, Chen S-R, Le K, Wang Y-H, and Liu P-Q. Cryptotanshinone suppressed inflammatory cytokines secretion in RAW264. 7 macrophages through inhibition of the NF$\kappa \mathrm{B}$ and MAPK signaling pathways. Inflammation 34: 111-118, 2011.

387. Tang Y, Peng Y, Liu J, Shi L, Wang Y, Long J, and Liu J. Early inflammation-associated factors blunt sterol regulatory element-binding proteins-1-mediated lipogenesis in high-fat diet-fed APPSWE/PSEN1dE9 mouse model of Alzheimer's disease. J Neurochem 136: 791803, 2016.

388. Taylor MK, Swerdlow RH, Burns JM, and Sullivan DK. An experimental ketogenic diet for Alzheimer disease was nutritionally dense and rich in vegetables and avocado. Curr Dev Nutr 3: nzz003, 2019.

389. Teng E, Taylor K, Bilousova T, Weiland D, Pham T, Zuo X, Yang F, Chen PP, Glabe CG, Takacs A, Hoffman DR, Frautschy SA, and Cole GM. Dietary DHA supplementation in an APP/PS1 transgenic rat model of $\mathrm{AD}$ reduces behavioral and Abeta pathology and modulates Abeta oligomerization. Neurobiol Dis 82: 552-560, 2015.

390. Terni B, Boada J, Portero-Otin M, Pamplona R, and Ferrer I. Mitochondrial ATP-synthase in the entorhinal cortex is a target of oxidative stress at stages I/II of Alzheimer's disease pathology. Brain Pathol 20: 222-233, 2010.

391. Terreni L, Fogliarino S, Franceschi M, and Forloni G. Novel pathogenic mutation in an Italian patient with familial Alzheimer's disease detected in APP gene. Neurobiol Aging 23: 319, 2002.

392. Theuns J, Marjaux E, Vandenbulcke M, Van Laere K, Kumar-Singh S, Bormans G, Brouwers N, Van den Broeck M, Vennekens K, and Corsmit E. Alzheimer dementia caused by a novel mutation located in the APP C-terminal intracytosolic fragment. Hum Mutat 27: 888896, 2006.

393. Tieu K, Perier C, Caspersen C, Teismann P, Wu D-C, Yan S-D, Naini A, Vila M, Jackson-Lewis V, and Ramasamy $\mathrm{R}$. D- $\beta$-Hydroxybutyrate rescues mitochondrial respiration and mitigates features of Parkinson disease. J Clin Invest 112: 892-901, 2003.

394. Tilley L, Morgan K, and Kalsheker N. Genetic risk factors in Alzheimer's disease. Mol Pathol 51: 293-304, 1998.

395. Troesch B, Weber P, and Mohajeri MH. Potential links between impaired one-carbon metabolism due to polymorphisms, inadequate B-vitamin status, and the development of Alzheimer's disease. Nutrients 8: pii: E803, 2016.

396. Trushina E, Dutta T, Persson X-MT, Mielke MM, and Petersen RC. Identification of altered metabolic pathways in plasma and CSF in mild cognitive impairment and Alzheimer's disease using metabolomics. PLoS One 8: e63644, 2013.

397. Valla J, Schneider L, Niedzielko T, Coon KD, Caselli R, Sabbagh MN, Ahern GL, Baxter L, Alexander G, and Walker DG. Impaired platelet mitochondrial activity in Alzheimer's disease and mild cognitive impairment. $\mathrm{Mi}$ tochondrion 6: 323-330, 2006.

398. Veurink G, Liu D, Taddei K, Perry G, Smith MA, Robertson TA, Hone E, Groth DM, Atwood CS, and Martins RN. Reduction of inclusion body pathology in ApoEdeficient mice fed a combination of antioxidants. Free Radic Biol Med 34: 1070-1077, 2003.
399. Vining EP, Freeman JM, Ballaban-Gil K, Camfield CS, Camfield PR, Holmes GL, Shinnar S, Shuman R, Trevathan E, and Wheless JW. A multicenter study of the efficacy of the ketogenic diet. Arch Neurol 55: 14331437, 1998.

400. Vissing J, Andersen M, and Diemer NH. Exercise-induced changes in local cerebral glucose utilization in the rat. J Cereb Blood Flow Metab 16: 729-736, 1996.

401. von Bernhardi R and Eugenin J. Alzheimer's disease: redox dysregulation as a common denominator for diverse pathogenic mechanisms. Antioxid Redox Signal 16: 9741031, 2012.

402. von Bernhardi R, Eugenin-von Bernhardi L, and Eugenin J. Microglial cell dysregulation in brain aging and neurodegeneration. Front Aging Neurosci 7: 124, 2015.

403. Wakutani Y, Watanabe K, Adachi Y, Wada-Isoe K, Urakami K, Ninomiya H, Saido T, Hashimoto T, Iwatsubo $\mathrm{T}$, and Nakashima K. Novel amyloid precursor protein gene missense mutation (D678N) in probable familial Alzheimer's disease. J Neurol Neurosurg Psychiatry 75: 1039-1042, 2004.

404. Wallace D. Mitochondrial diseases in man and mouse. Science 283: 1482-1488, 1999.

405. Wallace DC. Mitochondrial genetics: a paradigm for aging and degenerative diseases? Science 256: 628-632, 1992.

406. Wallace DC. Mitochondria as chi. Genetics 179: 727-735, 2008.

407. Wan Z, Mah D, Simtchouk S, Kluftinger A, and Little JP. Role of amyloid $\beta$ in the induction of lipolysis and secretion of adipokines from human adipose tissue. Adipocyte 4: 212-216, 2015.

408. Wang AC, Jensen EH, Rexach JE, Vinters HV, and Hsieh-Wilson LC. Loss of O-GlcNAc glycosylation in forebrain excitatory neurons induces neurodegeneration. Proc Natl Acad Sci U S A 113: 15120-15125, 2016.

409. Wang C, Li J, Liu Q, Yang R, Zhang JH, Cao Y-P, and Sun X-J. Hydrogen-rich saline reduces oxidative stress and inflammation by inhibit of JNK and NF- $\kappa \mathrm{B}$ activation in a rat model of amyloid-beta-induced Alzheimer's disease. Neurosci Lett 491: 127-132, 2011.

410. Wang D, Calabrese EJ, Lian B, Lin Z, and Calabrese V. Hormesis as a mechanistic approach to understanding herbal treatments in traditional Chinese medicine. Pharmacol Ther 184: 42-50, 2018.

411. Wang H-M, Wang L-W, Liu X-M, Li C-L, Xu S-P, and Farooq A-D. Neuroprotective effects of forsythiaside on learning and memory deficits in senescence-accelerated mouse prone (SAMP8) mice. Pharmacol Biochem Behav 105: 134-141, 2013.

412. Wang J, Gu BJ, Masters CL, and Wang YJ. A systemic view of Alzheimer disease-insights from amyloid- $\beta$ metabolism beyond the brain. Nat Rev Neurol 13: 703, 2017.

413. Wang JZ, Grundke-Iqbal I, and Iqbal K. Glycosylation of microtubule-associated protein tau: an abnormal posttranslational modification in Alzheimer's disease. Nat Med 2: 871-875, 1996.

414. Wang P, Jia L, Chen B, Zhang L, Liu J, Long J, and Li $Y$. Hydrogen inhalation is superior to mild hypothermia in improving cardiac function and neurological outcome in an asphyxial cardiac arrest model of rats. Shock 46: 312318, 2016. 
415. Wang P, Su C, Feng H, Chen X, Dong Y, Rao Y, Ren Y, Yang J, Shi J, Tian J, and Jiang S. Curcumin regulates insulin pathways and glucose metabolism in the brains of APPswe/PS1dE9 mice. Int J Immunopathol Pharmacol 30: 25-43, 2017.

416. Wang Q, Jia J, Qin W, Wu L, Li D, Wang Q, and Li H. A novel A $\beta$ PP M722K mutation affects amyloid- $\beta$ secretion and tau phosphorylation and may cause early-onset familial Alzheimer's disease in chinese individuals. $J$ Alzheimers Dis 47: 157-165, 2015.

417. Wang W, Yin J, Ma X, Zhao F, Siedlak SL, Wang Z, Torres S, Fujioka H, Xu Y, Perry G, and Zhu X. Inhibition of mitochondrial fragmentation protects against Alzheimer's disease in rodent model. Hum Mol Genet 26: 41184131, 2017.

418. Wang X, Li H, Zheng A, Yang L, Liu J, Chen C, Tang Y, Zou X, Li Y, Long J, Zhang Y, and Feng Z. Mitochondrial dysfunction-associated OPA1 cleavage contributes to muscle degeneration: preventative effect of hydroxytyrosol acetate. Cell Death Dis 5: e1521, 2014.

419. Wang X, Su B, Lee H-G, Li X, Perry G, Smith MA, and Zhu X. Impaired balance of mitochondrial fission and fusion in Alzheimer's disease. J Neurosci 29: 9090-9103, 2009.

420. Wang X, Wang W, Li L, Perry G, Lee H-G, and Zhu $X$. Oxidative stress and mitochondrial dysfunction in Alzheimer's disease. Biochim Biophys Acta 1842: 12401247, 2014.

421. Wang Y, Viscarra J, Kim SJ, and Sul HS. Transcriptional regulation of hepatic lipogenesis. Nat Rev Mol Cell Biol 16: 678-689, 2015.

422. Wang Y, Zeng QG, Zhang ZB, Yan RM, Wang LY, and Zhu D. Isolation and characterization of endophytic huperzine A-producing fungi from Huperzia serrata. $J$ Ind Microbiol Biotechnol 38: 1267-1278, 2011.

423. Wang Z, Jackson RJ, Hong W, Taylor WM, Corbett GT, Moreno A, Liu W, Li S, Frosch MP, Slutsky I, YoungPearse TL, Spires-Jones TL, and Walsh DM. Human brain-derived Abeta oligomers bind to synapses and disrupt synaptic activity in a manner that requires APP. J Neurosci 37: 11947-11966, 2017.

424. Watson GS, Cholerton BA, Reger MA, Baker LD, Plymate SR, Asthana S, Fishel MA, Kulstad JJ, Green PS, and Cook DG. Preserved cognition in patients with early Alzheimer disease and amnestic mild cognitive impairment during treatment with rosiglitazone: a preliminary study. Am J Geriatr Psychiatry 13: 950-958, 2005.

425. WHO. Risk Reduction of Cognitive Decline and Dementia. Geneva, Switzerland: World Health Organization, 2019.

426. Willette AA, Bendlin BB, Starks EJ, Birdsill AC, Johnson SC, Christian BT, Okonkwo OC, La Rue A, Hermann BP, Koscik RL, Jonaitis EM, Sager MA, and Asthana S. Association of insulin resistance with cerebral glucose uptake in late middle-aged adults at risk for Alzheimer disease. JAMA Neurol 72: 1013-1020, 2015.

427. Winkler EA, Nishida Y, Sagare AP, Rege SV, Bell RD, Perlmutter D, Sengillo JD, Hillman S, Kong P, and Nelson AR. GLUT1 reductions exacerbate Alzheimer's disease vasculo-neuronal dysfunction and degeneration. Nat Neurosci 18: 521-530, 2015.

428. Wong MW, Braidy N, Poljak A, Pickford R, Thambisetty M, and Sachdev PS. Dysregulation of lipids in Alzhei- mer's disease and their role as potential biomarkers. Alzheimers Dement 13: 810-827, 2017.

429. Wood H. Dementia: peripheral inflammation could be a prodromal indicator of dementia. Nat Rev Neurol 14: 127, 2018.

430. World Health Organization. International Statistical Classification of Diseases and Related Health Problems. Geneva, Switzerland: World Health Organization, 2018.

431. Wrann CD, White JP, Salogiannnis J, Laznik-Bogoslavski $\mathrm{D}$, Wu J, Ma D, Lin JD, Greenberg ME, and Spiegelman BM. Exercise induces hippocampal BDNF through a PGC-1alpha/FNDC5 pathway. Cell Metab 18: 649-659, 2013.

432. Wu M and Gu Z. Screening of bioactive compounds from moutan cortex and their anti-inflammatory activities in rat synoviocytes. Evid Based Complement Alternat Med 6: 57-63, 2009.

433. Wu S, Zhou F, Zhang Z, and Xing D. Mitochondrial oxidative stress causes mitochondrial fragmentation via differential modulation of mitochondrial fission-fusion proteins. FEBS J 278: 941-954, 2011.

434. Wu Z, Puigserver P, Andersson U, Zhang C, Adelmant G, Mootha V, Troy A, Cinti S, Lowell B, Scarpulla RC, and Spiegelman BM. Mechanisms controlling mitochondrial biogenesis and respiration through the thermogenic coactivator PGC-1. Cell 98: 115-124, 1999.

435. Xia E-Q, Ai X-X, Zang S-Y, Guan T-T, Xu X-R, and Li H-B. Ultrasound-assisted extraction of phillyrin from Forsythia suspensa. Ultrason Sonochem 18: 549-552, 2011.

436. Xiao M, Yang H, Xu W, Ma S, Lin H, Zhu H, Liu L, Liu Y, Yang C, Xu Y, Zhao S, Ye D, Xiong Y, and Guan KL. Inhibition of alpha-KG-dependent histone and DNA demethylases by fumarate and succinate that are accumulated in mutations of $\mathrm{FH}$ and SDH tumor suppressors. Genes Dev 26: 1326-1338, 2012.

437. Xiao XQ, Wang R, and Tang XC. Huperzine A and tacrine attenuate $\beta$-amyloid peptide-induced oxidative injury. J Neurosci Res 61: 564-569, 2000.

438. Xie H, Guan J, Borrelli LA, Xu J, Serrano-Pozo A, and Bacskai BJ. Mitochondrial alterations near amyloid plaques in an Alzheimer's disease mouse model. J Neurosci 33: 17042-17051, 2013.

439. Xie J, Zhou Y, Dey L, Attele A, Wu J, Gu M, Polonsky K, and Yuan C-S. Ginseng berry reduces blood glucose and body weight in $\mathrm{db} / \mathrm{db}$ mice. Phytomedicine 9: 254-258, 2002.

440. Xie JT, Aung HH, Wu JA, Attele A, and Park J. Effects of American ginseng berry extract on blood glucose levels in ob/ob mice. Am J Chin Med 30: 187-194, 2002.

441. Xie S, Jin N, Gu J, Shi J, Sun J, Chu D, Zhang L, Dai CL, Gu JH, Gong CX, Iqbal K, and Liu F. O-GlcNAcylation of protein kinase A catalytic subunits enhances its activity: a mechanism linked to learning and memory deficits in Alzheimer's disease. Aging Cell 15: 455-464, 2016.

442. Xing ZG, Yu GD, Qin L, Jiang F, and Zhao WH. Effects and mechanism of lipoic acid on beta-amyloid-intoxicated C6 glioma cells. Genet Mol Res 14: 13880-13888, 2015.

443. Yamada $M$ and Naiki H. Cerebral amyloid angiopathy. Prog Mol Biol Transl Sci 107: 41-78, 2012.

444. Yang F, Zhang T, Zhang R, and Ito Y. Application of analytical and preparative high-speed counter-current chromatography for separation of alkaloids from Coptis chinensis Franch. J Chromatogr A 829: 137-141, 1998. 
445. Yao B, Ao Y, Zhang G, Liu M, Yao S, and Li D. Effect and mechanism of Gynostemma pentaphyllum Makino on learning and memory ability in dementia mice. Chin $J$ Public Health 33: 1708-1711, 2017.

446. Yao B, Yuan H, Huang X, and Zhao J. Effects of gypenosides on cell cycle-related protein expression and calcium homeostasis in hippocampal neurons of rats treated with $\beta$-amyloid protein fragment1-40. Chin J Pathophysiol 22: 1618-1622, 2006.

447. Yao J, Hamilton RT, Cadenas E, and Brinton RD. Decline in mitochondrial bioenergetics and shift to ketogenic profile in brain during reproductive senescence. Biochim Biophys Acta 1800: 1121-1126, 2010.

448. Yao J, Irwin RW, Zhao L, Nilsen J, Hamilton RT, and Brinton RD. Mitochondrial bioenergetic deficit precedes Alzheimer's pathology in female mouse model of Alzheimer's disease. Proc Natl Acad Sci U S A 106: 1467014675, 2009.

449. Yao J, Rettberg JR, Klosinski LP, Cadenas E, and Brinton RD. Shift in brain metabolism in late onset Alzheimer's disease: implications for biomarkers and therapeutic interventions. Mol Aspects Med 32: 247-257, 2011.

450. Yassine HN, Braskie MN, Mack WJ, Castor KJ, Fonteh AN, Schneider LS, Harrington MG, and Chui HC. Association of docosahexaenoic acid supplementation with Alzheimer disease stage in Apolipoprotein E epsilon4 carriers: a Review. JAMA Neurol 74: 339-347, 2017.

451. Yin F, Sancheti H, Patil I, and Cadenas E. Energy metabolism and inflammation in brain aging and Alzheimer's disease. Free Radic Biol Med 100: 108-122, 2016.

452. Yin J, Feng W, Yuan H, Yuan J, Wu Y, Liu X, Jin C, and Cheng Z. Association analysis of polymorphisms in STARD6 and near ECHDC3 in Alzheimer's disease patients carrying the APOE epsilon4 Allele. Neuropsychiatr Dis Treat 15: 213-218, 2019.

453. Young ML and Franklin JL. The mitochondria-targeted antioxidant MitoQ inhibits memory loss, neuropathology, and extends lifespan in aged 3xTg-AD mice. Mol Cell Neurosci 101: 103409, 2019.

454. Yuzwa SA, Shan X, Macauley MS, Clark T, Skorobogatko Y, Vosseller $\mathrm{K}$, and Vocadlo DJ. Increasing O-GlcNAc slows neurodegeneration and stabilizes tau against aggregation. Nat Chem Biol 8: 393-399, 2012.

455. Zandi PP, Anthony JC, Khachaturian AS, Stone SV, Gustafson D, Tschanz JT, Norton MC, Welsh-Bohmer KA, and Breitner JC. Reduced risk of Alzheimer disease in users of antioxidant vitamin supplements: the Cache County Study. Arch Neurol 61: 82-88, 2004.

456. Zeitlow K, Charlambous L, Ng I, Gagrani S, Mihovilovic M, Luo S, Rock DL, Saunders A, Roses AD, and Gottschalk WK. The biological foundation of the genetic association of TOMM40 with late-onset Alzheimer's disease. Biochim Biophys Acta Mol Basis Dis 1863: 2973 2986, 2017.

457. Zeng J, Chen L, Wang Z, Chen Q, Fan Z, Jiang H, Wu Y, Ren L, Chen J, Li T, and Song W. Marginal vitamin A deficiency facilitates Alzheimer's pathogenesis. Acta Neuropathol 133: 967-982, 2017.

458. Zhang H, Jia H, Liu J, Ao N, Yan B, Shen W, Wang X, Li $\mathrm{X}$, Luo C, and Liu J. Combined R- $\alpha$ lipoic acid and acetylL-carnitine exerts efficient preventative effects in a cellular model of Parkinson's disease. J Cell Mol Med 14: 215-225, 2010.
459. Zhang HY, Yan H, and Tang XC. Huperzine A enhances the level of secretory amyloid precursor protein and protein kinase $\mathrm{C}-\alpha$ in intracerebroventricular $\beta$-amyloid(1-40) infused rats and human embryonic kidney 293 Swedish mutant cells. Neurosci Lett 360: 21-24, 2004.

460. Zhang HY, Yan H, and Tang XC. Non-cholinergic effects of huperzine A: beyond inhibition of acetylcholinesterase. Cell Mol Neurobiol 28: 173-183, 2008.

461. Zhang J, Cao Q, Li S, Lu X, Zhao Y, Guan J-S, Chen J-C, Wu Q, and Chen G-Q. 3-Hydroxybutyrate methyl ester as a potential drug against Alzheimer's disease via mitochondria protection mechanism. Biomaterials 34: 75527562, 2013.

462. Zhang J, Zhang C-H, Li R-J, Lin X-L, Chen Y-D, Gao H-Q, and Shi S-L. Accuracy of urinary AD7c-NTP for diagnosing Alzheimer's disease: a systematic review and meta-analysis. J Alzheimers Dis 40: 153-159, 2014.

463. Zhang S, Wang Z, Cai F, Zhang M, Wu Y, Zhang J, and Song W. BACE1 cleavage site selection critical for amyloidogenesis and Alzheimer's pathogenesis. J Neurosci 37: 6915-6925, 2017.

464. Zhao B. Natural antioxidants protect neurons in Alzheimer's disease and Parkinson's disease. Neurochem Res 34: 630-638, 2009.

465. Zhao D, Sun Y, Tan Y, Zhang Z, Hou Z, Gao C, Feng P, Zhang $\mathrm{X}$, Yi W, and Gao F. Short-duration swimming exercise after myocardial infarction attenuates cardiac dysfunction and regulates mitochondrial quality control in aged mice. Oxid Med Cell Longev 2018: 4079041, 2018.

466. Zhao L, Feng Z, Yang X, and Liu J. The regulatory roles of O-GlcNAcylation in mitochondrial homeostasis and metabolic syndrome. Free Radic Res 50: 1080-1088, 2016.

467. Zhao L, Feng Z, Zou X, Cao K, Xu J, and Liu J. Aging leads to elevation of O-GlcNAcylation and disruption of mitochondrial homeostasis in retina. Oxid Med Cell Longev 2014: 425705, 2014.

468. Zhao N, Liu CC, Van Ingelgom AJ, Martens YA, Linares C, Knight JA, Painter MM, Sullivan PM, and Bu G. Apolipoprotein E4 impairs neuronal insulin signaling by trapping insulin receptor in the endosomes. Neuron 96 : 115-129.e5, 2017.

469. Zheng A, Li H, Xu J, Cao K, Li H, Pu W, Yang Z, Peng Y, Long J, and Liu J. Hydroxytyrosol improves mitochondrial function and reduces oxidative stress in the brain of $\mathrm{db} / \mathrm{db}$ mice: role of AMP-activated protein kinase activation. Br J Nutr 113: 1667-1676, 2015.

470. Zheng Y, Sun T, Wang M, Li R, Yao B, and Tan H. The influence of Steven leafs on the cell's model of AD induced by A $\beta 1-40$. Chin J Integr Med Cardio/Cerebrovasc Dis 10: 201-204, 2012.

471. Zhou J, Zhou L, Hou D, Tang J, Sun J, and Bondy SC. Paeonol increases levels of cortical cytochrome oxidase and vascular actin and improves behavior in a rat model of Alzheimer's disease. Brain Res 1388: 141-147, 2011.

472. Zhou L, Brouwers N, Benilova I, Vandersteen A, Mercken M, Van Laere K, Van Damme P, Demedts D, Van Leuven $\mathrm{F}$, and Sleegers K. Amyloid precursor protein mutation E682K at the alternative $\beta$-secretase cleavage $\beta^{\prime}$-site increases A $\beta$ generation. EMBO Mol Med 3: 291-302, 2011.

473. Zhou R, Yang G, Guo X, Zhou Q, Lei J, and Shi Y. Recognition of the amyloid precursor protein by human gamma-secretase. Science 363: pii: eaaw0930, 2019. 
474. Zhou R, Yazdi AS, Menu P, and Tschopp J. A role for mitochondria in NLRP3 inflammasome activation. Nature 469: 221-225, 2011.

475. Zilberter M, Ivanov A, Ziyatdinova S, Mukhtarov M, Malkov A, Alpár A, Tortoriello G, Botting CH, Fülöp L, and Osypov AA. Dietary energy substrates reverse early neuronal hyperactivity in a mouse model of Alzheimer's disease. J Neurochem 125: 157-171, 2013.

476. Zou X, Feng Z, Li Y, Wang Y, Wertz K, Weber P, Fu Y, and Liu J. Stimulation of GSH synthesis to prevent oxidative stress-induced apoptosis by hydroxytyrosol in human retinal pigment epithelial cells: activation of Nrf2 and JNK-p62/SQSTM1 pathways. J Nutr Biochem 23: 9941006, 2012.

Address correspondence to: Dr. Jiankang Liu Center for Mitochondrial Biology \& Medicine The Key Laboratory of Biomedical Information Engineering of Ministry of Education School of Life Science and Technology Frontier Institute of Science and Technology $X i$ 'an Jiaotong University 28 West Xianning Road Xi'an 710049 China

E-mail: j.liu@mail.xjtu.edu.cn

Dr. Jiangang Long Center for Mitochondrial Biology \& Medicine The Key Laboratory of Biomedical Information Engineering of Ministry of Education

School of Life Science and Technology Frontier Institute of Science and Technology $X i$ 'an Jiaotong University 28 West Xianning Road $X i$ 'an 710049

China

E-mail: jglong@mail.xjtu.edu.cn

Date of first submission to ARS Central, June 4, 2019; date of final revised submission, February 9, 2020; date of acceptance, February 10, 2020.

Abbreviations Used
$2-\mathrm{HG}=2$-hydroxyglutarate
$5 \mathrm{hmC}=5$-hydroxymethylcytosine
$5 \mathrm{mC}=5$-methylcytosine
$\beta-\mathrm{HB}=\beta$-hydroxybutyrate
$\mathrm{A} \beta=$ beta amyloid
$\mathrm{ABAD}=\mathrm{A} \beta$-binding alcohol dehydrogenase
$\mathrm{ABCA} 7=\mathrm{ATP}$ binding cassette subfamily $\mathrm{A}$
$\quad$ member 7
$\mathrm{AD}=$ Alzheimer's disease
$\mathrm{ADAS}-\mathrm{cog}=$ Alzheimer's Disease Assessment Scale-
$\quad$ Cognitive Subscale
$\mathrm{ADH}=$ alcohol dehydrogenase
$\mathrm{ADL}=$ activities of daily living
$\mathrm{ADP}=$ adenosine diphosphate
$\mathrm{AGE}=$ advanced glycation end-product

$\mathrm{AKT}=$ protein kinase $\mathrm{B}$ (also known as $\mathrm{PKB}$ )

ALCAR $=$ acetyl-L-carnitine

AMPK $=$ adenosine monophosphate-activated protein kinase

$\mathrm{APE} 1=\mathrm{AP}$ endonuclease 1

$\mathrm{ApoE}=$ apolipoprotein $\mathrm{E}$

$\mathrm{APOP}=$ apoptosis

$\mathrm{APP}=$ amyloid beta precursor protein

$\mathrm{ARE}=$ antioxidant response element

$\mathrm{ATP}=$ adenosine triphosphate

$\mathrm{BACE} 1=\beta$-site APP cleaving enzyme 1

$\mathrm{BBB}=$ blood-brain barrier

$\mathrm{BDNF}=$ brain-derived neurotrophic factor

$\mathrm{BHD} 1=\beta$-hydroxybutyrate dehydrogenase 1

$\mathrm{BIN} 1=$ bridging integrator 1

$\mathrm{CaMKII}=\mathrm{Ca}^{2+} /$ calmodulin-dependent protein kinase II

$\mathrm{CCH}=$ coral calcium hydride

CDK5 $=$ cyclin-dependent kinase 5

cfDNA $=$ cell-free DNA

$\mathrm{CLU}=$ clusterin

$\mathrm{CNS}=$ central nervous system

CoQ10 = coenzyme Q10

$\mathrm{COX}=$ cytochrome $\mathrm{c}$ oxidase

$\mathrm{CR} 1=$ complement $\mathrm{C} 3 \mathrm{~b} / \mathrm{C} 4 \mathrm{~b}$ receptor 1

$\mathrm{CsA}=$ cyclosporine $\mathrm{A}$

$\mathrm{CSF}=$ cerebrospinal fluid

$\mathrm{CTS}=$ cryptotanshinone

CypD $=$ cyclophilin D

DHA $=$ docosahexaenoic acid

Drp1 = dynamin-related protein 1

ECHDC3 = enoyl CoA-hydratase domain containing 3

ECHS1 = enoyl-CoA hydratase

$\mathrm{EGCG}=$ epigallocatechin-3-gallate

$\mathrm{EPA}=$ eicosapentaenoic acid

$\mathrm{ER}=$ endoplasmic reticulum

ERK1/2 = extracellular signal regulated kinase $1 / 2$

$\mathrm{FAD}=$ familial $\mathrm{AD}$

FDG-PET = fluoro-2-deoxy-D-glucose positron emission tomography

Fis $1=$ mitochondrial fission 1

FoxO3a $=$ forkhead box O3a

$\mathrm{GK}=$ Goto-Kakizaki

GLP-1 = glucagon-like peptide 1

GLUTs $=$ glucose transporters

GSK3 = glycogen synthesis kinase 3

GWAS $=$ genome-wide association studies $\mathrm{H}_{2}=$ hydrogen

HDACs $=$ histone deacetylases

$\mathrm{HFD}=$ high-fat diet

HMG-CoA $=\beta$-hydroxy- $\beta$-methylglutaryl-CoA

$\mathrm{HSL}=$ hormone-sensitive lipase

HT $=$ hydroxytyrosol

HT-ac $=$ hydroxytyrosol acetate

$\mathrm{IDE}=$ insulin degrading enzyme

$\mathrm{IF}=$ intermittent fasting

IGF-I = insulin-like growth factor I

$\mathrm{INF}=$ inflammation

$\mathrm{IR}=$ insulin receptor

$\mathrm{JNK}=\mathrm{C}$-Jun NH2-terminal kinase

$\mathrm{KGDH}=\alpha$-ketoglutarate dehydrogenase complex

LA $=\alpha$-lipoic acid

LRP2 $=$ lipoprotein receptor-related protein 2

$\mathrm{MAPK}=$ mitogen-activated protein kinase 

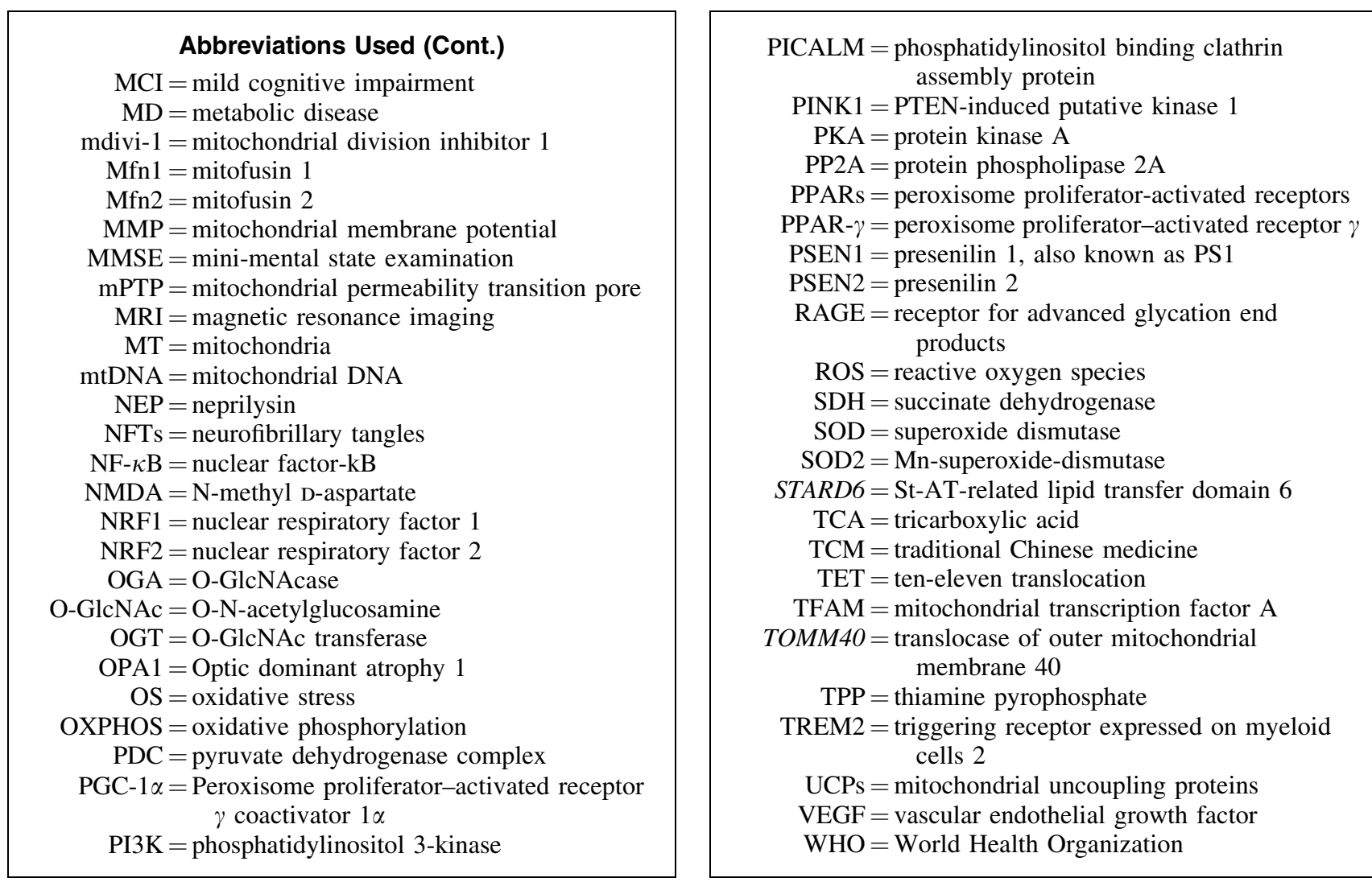